\title{
Effects of experimental febrile seizures on the developing brain
}

Citation for published version (APA):

Lemmens, E. M. P. (2008). Effects of experimental febrile seizures on the developing brain. [Doctoral Thesis, Maastricht University]. Universiteit Maastricht. https://doi.org/10.26481/dis.20081126el

Document status and date:

Published: 01/01/2008

DOI:

$10.26481 /$ dis.20081126el

Document Version:

Publisher's PDF, also known as Version of record

\section{Please check the document version of this publication:}

- A submitted manuscript is the version of the article upon submission and before peer-review. There can be important differences between the submitted version and the official published version of record.

People interested in the research are advised to contact the author for the final version of the publication, or visit the DOI to the publisher's website.

- The final author version and the galley proof are versions of the publication after peer review.

- The final published version features the final layout of the paper including the volume, issue and page numbers.

Link to publication

\footnotetext{
General rights rights.

- You may freely distribute the URL identifying the publication in the public portal. please follow below link for the End User Agreement:

www.umlib.nl/taverne-license

Take down policy

If you believe that this document breaches copyright please contact us at:

repository@maastrichtuniversity.nl

providing details and we will investigate your claim.
}

Copyright and moral rights for the publications made accessible in the public portal are retained by the authors and/or other copyright owners and it is a condition of accessing publications that users recognise and abide by the legal requirements associated with these

- Users may download and print one copy of any publication from the public portal for the purpose of private study or research.

- You may not further distribute the material or use it for any profit-making activity or commercial gain

If the publication is distributed under the terms of Article $25 \mathrm{fa}$ of the Dutch Copyright Act, indicated by the "Taverne" license above, 


\section{Effects of Experimental Febrile Seizures on the Developing Brain}




\section{Cover}

The cover illustration depicts a newborn hippocampal neuron, fluorescently labeled with bromodeoxyuridine (BrdU) in red and the glutamate transporter $\mathrm{EAACl}$ in green.

(c) 2008 Evi Lemmens

ISBN: 978-90-9023582-0

Production: PrintPartners Ipskamp, The Netherlands 


\title{
Effects of Experimental Febrile Seizures on the Developing Brain
}

\author{
PROEFSCHRIFT \\ ter verkrijging van de graad van doctor \\ aan de Universiteit M aastricht \\ op gezag van de Rector Magnificus, Prof. M r. G.P.M .F. M ols \\ volgens het besluit van het College van Decanen \\ in het openbaar te verdedigen \\ op woensdag 26 november 2008 om 14.00 uur \\ door
}

Evi Maria Petra Lemmens 


\section{Promotores}

Prof. Dr. H.W.M. Steinbusch

Prof. Dr. E.A.M. Beuls

\section{Copromotor}

Dr. G. Hoogland

\section{Beoordelingscommissie}

Prof. Dr. J.S.H. Vles (voorzitter)

Prof. Dr. A.P. Aldenkamp

Prof. Dr. P.A.J.M. Boon (Universiteit Gent)

Prof. Dr. M. Offringa (Academisch M edisch Centrum Amsterdam)

Dr. S. Robben 
"Science is a critical activity. We examine our hypotheses critically. We criticize them so that we can find errors, In the hope of eliminating the errors, And thus getting closer to the truth."

Karl Popper 


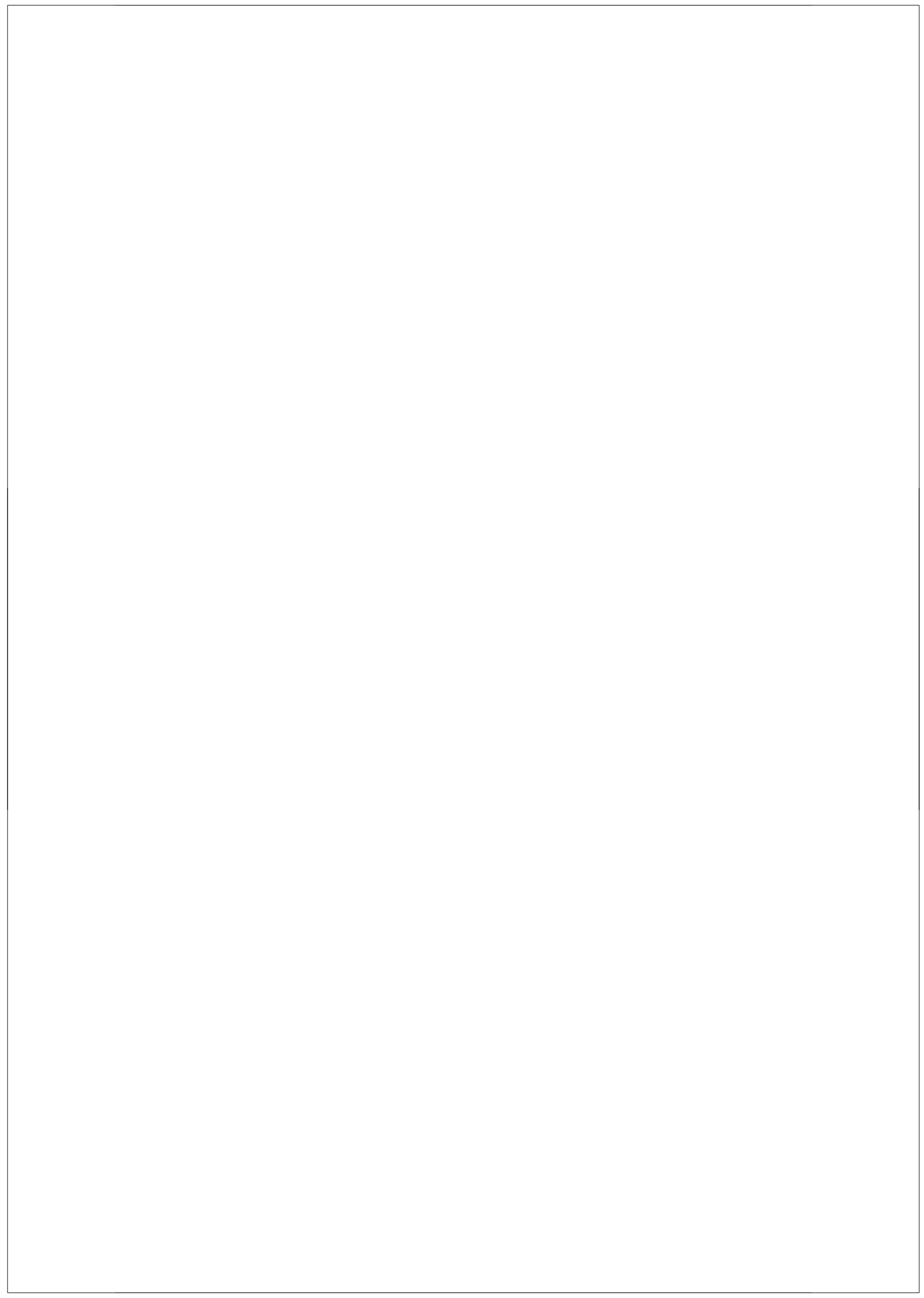




\section{Contents}

List of abbreviations $\quad 8$

$\begin{array}{lll}\text { Chapter } 1 \text { Introduction } & 11\end{array}$

Chapter 2 Gender differences in febrile seizure-induced proliferation 29 and survival in the rat dentate gyrus

Chapter 3 Cytogenesis in the dentate gyrus after neonatal hyperthermia- 57 induced seizures: What becomes of surviving cells?

Chapter 4 Short- and long-term limbic abnormalities after experimental $\quad 77$ febrile seizures

Chapter 5 Experimental febrile seizures do not cause long-lasting changes in glutamate transporter-mediated neurophysiology

Chapter 6 Long-term behavioral outcome after early-life hyperthermiainduced seizures

Chapter 7 General discussion

Summary

Samenvatting

Dankwoord

List of publications

Curriculum Vitae 


\section{List of abbreviations}

\begin{tabular}{|c|c|}
\hline$A$ & amygdala \\
\hline ADC & apparent diffusion coefficient \\
\hline AED & antiepileptic drug \\
\hline BBB & blood-brain barrier \\
\hline BrdU & bromodeoxyuridine \\
\hline $\mathrm{CC}$ & corpus callosum \\
\hline CHESS & chemical shift selective suppression \\
\hline Cho & choline \\
\hline $\mathrm{Cr}$ & creatine \\
\hline CREB & cAMP response-element binding \\
\hline CRT & choice reaction time \\
\hline DG & dentate gyrus \\
\hline DGC & dentate granule cell \\
\hline DGL & dentate granule cell layer \\
\hline DTI & diffusion tensor imaging \\
\hline EAAT & excitatory amino acid transporter \\
\hline EEG & electroencephalography \\
\hline FA & fractional anisotropy \\
\hline FS & febrile seizures \\
\hline GABA & gamma-aminobutyric acid \\
\hline GABAR & GABA receptor \\
\hline GAD & glutamic acid decarboxylase \\
\hline GFAP & glial fibrillary acidic protein (astrocyte marker) \\
\hline Glu & glutamate \\
\hline $\mathrm{HC}$ & hippocampus \\
\hline $\mathrm{HCN}$ & hyperpolarization-activated cyclic nucleotide-gated \\
\hline HE & hematoxylin-eosin \\
\hline${ }^{1} \mathrm{H}-\mathrm{MRS}$ & proton magnetic resonance spectroscopy \\
\hline $\mathrm{HT}$ & hyperthermia \\
\hline HS & hippocampal sclerosis \\
\hline I & inositol \\
\hline
\end{tabular}


KA

LTLE

MR

MRI

MSME

MT

MTLE

MTS

MWM

NAA

NDS

NeuN

NT

$\mathrm{PC}$

PN

PR

PRESS

$\mathrm{RC}$

$\mathrm{ROI}$

RT

SE

Tau

TBOA

TBS

TE

$\mathrm{TH}$

TL

TLE

TR

VGLUT kainic acid

lateral temporal lobe epilepsy

magnetic resonance

magnetic resonance imaging

multi slice multi spin-echo

motor time

mesial temporal lobe epilepsy

mesial temporal sclerosis

morris water maze

$\mathrm{N}$-acetyl-aspartate

normal donkey serum

neuronal nuclei (neuronal cell marker)

normothermia

piriform cortex

postnatal day

premature response

point-resolved spectroscopy

retrosplenial cortex

region of interest

reaction time

status epilepticus

taurine

dl-threo- $\beta$-benzyloxyaspartic acid

tris-buffered saline

echo times

thalamus

tomato lectin (microglia marker)

temporal lobe epilepsy

repetition time

vesicular glutamate transporter 


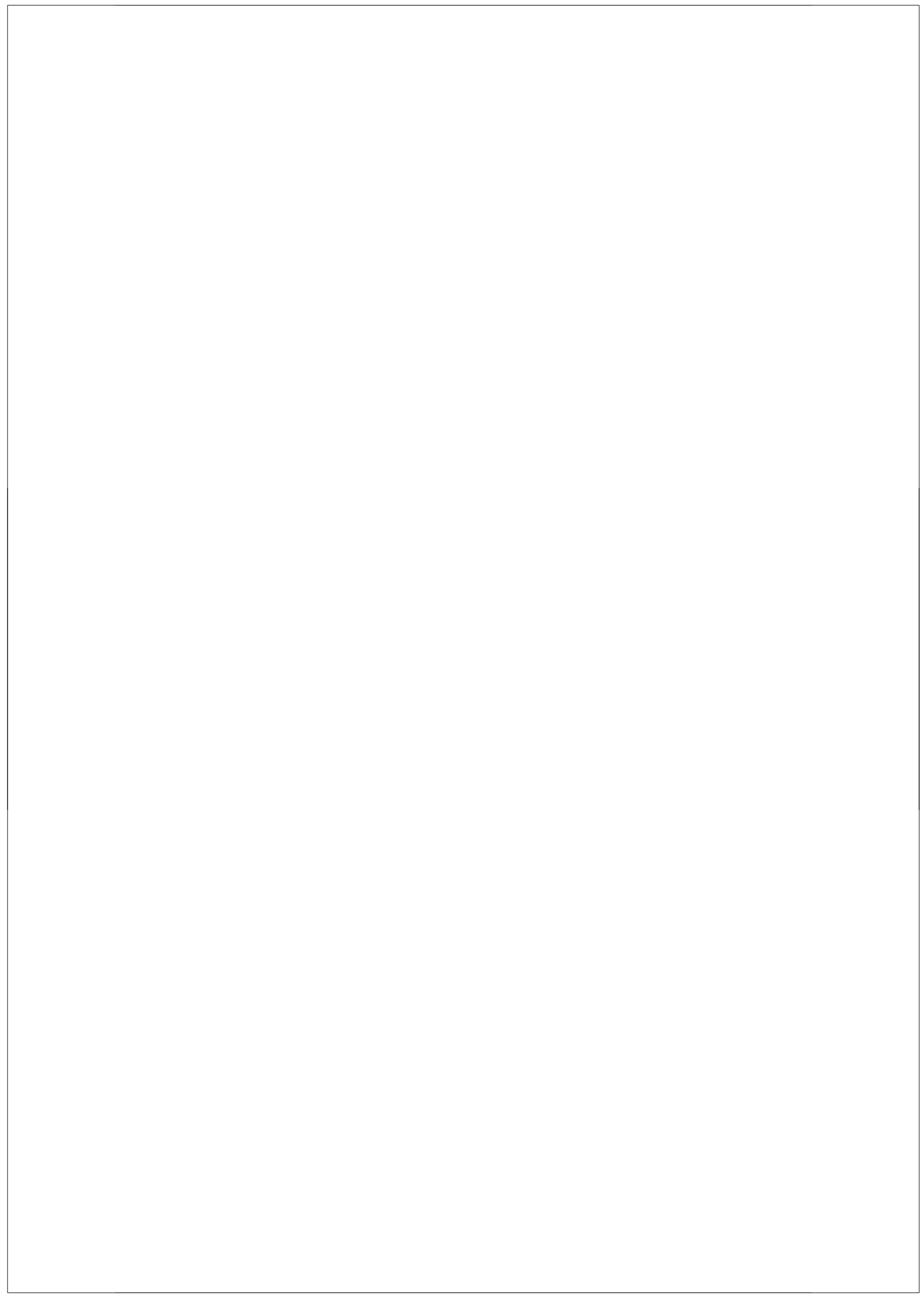




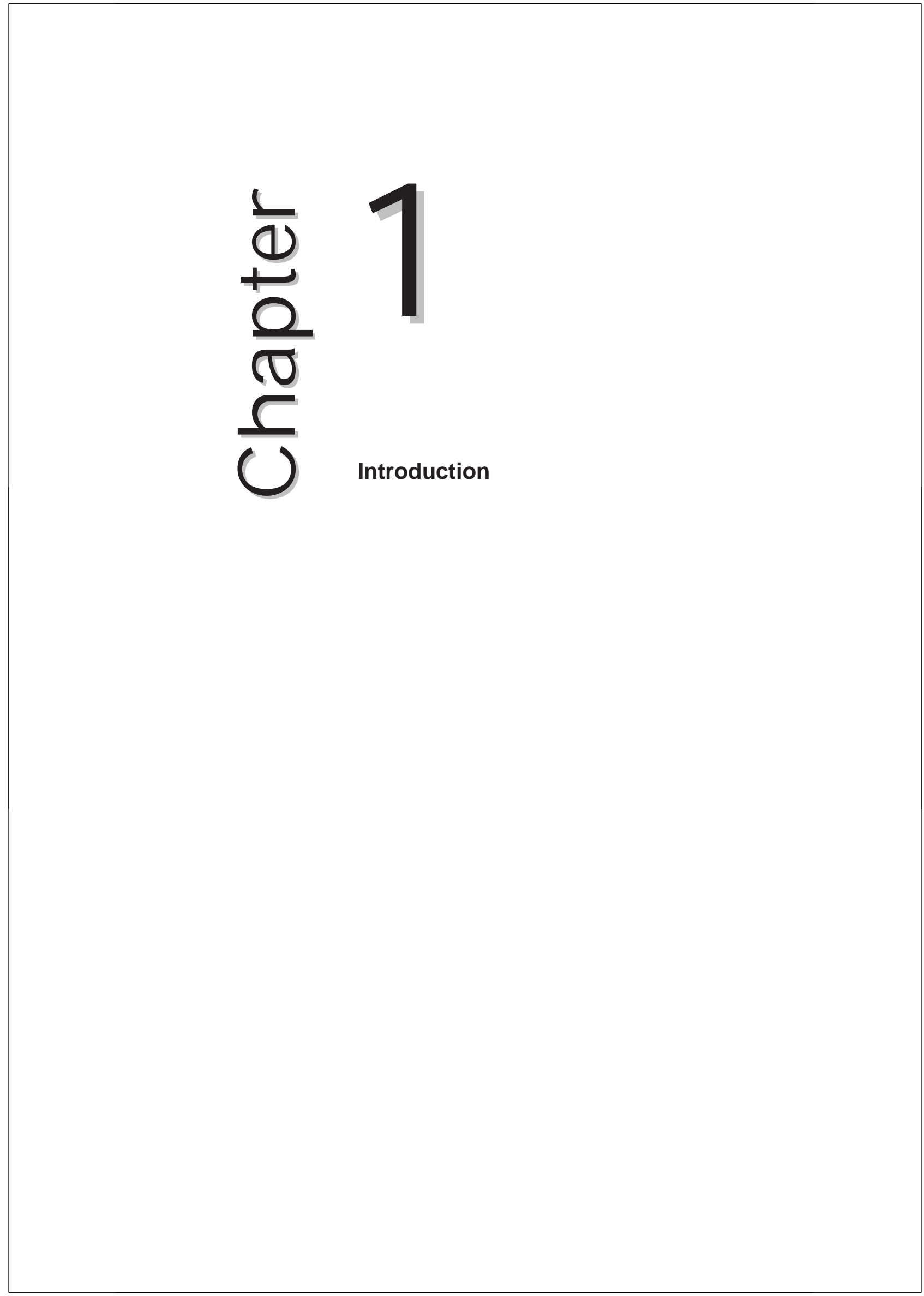




\section{Epilepsy}

Epilepsy is one of the most common chronic neurological disorders that affects about $1 \%$ of the world's population. Hippocrates was the first to recognize epilepsy as a disease of the brain. Before, it was believed to be the work of supernatural forces, and seizing people were thought to be possessed. Today, epilepsy is defined as a syndrome, characterized by recurrent unprovoked seizures, which are due to occasional, excessive or abnormal, synchronous firing of neurons, and detected by clinical observation and electroencephalographic (EEG) recording of brain activity [1]. In general, epilepsy is recognized as a state of chronic hyperexcitability, resulting from an imbalance between excitation and inhibition.

Seizures are mainly classified according to the site of origin. In that respect, one speaks of 'partial seizures' when only part of the brain is involved and confined to one hemisphere, or 'generalized seizures' if both hemispheres participate in seizure activity. Partial seizures can be further subdivided into simple, usually short without loss of consciousness, and complex partial seizures, which last longer and in which consciousness is impaired. Furthermore, partial seizures can sometimes spread throughout the brain, and are accordingly called 'secondarily generalized seizures' [2]. Any seizure that does not fit within one of these categories, is termed 'unclassified'.

\section{Temporal lobe epilepsy}

Temporal lobe epilepsy (TLE) is one of the most common epilepsy syndrome, and patients suffer from partial seizures arising in structures of the temporal lobe. Two types of TLE are known: lateral TLE (LTLE) and mesial TLE (MTLE). In LTLE, seizures begin as simple partial seizures with a focus in the temporal neocortex, characterized by auditory features, that may progress to complex partial seizures when they spread to structures of the mesial temporal lobe. MTLE is much more common than LTLE and seizures arising in the mesial 
structures of the temporal lobe (e.g. hippocampus, amygdala) are stereotyped and accompanied by epigastric sensations, nausea, autonomic signs, arrest of respiration, etc. The usual etiologies for partial seizures include structural abnormalities (e.g. developmental defects, tumors), traumatic brain injury, and infections. Although genetic defects are most relevant to generalized seizures, they can also be involved in predisposition to partial seizures [3]. The treatment of choice for epilepsy patients is the administration of antiepileptic drugs (AEDs). Unfortunately, patients with the MTLE syndrome are often 'refractory', meaning that they do not respond to AED treatment [4,5]. They typically suffered from seizures (mostly complex) during childhood caused by an initial precipitating event. After a silent period ranging from 2 to 15 years, referred to as 'epileptogenesis', these children develop spontaneous partial seizures [6-8].

Hippocampal sclerosis (HS), also called Ammon's Horn sclerosis or mesial temporal sclerosis (MTS), is the major pathological substrate found in about $65 \%$ of the refractory MTLE patients. The major histological hallmarks of HS were first described by Sommer in 1880 and a grading system, ranging from mild (grade 1) to very severe (grade 5) HS was proposed by Wyler and colleagues more than a century later [9]. Hippocampal neuron loss, which is most prominent in the pyramidal CA1 and CA3 layer and in the hilus with relatively sparing of the CA2 and dentate granule cell layer (DGL), is found, accompanied by reactive gliosis, mossy fiber sprouting, and granule cell dispersion, with concurrent abnormalities in other temporal lobe structures such as the amygdala [10-14]. Mossy fiber sprouting is thought to arise as a consequence of the loss of the appropriate target of the mossy fibers. Normally, mossy fibers from dentate granule cells (DGCs) innervate hilar mossy cells and CA3 pyramidal cells. Due to the loss of mossy cells and CA3 neurons, mossy fibers sprout and synapse back mainly onto the excitatory DGCs [15]. Neurogenesis, or the birth of new neurons, has been found to persist in the adult brain, and it has been shown that MTLE patients have an increased number of progenitor cells [13]. Granule cell dispersion, or widening of the DGL, and often accompanied by the appearance of ectopic granule cells in the hilus, 
has been thought to be related to a disturbance of neurogenesis and migration of newborn cells. In this respect, a deficiency in reelin signaling, a protein secreted by Cajal-Retzius cells and highly important for the migration of neurons during development, has been detected in MTLE patients [16]. In vivo, magnetic resonance imaging (MRI) is able to provide reliable information about the possible presence of hippocampal sclerosis. The most prominent MR finding of HS is atrophy or volume loss of the ipsilateral hippocampus (i.e. the hippocampus on the side of the seizure focus) due to neuronal cell loss, which is often accompanied by increased T2 signal intensity reflecting gliosis. Besides the hippocampus, also the amygdala, the temporal cortex, and the thalamus are among the structures that can show increased signal intensity, volume loss, or both [17]. Controversy remains whether HS is a cause or a consequence of recurrent seizures in TLE. However, the existence of granule cell dispersion and increased neurogenesis for example, and the presence of high numbers of Cajal-Retzius cells that are normally almost undetectable in adulthood, indicate a disturbance in neurodevelopmental processes. It is therefore likely to assume that the occurrence of HS is related to an early precipitating event, such as early-life seizures. 


\section{The developing brain - A state of hyperexcitability}

FS occur during a critical time window of brain development, hence their agespecificity. It so happens that the immature brain is highly sensitive to seizure activity, i.e. the threshold for developing a seizure in the immature brain is much lower than in the adult brain. Several mechanisms that contribute to normal brain development, being slow maturation of inhibitory neurotransmission and overexpression of excitatory neurotransmission, may also account for this state of hyperexcitability [18].

Studies have shown that the expression of ion channels, and neurotransmitter receptors and transporters, is developmentally regulated (reviewed in [19]). For instance, the immature brain expresses only low levels of the $\mathrm{NA}^{+} / \mathrm{K}^{+}$-ATPase, leading to an imbalanced $\mathrm{K}^{+}$-gradient and changes in the membrane potential; also, the chloride gradient is reversed compared to the adult brain. Before the third postnatal week, chloride channels and transporters are not functioning properly, resulting in a high concentration of chloride inside the cell instead of outside the cell. So when GABA receptors (GABARs) become activated, a net efflux of chloride causes depolarization. Not only GABAergic neurotransmission, but also glutamate receptor and transporter expression change during maturation. For example, glutamatergic NMDA and AMPA receptor density peaks at the end of the first and second postnatal week, respectively. In addition, a different subunit composition of these receptors during development further enhances excitation: NMDA receptors are composed mainly of NR2D subunits instead of NR2B subunits, which renders them more permeable to $\mathrm{Ca}^{+2}$ and less sensitive to blockage by magnesium; and AMPA receptors are also more permeable to $\mathrm{Ca}^{+2}$ because of a low expression of the GluR2 subunit. Glutamate transporter expression, which in the adult brain reduces excitatory neurotransmission and thereby also vulnerability to cell death, is only gradually increased in the immature brain. To date, five subtypes of excitatory amino acid (glutamate) transporters (EAATs) have been cloned from animal and human 
tissue, named GLAST/EAAT1, GLT-1/EAAT2, EAAC1/EAAT3, EAAT4 and EAAT5. Histological studies have localized EAAT1 and 2 primarily in astrocytes and EAAT3 and 4 to neurons. EAAT5 is exclusively expressed in the retina and will not be discussed further. Studies in animals have indicated that the expression of each glutamate transporter subtype is developmentally regulated. Prenatally, GLT-1 and EAAC1 are mainly present, whereas GLAST and EAAT4 expression is very low. The most important changes occur postnatally with increases in the expression of all EAATs; GLT-1 is mainly expressed in astrocytes after postnatal day (PN) 10, and the expression of EAAC1 is strong from PN10 onwards. By PN24, each EAAT had reached the adult levels of expression. Focusing on the hippocampus, GLT-1 and EAAC1 are more enriched than GLAST and EAAT4 during postnatal development. At PN10, EAAC1 immunoreactivity is found to be very high in the CA1-3 area and the DGL but the levels decrease in adulthood [20].

Although all of these processes lead to a decreased seizure threshold, the excitatory actions of GABA, the $\mathrm{Ca}^{+2}$ permeability of the NMDA and AMPA receptors etc., during this stage of maturation, have been shown to be necessary for processes such as neurogenesis, migration and differentiation, and synaptogenesis, thus for normal brain development and plasticity [21]. The state of hyperexcitability is developmentally regulated and normally only transient. However, it may become long-lasting, persisting into adulthood, due to an early-life insult such as FS or other factors as genetic predisposition. FS may thereby decrease threshold for further unprovoked seizures, thus increasing the probability of developing epilepsy. 


\section{Febrile Seizures}

Febrile seizures (FS) are the most common type of early-life seizures. They occur in $2-5 \%$ of children between 6 months and 5 years of age, with a peak incidence at 18 months, in association with a fever but without evidence of intracranial infection $[22,23]$. FS are typically divided into two types, simple and complex. Most children suffer from simple FS which usually resolve spontaneously within 15 minutes and are mainly generalized tonic-clonic seizures, while others have complex FS that have a focal onset and last longer than 15 minutes [24]. Some studies have indicated that FS are slightly more common in boys than in girls $[25,26]$. However, large epidemiological studies did not verify this difference [22]. A few studies have examined possible risk factors of experiencing a FS and the most consistent factor found in these studies is a family history of FS, with the risk of developing FS being about $10 \%$ (i.e. 2-3 times higher than in the general population) [23]. Other risk factors include developmental delay, neonatal hospital care mostly in case of prematurity, and attendance at day care that is probably due to the increased risk of infections. When two or more of these risk factors are present, the risk of having a first FS was found to be substantially increased to about $30 \%$.

\section{Long-term outcome after FS}

FS are considered to be mostly benign, i.e. they are associated with low mortality and morbidity, and normal neurological, behavioral, intellectual and cognitive outcome [26-28]. However, some children with FS have been shown to suffer from hyperactivity, sleep problems, and anxiety [28-30]. The risk of developing unprovoked seizures and epilepsy after a simple FS is not much different from the general population [31]. On the other hand, complex FS, a family history of FS, preexisting neurodevelopmental abnormalities, and especially a combination of these factors, have been associated with an 
increased risk, ranging from a 2 to 3 -fold higher risk when only one factor is present to a 14-fold increased risk when all three factors are present [26,31-34].

One of the most controversial issues in epilepsy research is the relationship between FS and the subsequent development of TLE. Retrospective studies have demonstrated a significant link between a history of FS in childhood and the development of MTS-associated TLE, with a more than 10 times higher risk in patients that experienced FS [35-38]. On the other hand, prospective population-based studies failed to support a causal role of FS in the development of TLE. In particular, it was shown that treatment of FS does not interfere with the development of epilepsy [39]. However, most of these studies did not focus specifically on TLE but they studied the development of epilepsy in general. Therefore, the occurrence of TLE after FS may have been underestimated. Some epidemiological studies (e.g. [34]) did study the association between FS and TLE in particular, but could not find a significant relationship in the TLE patient group as a whole. In contrast, they did find a strong association between FS and the development of intractable TLE. As mentioned previously, several characteristics of HS, often found in these refractory patients, point towards the occurrence of an early-life event such as FS.

A severe limitation of prospective studies of the long-term outcome of FS is the duration of the follow-up period. A latency period of about 10 years exists between the occurrence of FS and the development of TLE, so the follow-up period should be long. This is quite difficult and expensive, and the reason why these studies are rare. Therefore, and because of the obvious ethical reasons of studies in humans, animal models of FS are proven to be highly valuable.

\section{Animal models of FS}

For more than 25 years animal models for FS have been developed. Most of these animal models aimed at mimicking fever by increasing the body temperature with exposure to a heat source such as a microwave [40-42], 
infrared light $[43,44]$, warm water $[45,46]$ or air $[47,48]$. It is important to note that animal models are merely an estimate of the clinical situation. This is especially true for multifactorial diseases such as epilepsy. A well-designed animal model should thus include at least the key features of the condition studied. In the case of a model of FS, several characteristics need to be considered. First of all, FS occur during a specific time window and susceptibility to FS decreases with age. In this respect, it was shown that susceptibility to HT-induced FS declines at the end of the second postnatal week in the rat [41]. The advantage of the model developed by Baram and colleagues is that they use 10-day-old rats which corresponds best to a stage of brain development comparable to that at which children are most susceptible for FS. Others have mostly used younger (2-10 days [44], or 3 days [42]) or older animals (16 days [43], 22 days [45], 25-30 days [40], young adults [46]). Secondly, threshold temperatures, required to induce a seizure, are also related to the maturational stage of the brain and should be in the physiological range. Most of the animal models use threshold temperatures of about $40^{\circ} \mathrm{C}$, however some use higher temperatures [45] and the microwave models [40,41] only notice a slight increase in temperature needed to induce FS, making them less suitable to model FS. Thirdly, mortality and morbidity should be low, comparable to the 'benign' nature of FS in humans, and because it makes the model highly suitable for long-term follow-up studies. All of these three criteria are met in the HT model developed by Baram and colleagues $[47,49]$ so for the experiments published in this thesis, we used this model to study the long-term effects of FS.

\section{Consequences of hyperthermia-induced FS in animals}

Using the animal model of HT-induced FS, several structural, molecular and functional changes induced by FS have been already found. While neuronal injury was detected, no evidence of neuronal cell loss has been found, nor changes in neurogenesis until 4 weeks after FS. Mossy fiber sprouting, found in 
tissue from MTS-TLE patients, was only moderately increased and T2 signal intensity, measured using MRI, was increased for at least one month. Molecular changes included a transient increase in the level of interleukin-1 $\beta$, neuropeptide $Y$, and c-fos, and a transient decreased expression of the GluR2 subunit of the AMPA glutamate receptors. A long-term decreased phosphorylation of CAMP response-element binding (CREB) and Erk, as well as a decreased hyperpolarization-activated cyclic-nucleotide-gated channel (HCN) 1 expression was found, the latter leading to an altered current generated by these channels. At the functional level, HT-induced FS were associated with a decreased GABAergic inhibition, a decreased threshold for kainic acid-induced seizures, and ultimately spontaneous nocturnal seizures in $35 \%$ of the animals that experienced FS (reviewed in [50]).

Though our knowledge about the effects of FS in the developing brain is increasing, there are still unresolved issues that require further investigation. Especially genetic susceptibility to FS and its contribution to the development of TLE is unclear. More and better information on the mechanisms of FS-induced epileptogenesis and the long-term consequences of this process is needed. Ultimately, answers on all these questions will improve treatment and care of FS patients, and perhaps interfere with the development of TLE.

\section{Hypotheses for the link between FS and TLE: First vs. Second Hit}

Still unresolved, but very intriguing, is the question whether FS are the cause of MTS or an epiphenomenon. In the first case, the so-called 'first hit' hypothesis, FS directly damage the hippocampus, thereby leading to processes involved in epileptogenesis and subsequent development of epilepsy. Alternatively, FS are might be the expression of prior damage to the hippocampus by an initial insult or genetic predisposition, referred to as the 'second hit' hypothesis. Another possibility is that a 'second hit', occurring during epileptogenesis after FS, might be necessary for the switch from hyperexcitability to the development of spontaneous seizures and epilepsy. Both hypotheses are represented in the 
following scheme (line-arrow: first hit hypothesis; dashed line-arrow and blockarrow: second hit hypothesis):

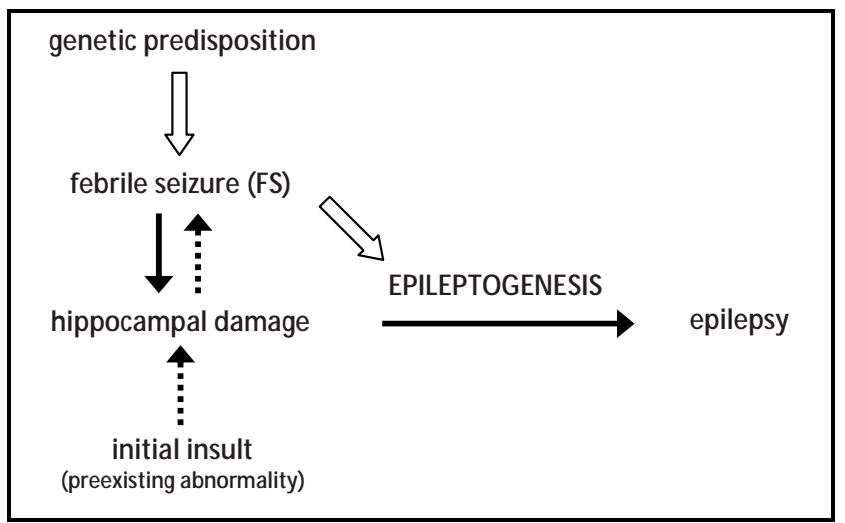

For long it was thought that FS injure the hippocampus and result in TLE. Recently published data from the clinical and laboratory however, are more in favor of the 'second hit' hypothesis. The use of magnetic resonance imaging (MRI) has contributed to this mentality switch, because MRI has the ability to detect HS when it is still subclinical, many years before TLE emerges. For example, prospective MRI studies performed after FS in children, found that complex lateralized or focal FS were associated with a more severe grade of HS than generalized FS. One possible mechanism is the occurrence of a preexisting abnormality in the hemisphere of the seizure focus [51-53]. Others showed that in families with FS, subtle hippocampal abnormalities are present even in asymptomatic individuals. This suggests preexisting damage resulting in FS and/or MTLE [54]. Furthermore, rats with a preexisting pathology were more susceptible to FS induction than rats that experienced normal brain development, and developed more severe damage afterwards $[55,56]$. Animal models not only permit a more controlled and standardized experimental design, they also need a shorter follow up period, and have thus been proven to be highly valuable in helping clinical studies to unravel the link between FS and TLE. 


\section{Aim \& Outline of this thesis}

The aim of this thesis was to test the hypothesis that hyperexcitability induced by early-life FS is related to disruption of developmental brain processes, with a focus on changes in glutamatergic neurotransmission, and their role in the longterm outcome of FS. For this purpose, several experiments were performed using an animal model of HT-induced FS, and the results are described in the following chapters.

Neurogenesis is important for normal brain development, is shown in other animal models of epilepsy to be disrupted by early-life seizures, and is thought to be related to granule cell dispersion in MTS-associated TLE. In CHAPTER 2, we described the long-term effects of FS on proliferation and on survival of newborn cells, as well as a possible gender difference.

After cells have divided, they migrate and differentiate, and adopt a specific phenotype. Phenotype is what determines the function of a cell and the circuit this cell is incorporated in. In CHAPTER 3, we investigated whether new cells born immediately after FS developed into neurons, astrocytes, or microglia and if they expressed the neuronal glutamate transporter EAAC1.

Prospective studies in humans, investigating the link between FS and TLE, are difficult because of the long silent period after FS before the first clinical symptoms of TLE emerge. In CHAPTER 4, multimodal MRI was used, comprising of cerebral T2 relaxometry and diffusion tensor imaging (DTI), and hippocampal volume measurements and proton spectroscopy, in combination with histology, to find out if FS cause subtle changes in brain structure and metabolism that may have been missed until now in human MRI studies. 
In CHAPTER 5, we looked in more detail into long-lasting FS-induced changes of the hippocampal expression of the different glutamate transporters GLAST and GLT-1, both present on glia, and EAAC1, present on neurons. We further measured, with EEG, the effects of pharmacologically blocking glutamate transport and the possible development of clinical and electrographical seizures.

In CHAPTER 6, we investigated cognitive and behavioral outcome after FS using different learning tasks and measuring locomotor activity.

Finally, in CHAPTER 7, the results of all experiments are discussed, and their significance placed in a broader perspective. 


\section{References}

1. Fisher, R.S., et al., Epileptic seizures and epilepsy: definitions proposed by the International League Against Epilepsy (ILAE) and the International Bureau for Epilepsy (IBE). Epilepsia, 2005, 46(4), 470-472.

2. ILAE., Proposal for revised clinical and electroencephalographic classification of epileptic seizures. From the Commission on Classification and Terminology of the International League Against Epilepsy. Epilepsia, 1981, 22(4), 489-501.

3. Gutierrez-Delicado, E., Serratosa, J.M., Genetics of the epilepsies. Curr. Opin. Neurol., 2004, 17(2), 147-153.

4. Kwan, P., Brodie, M.J., Early identification of refractory epilepsy. N. Engl. J. Med., 2000, 342(5), 314-319.

5. Kwan, P., Brodie, M.J., Refractory epilepsy: mechanisms and solutions. Expert Rev. Neurother., 2006, 6(3), 397-406.

6. Engel, J., Jr., Introduction to temporal lobe epilepsy. Epilepsy Res., 1996, 26(1), 141-150.

7. Ojemann, G.A., Temporal lobe epilepsy -current wisdom. Stereotact. Funct. Neurosurg., 2001, 77(1-4), 213-215.

8. Mathern, G.W., et al., The pathogenic and progressive features of chronic human hippocampal epilepsy. Epilepsy Res., 1996, 26(1), 151-161.

9. Wyler, A.R., et al., A grading system for mesial temporal pathology (hippocampal sclerosis) from anterior temporal lobectomy. J. Epilepsy, 1992, 5, 220-225.

10. Blumcke, I., Thom, M., Wiestler, O.D., Ammon's horn sclerosis: a maldevelopmental disorder associated with temporal lobe epilepsy. Brain Pathol., 2002, 12(2), 199-211.

11. Fisher, P.D., Sperber, E.F., Moshe, S.L., Hippocampal sclerosis revisited. Brain Dev., 1998, 20(8), 563-573.

12. Liu, Z., Mikati, M., Holmes, G.L., Mesial temporal sclerosis: pathogenesis and significance. Pediatr. Neurol., 1995, 12(1), 5-16.

13. Thom, M., et al., Cell proliferation and granule cell dispersion in human hippocampal sclerosis. J. Neuropathol. Exp. Neurol., 2005, 64(3), 194-201.

14. Lewis, D.V., Losing neurons: selective vulnerability and mesial temporal sclerosis. Epilepsia, 2005, 46(Suppl. 7), 39-44.

15. Buckmaster, P.S., Zhang, G.F., Yamawaki, R., Axon sprouting in a model of temporal lobe epilepsy creates a predominantly excitatory feedback circuit. J. Neurosci., 2002, 22(15), 66506658.

16. Haas, C.A., et al., Role for reelin in the development of granule cell dispersion in temporal lobe epilepsy. J. Neurosci., 2002, 22(14), 5797-5802.

17. Bote, R.P., et al., Hippocampal sclerosis: histopathology substrate and magnetic resonance imaging. Semin. Ultrasound CT MR, 2008, 29(1), 2-14. 
18. Ben-Ari, Y., Basic developmental rules and their implications for epilepsy in the immature brain. Epileptic Disord., 2006, 8(2), 91-102.

19. Brooks-Kayal, A.R., Rearranging receptors. Epilepsia, 2005, 46(Suppl. 7), 29-38.

20. Furuta, A., Rothstein, J.D., Martin, L.J., Glutamate transporter protein subtypes are expressed differentially during rat CNS development. J. Neurosci., 1997, 17(21), 8363-8375.

21. Ben-Ari, Y., Holmes, G.L., Effects of seizures on developmental processes in the immature brain. Lancet Neurol., 2006, 5(12), 1055-1063.

22. Offringa, M., Hazebroek-Kampschreur, A.A., Derksen-Lubsen, G., Prevalence of febrile seizures in Dutch schoolchildren. Paediatr. Perinat. Epidemiol., 1991, 5(2), 181-188.

23. Shinnar, S., Glauser, T.A., Febrile seizures. J. Child Neurol., 2002, 17(Suppl. 1), S44-52.

24. ILAE., Guidelines for epidemiologic studies on epilepsy. Commission on Epidemiology and Prognosis, International League Against Epilepsy. Epilepsia, 1993, 34(4), 592-596.

25. Forsgren, L., et al., A prospective incidence study of febrile convulsions. Acta Paediatr. Scand., 1990, 79(5), 550-557.

26. Nelson, K.B., Ellenberg, J.H., Prognosis in children with febrile seizures. Pediatrics, 1978, 61(5), 720-727.

27. Ellenberg, J.H., Nelson, K.B., Febrile seizures and later intellectual performance. Arch. Neurol., 1978, 35(1), 17-21.

28. Verity, C.M., Greenwood, R., Golding, J., Long-term intellectual and behavioral outcomes of children with febrile convulsions. N. Engl. J. Med., 1998, 338(24), 1723-1728.

29. Hirtz, D.G., et al., Does phenobarbital used for febrile seizures cause sleep disturbances? Pediatr. Neurol., 1993, 9(2), 94-100.

30. Kolfen, W., Pehle, K., Konig, S., Is the long-term outcome of children following febrile convulsions favorable? Dev. Med. Child Neurol., 1998, 40(10), 667-671.

31. Berg, A.T., Shinnar, S., Unprovoked seizures in children with febrile seizures: short-term outcome. Neurology, 1996, 47(2), 562-568.

32. Nelson, K.B., Ellenberg, J.H., Predictors of epilepsy in children who have experienced febrile seizures. N. Engl. J. Med., 1976, 295(19), 1029-1033.

33. Berg, A.T., et al., Predictors of recurrent febrile seizures. A prospective cohort study. Arch. Pediatr. Adolesc. Med., 1997, 151(4), 371-378.

34. Camfield, P., et al., What types of epilepsy are preceded by febrile seizures? A populationbased study of children. Dev. Med. Child Neurol., 1994, 36(10), 887-892.

35. Cendes, F., Febrile seizures and mesial temporal sclerosis. Curr. Opin. Neurol., 2004, 17(2), 161-164.

36. Cendes, F., et al., Early childhood prolonged febrile convulsions, atrophy and sclerosis of mesial structures, and temporal lobe epilepsy: an MRI volumetric study. Neurology, 1993, 43(6), 1083-1087.

37. Falconer, M.A., Serafetinides, E.A., Corsellis, J.A., Etiology and Pathogenesis of Temporal Lobe Epilepsy. Arch. Neurol., 1964, 10, 233-248. 
38. Kuks, J.B., et al., Hippocampal sclerosis in epilepsy and childhood febrile seizures. Lancet, 1993, 342(8884), 1391-1394.

39. Knudsen, F.U., Febrile seizures--treatment and outcome. Brain Dev., 1996, 18(6), 438-449.

40. Carratala, F., Moya, M., Febrile convulsions induced by microwaves and the alteration in behavior of albino mouse OF1. Biol. Neonate, 1991, 60(1), 62-68.

41. Hjeresen, D.L., Diaz, J., Ontogeny of susceptibility to experimental febrile seizures in rats. Dev. Psychobiol., 1988, 21(3), 261-275.

42. Werboff, J., Havlena, J., Febrile Convulsions in Infant Rats, and Later Behavior. Science, 1963, 142, 684-685.

43. Nagaki, S., et al., The role of vasopressin, somatostatin and GABA in febrile convulsion in rat pups. Life Sci., 1996, 58(24), 2233-2242.

44. Holtzman, D., Obana, K., Olson, J., Hyperthermia-induced seizures in the rat pup: a model for febrile convulsions in children. Science, 1981, 213(4511), 1034-1036.

45. Jiang, W., Duong, T.M., de Lanerolle, N.C., The neuropathology of hyperthermic seizures in the rat. Epilepsia, 1999, 40(1), 5-19.

46. Ilbay, G., Sahin, D., Ates, N., Changes in blood-brain barrier permeability during hot waterinduced seizures in rats. Neurol. Sci., 2003, 24(4), 232-235.

47. Baram, T.Z., Gerth, A., Schultz, L., Febrile seizures: an appropriate-aged model suitable for long-term studies. Brain Res. Dev. Brain Res., 1997, 98(2), 265-270.

48. Morimoto, T., et al., Hyperthermia-induced seizures with a servo system: neurophysiological roles of age, temperature elevation rate and regional GABA content in the rat. Brain Dev., 1990, 12(3), 279-283.

49. Baram, T.Z., Animal models for febrile seizures, in Febrile seizures, Baram, T.Z. and Shinnar, S., Editors. 2002, Academic Press: London.

50. Dube, C.M., et al., Fever, febrile seizures and epilepsy. Trends Neurosci., 2007, 30(10), 490496.

51. Scott, R.C., et al., Hippocampal abnormalities after prolonged febrile convulsion: a longitudinal MRI study. Brain, 2003, 126(11), 2551-2557.

52. Scott, R.C., et al., Prolonged febrile seizures are associated with hippocampal vasogenic edema and developmental changes. Epilepsia, 2006, 47(9), 1493-1498.

53. VanLandingham, K.E., et al., Magnetic resonance imaging evidence of hippocampal injury after prolonged focal febrile convulsions. Ann. Neurol., 1998, 43(4), 413-426.

54. Fernandez, G., et al., Hippocampal malformation as a cause of familial febrile convulsions and subsequent hippocampal sclerosis. Neurology, 1998, 50(4), 909-917.

55. Germano, I.M., et al., Neuronal migration disorders increase susceptibility to hyperthermiainduced seizures in developing rats. Epilepsia, 1996, 37(9), 902-910.

56. Scantlebury, M.H., et al., Febrile seizures in the predisposed brain: A new model of temporal lobe epilepsy. Ann. Neurol., 2005, 58(1), 41-49. 


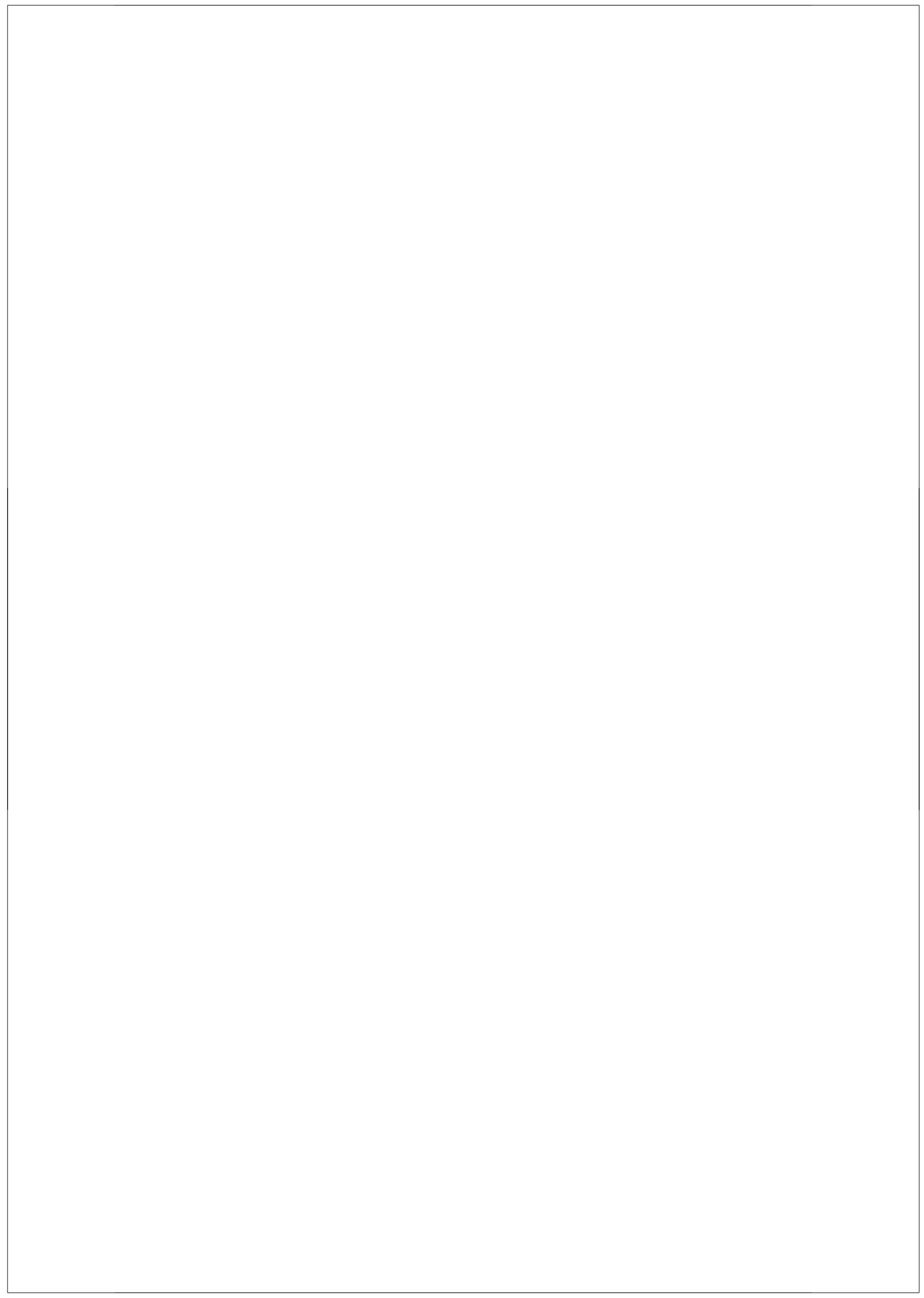




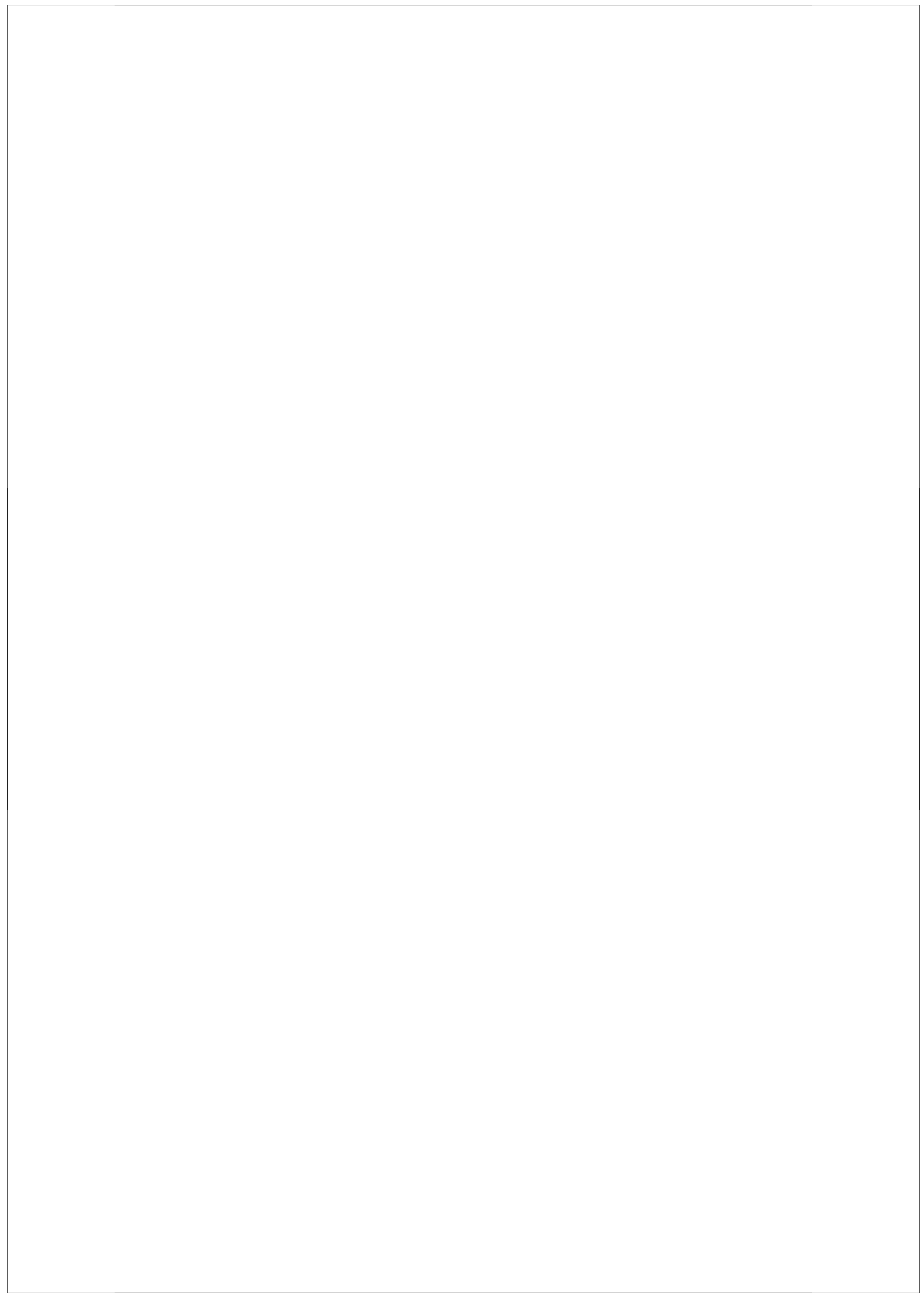



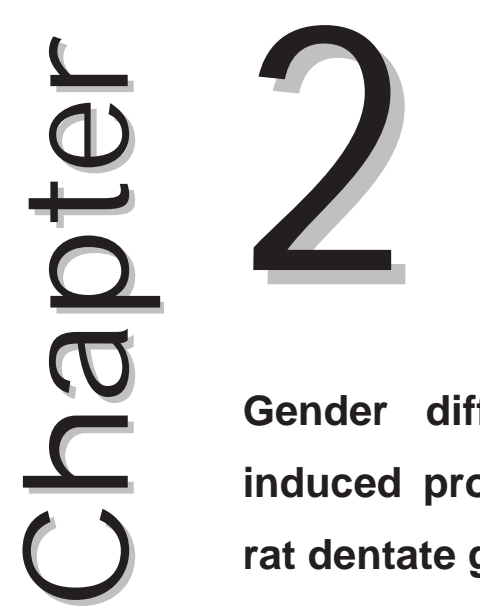

Gender differences in febrile seizureinduced proliferation and survival in the rat dentate gyrus

Evi M.P. Lemmens, Tim Lubbers, Olaf E.M.G. Schijns, Emile A.M. Beuls, Govert Hoogland

Epilepsia, 2005, 46 (10), 1603-1612.

ABSTRACT

Febrile seizures are fever-associated early-life seizures that are thought play a role in the development of epilepsy. Seizure-induced proliferation of dentate granule cells has been demonstrated in several adult animal models, and is thought to be an integral part of epileptogenesis. The aim of the present study was to investigate proliferation and survival of dentate gyrus (DG) cells born after early-life hyperthermia (HT)-induced seizures in male and female rats.

At postnatal day (PN) 10, male and female rats were exposed to heated air to induce seizures. Littermates were used as normothermia controls. Convulsive behavior was observed by two researchers. From PN11 to PN16, rats were injected with bromodeoxyuridine (BrdU) to label dividing cells. The number of BrdU-immunoreactive cells in the DG was counted at PN17 and PN66.

At PN17, male as well as female HT rats had the same amount of BrdU-positive cells compared with controls. At PN66, there were significantly more BrdU-positive cells left in HT females (53\%) than in controls (44\%, percentage of BrdU-positive cells at PN17), whereas there was no difference between HT males and male controls. The net result of proliferation and survival at PN66 was that female HT rats had the same number of BrdU-immunoreactive cells as controls, while male HT rats had $25 \%$ more BrdU-immunoreactive cells than controls $(p<0.05)$.

Early-life seizures cause a sexually dimorphic cytogenic response, that results in an increased population of newborn DG cells in young adult males, while leaving that of young adult females unaltered. 


\section{Introduction}

Febrile seizures (FS) are the most common seizure type in young children. They occur in 3-4\% of children between the age of three months and five years, and are associated with a febrile illness. A number of genetic defects have been associated with a higher incidence of FS, the so-called channelopathies, mutations in genes coding for ion channels, representing the largest group [1-3]. Also environmental factors have been found to increase the risk of developing FS. For instance, in a population-based study Lieberman et al. [4] found almost a fourfold increase in the risk of $\mathrm{FS}$, when maternal fever of more than $100.4^{\circ} \mathrm{F}$ (or $38^{\circ} \mathrm{C}$ ) was measured during labor.

Although a number of factors contributing to FS have been identified, there is still much debate on the consequences. Some studies suggest that FS are benign, causing no damage to the child [5-7]. However, this idea is based on studies that are limited by the use of non-invasive techniques, the follow-up time, and often did not classify the types of epilepsy. In a prospective study, Tarkka et al. [8] failed to show structural or behavioral differences in children that experienced FS when comparing them with age-matched, seizure-free controls. In contrast, Cendes et al. [9] found a 10-fold increased incidence of FS (40\%) among patients with mesial temporal sclerosis associated temporal lobe epilepsy (TLE). Similarly, Camfield et al. [10] found an 8.8 fold increased risk for developing intractable epilepsy after prolonged FS. The suggestion that FS are causally related to the development of TLE is in line with the long-standing notion that "seizures beget seizures". A number of animal models for FS have been developed to study whether FS induce epileptogenesis. Most animal models for FS aim to mimic fever by increasing the body temperature, which can be achieved by exposing the animals to a heat source such as a microwave [11,12], infrared irradiation [13], or warm water [14]. In the present study, we used the immature rat model developed by Baram et al. [15]. The main advantage of this model is that it uses rats during a brain development age 
comparable to that of a human child when it is most susceptible to seizures. Furthermore, the mortality and morbidity is low, which makes this model highly suitable for long-term follow-up studies.

None of the animal studies that used the model of Baram could conclude that hyperthermia $(\mathrm{HT})$-induced seizures cause TLE. They were unable to find histological evidence for the presence of TLE. For instance, hippocampal cell death, a histopathological hallmark of TLE, was not seen in these animals. Still, acute cell damage, evident by argyrophilic neurons, was present but there was no change in the total number of neurons [16]. Chen et al. [17] showed that HTinduced seizures render the hippocampal network hyperexcitable by raising the depolarization current of neurons, thereby lowering the seizure threshold. Previously, daytime behavioral and electrophysiological monitoring failed to demonstrate spontaneous seizures in adult rats after early-life HT-induced seizures. However, these animals had a lower threshold for seizures induced by administration of a subconvulsive dose of kainic acid [18]. Recently, Baram et al. [19] have shown that HT-induced seizures are able to elicit spontaneous nocturnal seizures. Another important finding is increased mossy fiber sprouting after HT-induced seizures which might also contribute to increased excitability of hippocampal neurons [20].

The past few years, altered neurogenesis has been proposed as a mechanism by which seizures modulate the hippocampal network. Several studies show that pilocarpine- or [21-23] kainate-induced seizures [24,25], perforant pathway stimulation [25], or amygdala kindling $[26,27]$ cause an increased proliferation of neurons in adult rats. Most of the newborn cells differentiate into neurons in the dentate granule cell layer and form connections with the CA3 area of the hippocampus and other dentate granule cells [21].

Gender differences in cell proliferation, differentiation, and survival $[28,29]$ have been attributed to the role of hormones in neurogenesis [30]. There is also evidence of gender differences in seizure susceptibility. Males are found to be more susceptible to temporal lobe-like seizures due to high levels of 
testosterone [31]. Also, after administration of the convulsant N-methyl-Daspartate, male rats show more severe seizure activity than female rats [32]. Hence, the aim of the present study was to investigate proliferation of DG cells after early-life $\mathrm{HT}$-induced seizures in male and female rats. 


\section{Materials and M ethods}

\section{Animals}

Sprague-Dawley rats (Harlan, The Netherlands) were housed under standard conditions $\left(21 \pm 2^{\circ} \mathrm{C}\right.$ ambient temperature, a 12-hour light/dark schedule, background noise provided by a radio, food and water ad libitum). A total of 21 male and 24 female rats were divided each into the following four groups: 1. short-term normothermia (ST-NT), sacrificed at postnatal day (PN) 17 ( $n=5$ males, $n=5$ females); 2 . short-term hyperthermia (ST-HT; $n=6$ males, $n=6$ females); 3. long-term normothermia (LT-NT), sacrificed at PN66 ( $n=4$ males, $n=6$ females); and 4. long-term hyperthermia ( $L T-H T ; n=6$ males, $n=7$ females). Rats were weighed daily from PN1-17, and once at PN66. All experiments were approved by the Animal Experiments Committee (DEC) of the University of Maastricht, The Netherlands.

\section{Hyperthermia paradigm}

HT was induced as described previously by Baram [15], with minor modifications. Briefly, PN10 rat pups were injected subcutaneously with $0.2 \mathrm{ml}$ $0.9 \%$ saline to prevent dehydration, and placed in a perspex cylinder with a diameter of $10 \mathrm{~cm}$ (one rat/cylinder). An adjustable stream of heated air $\left(50-52^{\circ} \mathrm{C}\right)$ was blown into the cylinder by a commercial hair dryer, placed at $50 \mathrm{~cm}$ above the rats, to raise the body temperature of the rat pups from a basal value of about $35^{\circ} \mathrm{C}$ to $41-42.5^{\circ} \mathrm{C}$. Core temperatures were measured before and every $2.5 \mathrm{~min}$ during the $\mathrm{HT}$ treatment, with a rectal probe (K-type thermocouple bead probe connected to a ST-9612 digital thermometer, Velleman Components, Gavere, Belgium). When the core temperature of the rats reached $39.5^{\circ} \mathrm{C}$, usually after $5-10 \mathrm{~min}$, the temperature and volume of the air were adjusted so that a core temperature of $41-42.5^{\circ} \mathrm{C}$ was maintained for $30 \mathrm{~min}$. If the core temperature exceeded $42.5^{\circ} \mathrm{C}$, the rat was removed from the cylinder until the temperature dropped to $40-42.5^{\circ} \mathrm{C}$. The occurrence of seizures 
was monitored behaviorally by two observers. The behavioral seizures were stereotyped and previously shown to correlate with EEG discharges in the hippocampus $[15,18]$. Behaviorally, the seizures consisted of arrest of heatinduced hyperkinesis, followed by body flexion, and occasionally followed by clonic contractions of the limbs. The moment the rats showed body flexion was taken as the start of a seizure, while the end of the seizure was marked either by the end of the 30 min hyperthermia treatment or by regaining normal behavior. These time values were used to estimate the average seizure duration. Immediately after the 30 min treatment, the rats were placed in water (room temperature, RT) with their head above the surface to regain their normal body temperature, after which they were returned to their mother.

Normothermia (NT) controls from the same litter as the HT rats were exposed to the same conditions, except that the stream of air was used to maintain the core temperature of the rats. At PN22, all pups were weaned and randomly housed two to three per cage. A schematic overview of the experimental design is presented in figure 1.

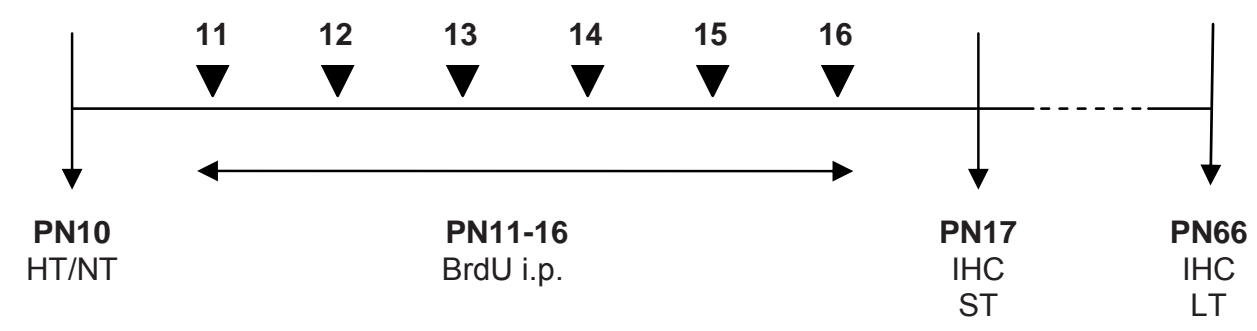

FIG. 1. Time schedule of the experimental design. At postnatal day (PN) 10, rats received a hyperthermia (HT) or normothermia (NT) treatment. From PN11-16, rats were injected twice daily, intraperitoneally (i.p.), with $25 \mathrm{mg}$ bromodeoxyuridine (BrdU)/kg body weight. Immunohistochemical (IHC) staining for BrdU was carried out at PN17 (short-term, ST) or PN66 (long-term, LT). 


\section{BrdU immunohistochemistry}

From PN11-16, all rats received twice daily (minimum six hours apart) intraperitoneal injections of the thymidine analog 5'bromo-2'deoxyuridine (BrdU, $25 \mathrm{mg} / \mathrm{kg}$, Sigma, St. Louis, MO, USA), $2 \mathrm{mg} / \mathrm{ml}$ in $0.9 \%$ saline ( $\mathrm{pH} \mathrm{7.6).} \mathrm{At}$ PN17 or PN66, the rats received an overdose of Nembutal ${ }^{\circledR}$, followed by perfusion fixation with $4 \%$ paraformaldehyde in $0.1 \mathrm{M}$ phosphate buffer (PB, $\mathrm{pH}$ 7.6). After the brains were removed, they were postfixed in $4 \%$ paraformaldehyde/0.1 M PB for $48 \mathrm{~h}\left(4^{\circ} \mathrm{C}\right)$, and cryoprotected in $20 \%$ sucrose/0.1 M PB for $\left(24 \mathrm{~h}, 4^{\circ} \mathrm{C}\right)$. Coronal serial sections of $10 \mu \mathrm{m}$ were cut on a cryostat, and six sections per rat were mounted on Superfrost ${ }^{\circledR}$ slides. The first and last sections were maximally $100 \mu \mathrm{m}$ apart from each other.

For BrdU detection, the sections were washed in TBS $(0.1 \mathrm{M}$ Tris-base, $0.15 \mathrm{M} \mathrm{NaCl}, \mathrm{pH} 7.4$ ), treated with TBS containing $0.6 \% \mathrm{H}_{2} \mathrm{O}_{2}$ for $30 \mathrm{~min}$, and washed again in TBS (2x $10 \mathrm{~min}$ at $\mathrm{RT}, 1 \times 10 \mathrm{~min}$ at $65^{\circ} \mathrm{C}$ ). For DNA denaturation, the sections were incubated in $50 \%$ formamide/2x SSC buffer $\left(0.3 \mathrm{M} \mathrm{NaCl}, 0.03 \mathrm{M}\right.$ sodium citrate, $\mathrm{pH} 7.0$ ) for 2 hours at $65^{\circ} \mathrm{C}$, rinsed in 2x SSC buffer (RT), incubated in $2 \mathrm{~N} \mathrm{HCl}$ for 30 min at $37^{\circ} \mathrm{C}$, and incubated in $0.1 \mathrm{M}$ borate buffer $(\mathrm{pH} \mathrm{8.5)}$ for $10 \mathrm{~min}$ (RT). After this pretreatment, the sections were washed six times in TBS, incubated in TBS-TS (TBS containing $0.25 \%$ Triton $\mathrm{X}-100$ and 3\% normal donkey serum) for $60 \mathrm{~min}(\mathrm{RT})$, and then incubated overnight in primary anti-BrdU antibody (monoclonal mouse; Roche, Almere, The Netherlands; 1:800 in TBS-TS).

After rinsing the sections in TBS, they were incubated for $1 \mathrm{~h}$ in secondary biotinylated donkey anti-mouse antibody (Jackson Immunoresearch Laboratories, West Grove, PA, USA; 1:400 in TBS-TS). The staining was visualized with a Vectastain $\mathrm{ABC} /$ Elite standard kit (Vector Laboratories, Burlingame, CA, USA), based on the avidin-biotin-peroxidase reaction, with diaminobenzidine as chromogen, and $\mathrm{NiCl}_{2}$ as a signal enhancer. Finally, the sections were counterstained with $0.2 \%$ cresyl violet for $1 \mathrm{~h}$, dehydrated, and 
coverslipped with DePeX. Sections of the NT and HT groups from both genders were processed simultaneously to minimize inter-assay variability.

\section{Quantitative analysis}

Per rat, six coronal sections cut between Bregma -2.12 and $-2.30 \mathrm{~mm}$ were used as separate values for quantitative analysis of the BrdU staining. This analysis was carried out using an Olympus BX51 microscope coupled to a computer supported by the Stereo Investigator program (MicroBrightField Inc., Williston, VT, U.S.A.). In each section, the left or right DG (randomly chosen) was delineated as depicted in figure $2 \mathrm{~A}$, the number of BrdU-positive cells in the delineated area was counted, and the surface of that area was calculated by the Stereo Investigator program. A cell was included if it was uniformly or fragmentally stained (see fig. 2B) and had the morphologic appearance of an oval or round nucleus. The reason for counting fragmentally stained cells is that the BrdU label dilutes with each cell division [33]. The number of BrdU-positive cells per $100,000 \mu \mathrm{m}^{2}$ was calculated, and the cell counts of the HT groups were also expressed as percentage of NT (mean of $100 \%$ ). Cell counts were performed at a 500x magnification by two observers blinded to the treatmentstatus of the sections, and showed no significant inter-observer variation.

To avoid bias of potential changes due to the HT treatment or gender, the size of the DG (mean surface area of six sections) as well as the size of the cells (mean of 100 BrdU-labeled cells per rat) and cell density of the dentate granule cell layer (DGL, total of BrdU-labeled and cresyl violet-stained cells) were measured using the Stereo Investigator program (MicroBrightField Inc., Williston, VT, U.S.A.). 

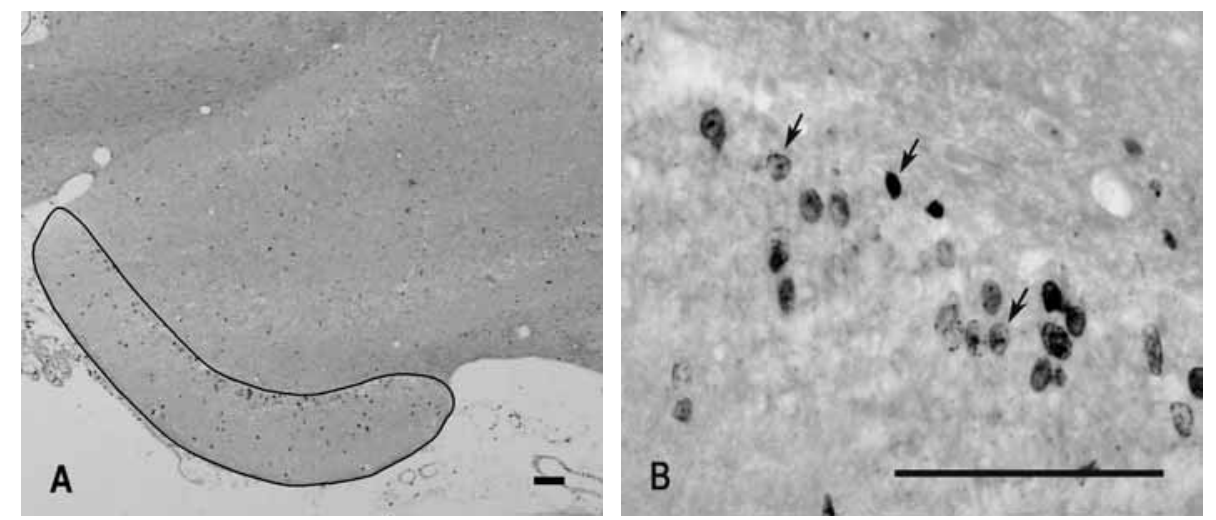

FIG. 2. Representative photomicrographs of a coronal section of the hippocampus of a 66-day old male control rat. After immunohistochemical detection of $\mathrm{BrdU}$, the section was counterstained with cresyl violet. (A) The dotted line represents the delineation of the dentate gyrus (DG). (B) Detailed picture of the granule cell layer of the DG. Cells that were uniformly or fragmentally stained (soccer ball pattern) were counted (arrows). Scale bars $=100 \mu \mathrm{m}$.

\section{Statistical analysis}

Body weight measurements and growth curves were analyzed using repeated measures analysis of variance (ANOVA) and Bonferroni's multiple comparison post hoc test. To analyze differences in body weight between treatment groups but within gender groups at each individual day, a Student $t$ test was used. Treatment, gender, and time effect on the number of BrdU-labeled cells, cell size, surface area of the DG and cellular density were analyzed using ANOVA with Bonferroni's post hoc test. Data are presented as mean \pm SEM. Significance levels were set at $p<0.05$. 


\section{Results}

In the present study, the effect of HT-induced seizures on proliferation and survival of DG cells was determined in immature male and female rats.

At the end of the HT phase, all HT rats displayed severe behavioral seizures, characterized by arrest of the heat-induced hyperkinesis, followed by body flexion and occasionally by clonic movements of the limbs. The average seizure duration in males $(7.86 \pm 0.81 \mathrm{~min})$ was not significantly different from that in females $(10 \pm 1.54 \mathrm{~min})$. None of the NT rats had behavioral seizures or showed abnormal behavior during their stay in the cylinder.

Body weight measurements showed that the HT treatment had no overall effect on the growth curve (from PN12 onwards, the slope of NT rats is not significantly different from that of $\mathrm{HT}$ rats) but did reduce weight gain in the first 1-2 days post-HT (fig. 3). ANOVA showed an interaction between day and treatment when body weights from PN10-16 were analyzed $\left(F_{6,246}=5.42\right.$, $\mathrm{p}<0.001)$, but from $\mathrm{PN} 12-16$ this interaction was not present. Although pretreatment body weights of $\mathrm{HT}$ and NT rats were the same, the post-HT dip in weight gain resulted in a transiently reduced body weight of HT rats compared with their NT counterpart $\left(F_{1,41}=13.32, p<0.01\right)$. From PN10-16, there was no overall effect of gender and there was also no interaction between gender and treatment on body weight.

At PN66, the treatment effect was no longer present but then a gender effect appeared $\left(F_{1,14}=499.24, p<0.001\right)$ in which male rats weighed significantly more than female rats within each treatment group (post hoc; NT: male $279 \pm 4 \mathrm{~g}$ vs. female $177 \pm 4.43 \mathrm{~g}, \mathrm{p}<0.001 ; \mathrm{HT}$ : male $282 \pm 2.71 \mathrm{~g}$ vs. female $176 \pm 4.42 \mathrm{~g}, \mathrm{p}<0.001)$. 

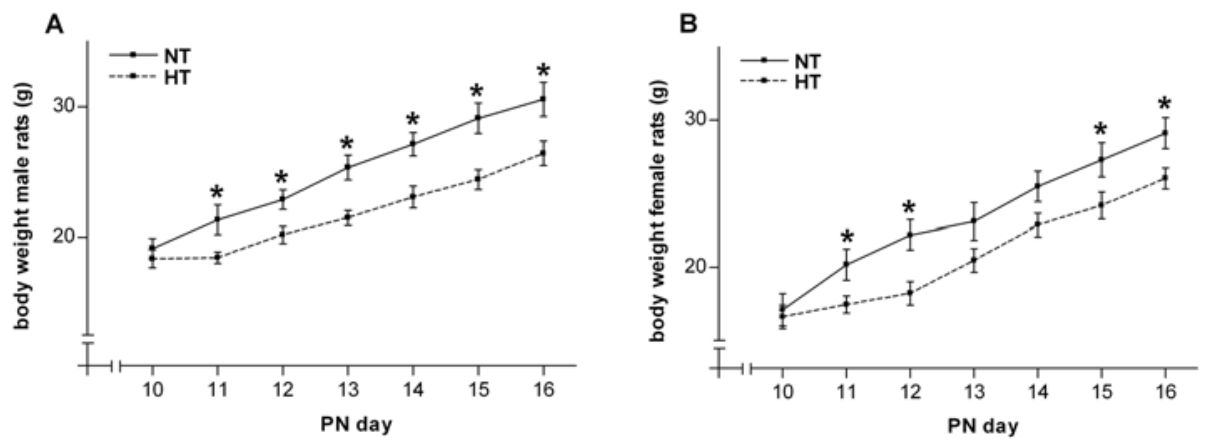

FIG. 3. Growth curves from postnatal (PN) day 10-16. Mean body weight of (A) male normothermia $(N T, n=9)$ and hyperthermia rats $(H T, n=12)$, and of $(B)$ female NT $(n=11)$ and $H T(n=13)$ rats. Though pretreatment (PN10) body weights of NT and HT rats did not differ, post-treatment (PN1116) body weights of HT rats were significantly reduced compared with their NT counterparts. From PN12-16, weight gain of NT rats was the same as that of HT rats (slopes of the NT and HT curves are not significantly different). Data are expressed as mean $\pm \mathrm{SEM},{ }^{*} \mathrm{p}<0.05$ (difference between NT and $\mathrm{HT}$, Student $t$ test).

To determine the effect of HT-induced seizures on proliferation and on survival of newborn cells, we labeled post-seizure dividing cells with BrdU, and quantified the number of BrdU-immunoreactive cells either shortly after labeling (i.e. at PN17) or seven weeks after the last BrdU injection (i.e. at PN66). Figure 4 shows typical examples of the BrdU staining patterns found in the DG of the different treatment groups. They illustrate a clear decrease in BrdU labeling with increasing age (ST versus LT). Only pictures of the male groups are presented since male and female BrdU staining patterns looked alike. 

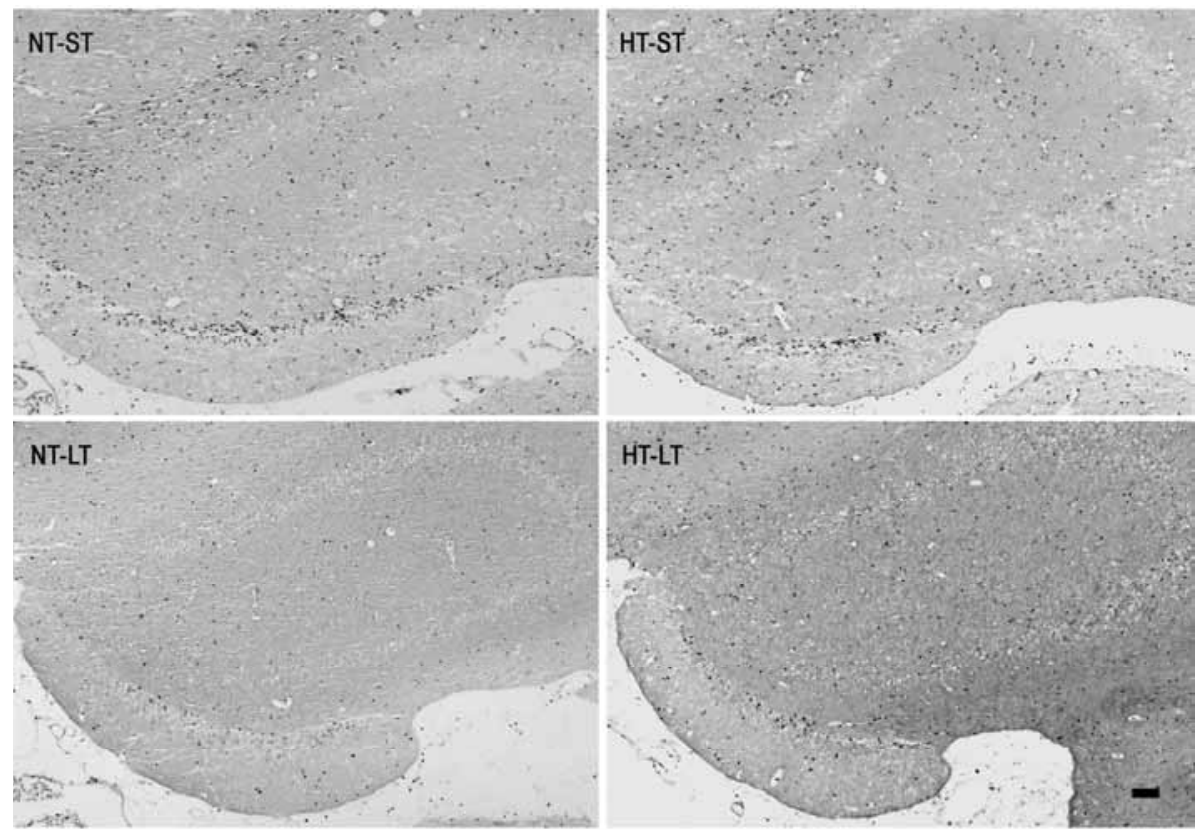

FIG. 4. Photomicrographs of BrdU immunolabeling in the dentate gyrus (DG) of the male rats. The sections were counterstained with cresyl violet to visualize the hippocampal structure. Fifty days after the last BrdU injection (LT) there is an unambiguous decrease in BrdU labeling in the DG compared with labeling one day after the last injection (ST). BrdU staining patterns were similar in the female groups (pictures not shown). NT, normothermia; HT, hyperthermia; ST, short-term, PN17; LT, long-term, PN66. Scale bar $=250 \mu \mathrm{m}$.

BrdU quantification revealed that HT induces a gender-specific cytogenic response in the DG. To be able to compare BrdU counts between the present study and others, raw data of the number of BrdU-labeled cells $/ 100,000 \mu \mathrm{m}^{2}$ are as follows: at PN17, male NT $=116.13 \pm 3.22$, male $\mathrm{HT}=126.73 \pm 4.6$, female $\mathrm{NT}=79.21 \pm 2.35$, female $\mathrm{HT}=71.46 \pm 2.35$; at PN66, male NT $=23.09 \pm 1.79$, male $\mathrm{HT}=29.21 \pm 1.66$, female NT $=34.73 \pm 2.15$, female $\mathrm{HT}=37.78 \pm 1.67$. At PN17 female NT and HT rats had significantly less BrdU-labeled cells per $100,000 \mu \mathrm{m}^{2}$ than their male counterparts (ANOVA, $p<0.001$ for each treatment group). At PN66, there was no gender effect. Notice the decrease in BrdU labeling at PN66 compared with PN17. ANOVA showed a significant time effect 
within each gender (ANOVA, $p<0.001$ for each gender). The data of the NT groups are also presented in figure 5 .

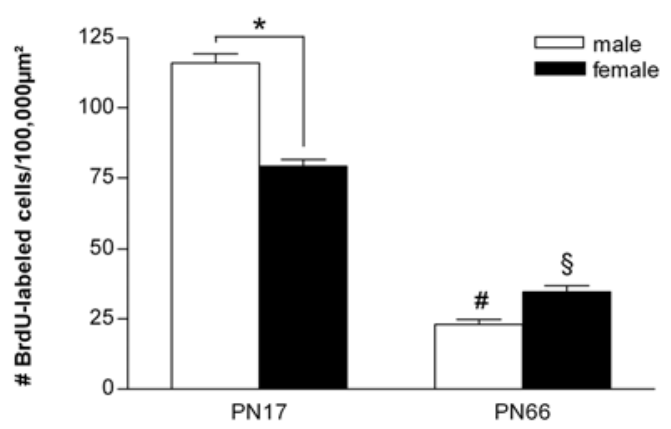

FIG. 5. Absolute number of BrdU-positive cells in the normothermia controls. At PN17, female rats had significantly less BrdU-positive cells than male rats, while at PN66 this gender difference was no longer present. When comparing the number of BrdU-labeled cells at PN66 with that at PN17, a significant decrease is observed in the male and female group (PN17, male: $n=5$ and female: $n=5$; PN66, male: $n=4$ and female: $n=7)$. Data are presented as mean \pm SEM $\left({ }^{*}\right.$ : male vs. female at PN17, p<0.001; \#: male PN66 vs. PN17, p<0.001; §: female PN66 vs. PN17, $p<0.001$ ).

In order to compare the HT effect between genders, the number of BrdUlabeled cells $/ 100,000 \mu \mathrm{m}^{2}$ of the HT groups is presented as percentage of their control group in which the control groups have a mean of $100 \%$ (fig. 6). At PN17, male and female HT rats had the same percentage of newborn cells as their controls. 


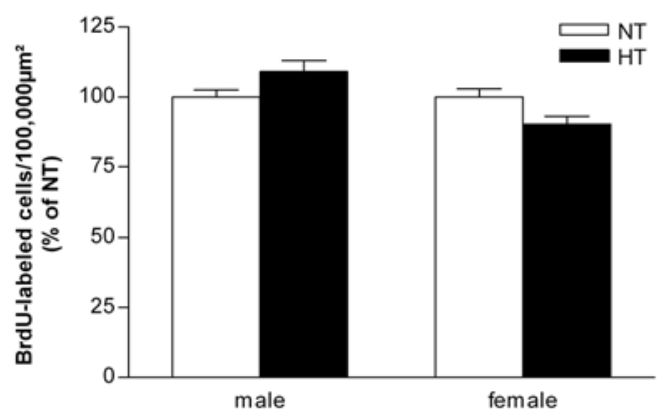

FIG. 6. BrdU quantification in the dentate gyrus at PN17. After hyperthermia (HT) treatment, male and female rats showed the same percentage of BrdU-positive cells as their normothermia (NT) control rats (male: NT, $n=5 / H T, n=6$; female: $N T, n=5 / H T, n=6$ ). Data are presented as mean \pm SEM.

To compare gender differences in survival, we expressed the number of BrdUpositive cells at PN66 as a percentage of the number of BrdU-positive cells counted at PN17 (fig. 7). From all the newborn cells at PN17 that were BrdU labeled during the first six days following treatment, seven weeks later there were $23 \%$ left in HT males and $20 \%$ in NT males. In females however, $53 \%$ survived in HT rats compared with $44 \%$ in NT rats (ANOVA with post hoc, $p<0.05)$.

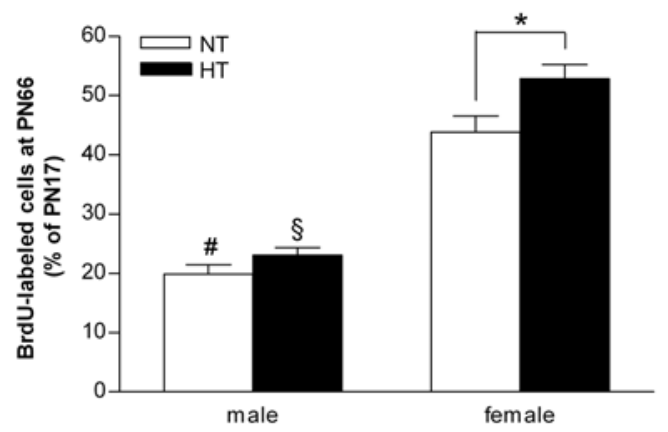

FIG. 7. Survival of newborn cells at PN66. Male hyperthermia (HT) rats showed no difference in surviving cells compared with male normothermia (NT) controls, while female HT rats had significantly more surviving cells than female NT rats (male: NT, $n=4 / H T, n=6$; female: $N T, n=6 / H T$, $n=7$ ). Data are presented as mean \pm SEM. (\#: male NT vs. female NT, $p<0.001$; $\S$ : male HT vs. female HT, $p<0.001$; *: female NT vs. female HT, $p<0.05$ ). 
BrdU quantification at PN66 (fig. 8) shows the net result of proliferation and survival. About $25 \%$ more BrdU-positive cells were found in the DG of male HT rats than in the DG of male NT rats (ANOVA with post hoc, $p<0.05$ ). At this age, the number of BrdU-positive cells did not differ between female HT and female NT rats.

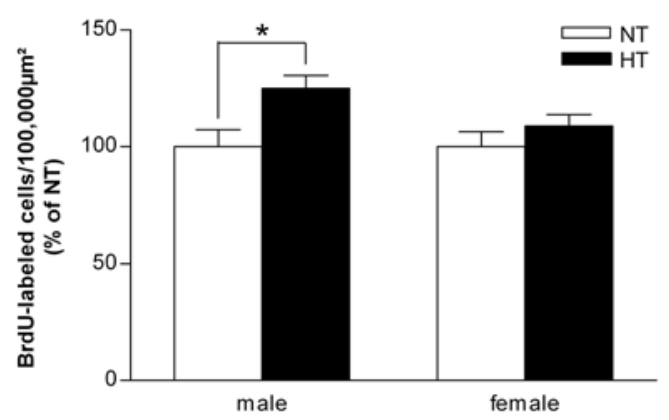

FIG. 8. BrdU quantification in the dentate gyrus at PN66. The net result of proliferation and survival showed that male hyperthermia (HT) rats had $25 \%$ more BrdU-positive cells than male normothermia (NT) rats, whereas the amount of BrdU-positive cells did not differ between female HT and NT rats (male: NT, $n=4 / H T, n=6$; female: NT, $n=6 / H T, n=7$ ). Data are presented as mean \pm SEM, ${ }^{*} p<0.05$

To avoid bias from potential changes in DG size, cell size and cell density of the DGL, associated with HT treatment or gender, these three parameters were compared between the groups. The results were as follows: at PN17, neither HT treatment nor gender had an effect on the surface area of the DG, cell size or cell density; at PN66 HT treatment resulted in a significantly smaller DG surface area in female HT rats compared with female NT rats (ANOVA with post hoc, $p<0.05$ ), whereas in males HT treatment had no effect. Cell size and cell density were not affected by treatment or gender at PN66. With respect to gender differences in growth, PN66 male rats had a larger DG than females irrespective of treatment. When comparing the values from PN66 with PN17, cell density did not differ between any of the groups whereas the surface area of the DG was larger at PN66. This was irrespective of gender and treatment, and was accompanied by an increased cell size at PN66. 


\section{Discussion}

One of the main features of epilepsy is a disturbed balance between excitation and inhibition, resulting in hyperexcitability. There are a number of hippocampal abnormalities, such as mossy fiber sprouting, astrogliosis, and neuronal loss that may account for this hyperexcitability. In addition to these histopathological findings it was recently shown that seizures can alter the number of newborn dentate granule cells [21-27]. This observation has led to the hypothesis that seizure-induced neurogenesis may have a permanent effect on the constituents of the hippocampal network, thereby contributing to the process of epileptogenesis. Here we studied seizure-induced cytogenesis in the DG of developing male and female rats because, under normal physiological conditions, neurogenesis is dependent on factors such as age and sex hormones [31,32].

The major findings from the present study are the following: (a) early-life seizures do not alter cytogenesis in the DG of male or female rats, (b) the survival of DG cells born after early-life seizures is, compared with littermate controls, increased in female rats and unchanged in male rats, and (c) the net result of proliferation and survival is that eight weeks after treatment, HT males had $25 \%$ more BrdU-positive cells than controls, while in females there was no difference between $\mathrm{HT}$ and control rats.

\section{Gender differences in proliferation and survival of cells in normo- thermia controls}

Sex hormones are known to play a role in proliferation and survival. Thus, in order to study whether HT-induced seizures affect these processes, we first investigated gender differences in normothermia controls (fig. 5). The analysis showed that at PN17, female rats had significantly less newborn cells than male rats, whereas this difference was no longer present at PN66. 
The lower proliferation in females found in the present study is in agreement with the results of Perfilieva et al. [29], who injected young naïve SpragueDawley rats with BrdU for seven consecutive days. They also found that one day after the last injection, female rats had significantly less BrdU-labeled DG cells than their male counterparts. Apparently, a gender difference in basal proliferative rate of DG cells exists in immature rats. In line with the present study, they also evaluated the number of BrdU-labeled cells thirty days after the last $\mathrm{BrdU}$ injection and found no difference between males and females. Since PN17 females showed significantly less newborn cells than males, and PN66 females had the same amount of BrdU-positive cells as PN66 males (fig. 5), the survival (PN66 as a percentage of PN17, fig. 7) was significantly higher in females $(44 \%)$ than in males $(20 \%)$. This result is similar to that found by Perfilieva et al. [29].

To our knowledge, gender differences in proliferation and survival have not been studied in rats of comparable age and at time intervals similar to those used in the present study. Although there is some information about the influence of gender on proliferation in adults, the number of studies is limited. A few studies, such as that by Tanapat et al. [28], found a transient increase in the number of newborn cells in adult female rats. This was attributed to increased levels of estrogen since estrogen-treated ovariectomized rats showed an increased proliferation. Because the rats in the present study were treated during the first two weeks of their lives and therefore had not reached sexual maturity when first analyzed at PN17, it is unlikely that a higher estrogen level in the females was the cause of the gender difference in proliferation found here. On the other hand, sex differences in estrogen receptor density, or perhaps binding capacity, might explain our data. However, O'Keefe et al. [34] found no sex differences in the concentration of estrogen receptors or in estradiol receptor binding in young Sprague-Dawley rats. An alternative explanation for the gender difference in proliferation comes from a study by Pang et al. [35]. They found that serum concentrations of testosterone from PN1-10 were about three times higher in male rats than in females, whereas serum levels of 
estrogens did not differ between both genders. Since neonatal administration of testosterone has been found to also increase postnatal cell proliferation [36], this might explain the higher proliferation in males compared with females in our study.

On the contrary, the time point at which survival was analyzed does coincide with the age at which the rats had attained sexual maturity (PN66). Although the molecular mechanisms of survival of newborn cells are not well understood, gender differences suggest that sex hormones are involved in this process as well. For example, estrogen has been found to exert a neuroprotective effect $[37,38]$. Therefore, the increased survival in females may occur as a result of an increase in estrogen levels and consequently a reduced cell death.

\section{Absence of gender differences in seizure-induced proliferation}

Because seizures can alter proliferation and because, as mentioned above, there is a gender difference in basal proliferative rate, we studied the effect of HT-induced seizures on proliferation in both male and female rats. We found no effect of HT-induced seizures on proliferation in either of the two genders (fig. 6). It is known that postnatal neurogenesis can be modified by many factors. Here, we discuss several factors that may have particular importance for the present study.

First, factors related to the treatment, such as seizure duration, weight loss, and stress, may affect the BrdU cell counts. The observation that HT-induced seizures did not alter the birthrate of DG cells one week after the seizures (PN17) is in agreement with the results of a previous study by Bender at al. [20]. The authors suggested that the duration of the HT-induced seizures is insufficient to provoke DG cell proliferation in the immature hippocampus. This idea was based on the observation that long-lasting (> $120 \mathrm{~min}$ ) KA-induced seizures increased the number of newborn cells when compared with littermate controls, and HT-induced seizures were unable to change the number of proliferating cells. However, a difference in newborn cells was present in the 
control groups, i.e. the control group that was used to compare with the KAtreated animals had less BrdU-labeled cells than the control group that was used for comparison with the HT-treated animals. The HT- and KA- treated animals in contrast, showed the same amount of BrdU-labeled cells. Weight loss may also have affected our results. For instance, food deprivation resulting in reduced weight gain is associated with decreased proliferation [39]. In line with the results of Stern et al. [40], we found that HT treatment caused a transiently reduced body weight. This was probably due to a reduced food intake during the first 24 hours after treatment, because from PN12 on HT and control rats showed a similar weight gain, and on PN66 there was no longer a difference in body weight between HT and control animals. More importantly, this effect was similar in male and female rats, and at this early age there was no difference in body weight between male and female controls, which is in agreement with findings of Perfilieva et al. [29]. We thus have no indications that HT-induced weight loss affected our results. Finally, stress is another wellknown factor able to influence the proliferative rate. Stress is known to decrease proliferation in the adult rat $[41,42]$. The stress in the present study might derive from the treatment itself, for instance from maternal separation. Although we cannot exclude that isolation from the mother played a role in the neurogenesis process in these pups, there are several reasons why it is unlikely that the stress induced by isolation affected our findings. Firstly, because the separation during the NT or HT treatment only lasted 30 minutes, while in most studies pups and mothers are separated for 3-24 hours. In these studies it seems that the lack of tactile stimulation by the mother and milk deprivation is altering physiological responses (for review see [43]). During the isolation, we frequently measured body temperature, which involved tactile stimulation, and reduced dehydration by injecting saline prior to separation. Moreover, Severino et al. [44] studied gender differences in stress response at different stages in the animal's lifespan after neonatal handling. They handled the animals from PN1-10 for one minute per day. At PN11 they found no difference in the stress response between males and females. Additionally, and most importantly, male and 
female controls and HT-treated animals were exposed to the same level of stress due to isolation. In addition to maternal separation, seizures may also have caused some stress in this model. Liu et al. showed that suppression of hippocampal neurogenesis in immature rats is associated with the number of seizure episodes and glucocorticosteroid levels [45]. We induced HT-seizures only once and found no change in proliferation. This is in agreement with the results of Liu et al. who also found no change in the number of newborn cells after one seizure episode. To summarize, treatment effects such as seizure duration, weight loss and seizure-induced stress do not seem to have affected proliferation in this model.

A second important factor that may have affected our results is the methodology applied. In the present study, proliferating cells were labeled with BrdU for six consecutive days following the NT or HT treatment. Thus evaluation of the number of BrdU-positive cells one day after the last injection (one week after the treatment) cannot exclude that some newborn cells had already died. It is suggested by Hayes and Nowakowski [33] that a more accurate estimation of pure proliferation can be given when injecting a single dose of BrdU immediately after treatment, followed by evaluation of the number of BrdUpositive cells within 24 hours. However, Bender et al. [20] followed this suggestion and did not find differences in proliferation after HT-induced seizures either. The absence of an altered proliferation rate after HT-induced seizures in the present study might derive from increased cell death accompanying increased proliferation. However, like Bender et al., others have used the HT model to study cell death after seizures and failed to detect increased cell death $[16,20,46]$. We therefore conclude that there was indeed no change in proliferation in our study. To our knowledge, it is not yet known whether gender differences in cell death exist in the HT model for early-life seizures. Furthermore, to avoid bias from potential differences in DG size, cell size and cell density, we analyzed if gender or treatment affected these parameters. We found no overall gender or treatment effect on the DG size, cell size, or cell density at PN17. 


\section{Gender differences in survival of cells born after HT-induced seizures}

This is the first report that has quantified the survival of DG cells born after HTinduced seizures in immature male and female rats. At PN66, male controls had $20 \%$ and $\mathrm{HT}$ males had $23 \%$ BrdU-labeled cells compared with those at PN17. $\mathrm{HT}$-induced seizures also increased the survival of newborn cells in females, since at PN66, female controls had $44 \%$ whereas HT females had $53 \%$ BrdUlabeled cells compared with those at PN17. Thus, HT-induced seizures hardly affected the survival of newborn DG cells in males, and significantly increased that in female rats. At PN66, cell size and cell density were not affected by gender or treatment. Also the DG size was not affected by treatment in males; however female HT rats had a $14 \%$ smaller DG than their NT counterparts. This result is somewhat difficult to explain but is probably not due to the treatment since such a difference was not found at PN17 or in males. Most importantly, cellular density and cell size were the same in both groups, and by visual inspection of cresyl violet-stained sections, we did not find any evidence of damage in the DG of the female HT rats. The most likely explanation therefore is utilization of sections from different levels, with sections from NT females taken somewhat closer to Bregma -2.30 than those of female NT rats. The main limitation of this finding might be that the sample size in the HT group is not as accurate as in the NT group.

Our results showed substantial gender differences in survival. As described earlier, survival of newborn cells in female controls was significantly higher than in male controls. In addition to the gender-related difference in survival, HTinduced seizures caused an even higher increase in the survival rate in females while they did not affect the survival rate in males. This might be explained by the fact that seizures are known to change the level of reproductive hormones (for review: [47,48]) such as a rise in estrogen levels. Recently, Dube et al. [19] showed that adult rats (PN70) develop nocturnal spontaneous seizures after early-life HT-induced seizures. So these spontaneous seizures, if present in our 
rats, may be responsible for the sexually dimorphic survival rate. At PN17, we did not find a different effect of the HT treatment between the two genders. This suggests that the HT-induced seizures at PN10 are not able to affect the levels of sex hormones in a way spontaneous seizures can.

\section{Consequences of altered proliferation and survival after HT- induced seizures}

The net result of proliferation and survival was that eight weeks after HTinduced seizures, female HT rats had similar amounts of BrdU-labeled cells compared with controls, whereas male HT rats had $25 \%$ more BrdU-labeled DG cells than controls. Porter and colleagues [49] have recently assessed the survival of cells born after pilocarpine-induced status epilepticus in PN20 rats, using BrdU injections to label cells dividing 4, 6, and 8 days post-seizure. Three weeks after the last BrdU injection, they also found significantly more BrdUlabeled dentate granule cells in status epilepticus rats than in controls. Further characterization of these surviving cells learned that about $90 \%$ co-expressed the neuronal marker NeuN, and thus were neurons. They also found that the percentage of BrdU-labeled cells that co-expressed NeuN did not differ between control and rats that had experienced seizures. So they concluded that status epilepticus did not alter the neuronal fate of the surviving cells. The observation that seizure-induced cytogenesis in immature rats mostly results in the birth of neurons confirms previous studies [23,50]. A recent study by Dayer et al. [51] suggests that these surviving neurons are very stable, and may permanently replace neurons that are integrated into circuits. Previous studies using the HT model in male rats have failed to detect significant hippocampal neuronal drop out at four weeks after seizures $[16,20,46]$, resulting in the same total number of neurons in controls and seizure rats. Also our own data failed to demonstrate a treatment effect on cell density. Thus, the increased number of BrdU-labeled cells we observed eight weeks after HT-induced seizures in male rats suggests that cell death did occur, but dead cells have been replaced by newborn cells. 
Recently, a review paper by Shapiro and Ribak [52] described a change in the migration pattern of newborn dentate granule cells after seizures. The so-called basal dendrites, which normally retract early in dentate granule cell development, fail to do this after seizures resulting in an ectopic location of these cells in the hilus. This idea was based on the results of several studies where it was shown that seizures caused an increased number of newborn granule cells that resided in the hilus. These ectopic cells, although present in small numbers, are suggested to play a role in hippocampal hyperexcitability seen in epileptic rodents. The functional consequences of the increase in cells in the male HT rats seen in the present study remain to be elucidated, but may be resolved in part by determining the identity and migration of these newborn cells.

To summarize, it was shown that $\mathrm{HT}$-induced seizures do not change proliferation of DG cells in male or female rats. On the other hand, survival of these newborn cells was higher in female than in male rats. The net result is an increased population of newborn DG cells in young adult males while leaving that of young adult females unaltered, which might contribute to gender-related differences in seizure susceptibility. 


\section{References}

1. Baulac, S., Huberfeld, G., Gourfinkel-An, I., et al., First genetic evidence of GABA(A) receptor dysfunction in epilepsy: a mutation in the gamma2-subunit gene. Nat. Genet., 2001, 28(1), 4648.

2. Wallace, R.H., Marini, C., Petrou, S., et al., Mutant ABA(A) receptor gamma2-subunit in childhood absence epilepsy and febrile seizures. Nat. Genet., 2001, 28(1), 49-52.

3. Wallace, R.H., Scheffer, I.E., Parasivam, G., et al., Generalized epilepsy with febrile seizures plus: mutation of the sodium channel subunit SCN1B. Neurology, 2002, 58(9), 1426-1429.

4. Lieberman, E., Eichenwald, E., Mathur, G., Richardson, D., Heffner, L., Cohen, A., Intrapartum fever and unexplained seizures in term infants. Pediatrics, 2000, 106(5), 983-988.

5. Ellenberg, J.H., Nelson, K.B., Febrile seizures and later intellectual performance. Arch. Neurol., 1978, 35(1), 17-21.

6. Nelson, K.B., Ellenberg, J.H., Prognosis in children with febrile seizures. Pediatrics, 1978, 61(5), 720-727.

7. Verity, C.M., Greenwood, R., Golding, J., Long-term intellectual and behavioral outcomes of children with febrile convulsions. N. Engl. J. Med., 1998, 338(24), 1723-1728.

8. Tarkka, R., Paakko, E., Pyhtinen, J., Uhari, M., Rantala, H., Febrile seizures and mesial temporal sclerosis: No association in a long-term follow-up study. Neurology, 2003, 60(2), 215-218.

9. Cendes, F., Andermann, F., Dubeau, F., et al., Early childhood prolonged febrile convulsions, atrophy and sclerosis of mesial structures, and temporal lobe epilepsy: an MRI volumetric study. Neurology, 1993, 43(6), 1083-1087.

10. Camfield, P., Camfield, C., Gordon, K., Dooley, J., What types of epilepsy are preceded by febrile seizures? A population-based study of children. Dev. Med. Child. Neurol., 1994, 36(10), 887-892.

11. Hjeresen, D.L., Diaz, J., Ontogeny of susceptibility to experimental febrile seizures in rats. Dev. Psychobiol., 1988, 21(3), 261-275.

12. Carratala, F., Moya, M., Febrile convulsions induced by microwaves and the alteration in behavior of albino mouse OF1. Biol. Neonate, 1991, 60(1), 62-68.

13. Holtzman, D., Obana, K., Olson, J., Hyperthermia-induced seizures in the rat pup: a model for febrile convulsions in children. Science, 1981, 213(4511), 1034-1036.

14. Jiang, W., Duong, T.M., de Lanerolle, N.C., The neuropathology of hyperthermic seizures in the rat. Epilepsia, 1999, 40(1), 5-19.

15. Baram, T.Z., Gerth, A., Schultz, L., Febrile seizures: an appropriate-aged model suitable for long-term studies. Brain Res. Dev. Brain Res., 1997, 98(2), 265-270. 
16. Toth, Z., Yan, X.X., Haftoglou, S., Ribak, C.E., Baram, T.Z., Seizure-induced neuronal injury: vulnerability to febrile seizures in an immature rat model. J. Neurosci., 1998, 18(11), 42854294.

17. Chen, K., Baram, T.Z., Soltesz, I., Febrile seizures in the developing brain result in persistent modification of neuronal excitability in limbic circuits. Nat. Med., 1999, 5(8), 888-894.

18. Dube, C., Chen, K., Eghbal-Ahmadi, M., Brunson, K., Soltesz, I., Baram, T.Z., Prolonged febrile seizures in the immature rat model enhance hippocampal excitability long term. Ann. Neurol., 2000, 47(3), 336-344.

19. Dube, C., et al., Temporal lobe epilepsy after experimental prolonged febrile seizures: prospective analysis. Brain, 2006, 129(4), 911-922.

20. Bender, R.A., Dube, C., Gonzalez-Vega, R., Mina, E.W., Baram, T.Z., Mossy fiber plasticity and enhanced hippocampal excitability, without hippocampal cell loss or altered neurogenesis, in an animal model of prolonged febrile seizures. Hippocampus, 2003, 13(3), 399-412.

21. Parent, J.M., Yu, T.W., Leibowitz, R.T., Geschwind, D.H., Sloviter, R.S., Lowenstein, D.H., Dentate granule cell neurogenesis is increased by seizures and contributes to aberrant network reorganization in the adult rat hippocampus. J. Neurosci., 1997, 17(10), 3727-3738.

22. Radley, J.J., Jacobs, B.L., Pilocarpine-induced status epilepticus increases cell proliferation in the dentate gyrus of adult rats via a 5-HT1A receptor-dependent mechanism. Brain Res., 2003, 966(1), 1-12.

23. Sankar, R., Shin, D., Liu, H., Katsumori, H., Wasterlain, C.G., Granule cell neurogenesis after status epilepticus in the immature rat brain. Epilepsia, 2000, 41(Suppl. 6), S53-56.

24. Gray WP, May K, Sundstrom LE., Seizure induced dentate neurogenesis does not diminish with age in rats. Neurosci. Lett., 2002, 330(3), 235-238.

25. Nakagawa, E., Aimi, Y., Yasuhara, O., et al., Enhancement of progenitor cell division in the dentate gyrus triggered by initial limbic seizures in rat models of epilepsy. Epilepsia, 2000, 41(1), 10-18.

26. Scott, B.W., Wang, S., Burnham, W.M., De Boni, U., Wojtowicz, J.M., Kindling-induced neurogenesis in the dentate gyrus of the rat. Neurosci. Lett.,1998, 248(2), 73-76.

27. Parent, J.M., Janumpalli, S., McNamara, J.O., Lowenstein, D.H., Increased dentate granule cell neurogenesis following amygdala kindling in the adult rat. Neurosci. Lett., 1998, 247(1), 912.

28. Tanapat, P., Hastings, N.B., Reeves, A.J., Gould, E., Estrogen stimulates a transient increase in the number of new neurons in the dentate gyrus of the adult female rat. J. Neurosci., 1999, 19(14), 5792-5801.

29. Perfilieva, E., Risedal, A., Nyberg, J., Johansson, B.B., Eriksson, P.S., Gender and strain influence on neurogenesis in dentate gyrus of young rats. J. Cereb. Blood Flow Metab., 2001 21(3), 211-217. 
30. Lennington, J.B, Yang, Z., Conover, J.C., Neural stem cells and the regulation of adult neurogenesis. Reprod. Biol. Endocrinol., 2003, 1(1), 99.

31. Mejias-Aponte, C.A., Jimenez-Rivera, C.A., Segarra, A.C., Sex differences in models of temporal lobe epilepsy: role of testosterone. Brain. Res., 2002, 944(1-2), 210-218.

32. Standley, C.A., Mason, B.A., Cotton, D.B., Differential regulation of seizure activity in the hippocampus of male and female rats. Am. J. Obstet. Gynecol., 1995, 173(4), 1160-1165.

33. Hayes, N.L., Nowakowski, R.S., Dynamics of cell proliferation in the adult dentate gyrus of two inbred strains of mice. Brain Res. Dev. Brain Res., 2002, 134(1-2), 77-85.

34. O'Keefe, J.A., Handa, R.J., Transient elevation of estrogen receptors in the neonatal rat hippocampus. Brain Res. Dev. Brain Res., 1990, 57(1), 119-127.

35. Pang, S.F., Caggiula, A.R., Gay, V.L., Goodman, R.L., Pang, C.S., Serum concentrations of testosterone, oestrogens, luteinizing hormone and follicle-stimulating hormone in male and female rats during the critical period of neural sexual differentiation. J. Endocrinol., 1979, 80(1), 103-110.

36. Seress, L., The effect of the neonatal testosterone treatment on the postnatal cell formation of the rat brain. Med. J. Osaka Univ., 1978, 28(3-4), 285-289.

37. Garcia-Segura, L.M., Azcoitia, I., DonCarlos, L.L., Neuroprotection by estradiol. Prog. Neurobiol., 2001, 63(1), 29-60.

38. Singer, C.A., Rogers, K.L., Strickland, T.M., Dorsa, D.M., Estrogen protects primary cortical neurons from glutamate toxicity. Neurosci. Lett., 1996, 212(1), 13-16.

39. Akman, C., Zhao, Q., Liu, X., Holmes, G.L., Effect of food deprivation during early development on cognition and neurogenesis in the rat. Epilepsy Behav., 2004, 5(4), 446-454.

40. Stern, J.M., Lonstein, J.S., Nursing behavior in rats is impaired in a small nestbox and with hyperthermic pups. Dev. Psychobiol., 1996, 29(2), 101-122.

41. Gould, E., Tanapat, P., Stress and hippocampal neurogenesis. Biol. Psychiatry, 1999, 46(11), 1472-1479.

42. Tanapat, P., Galea, L.A., Gould, E., Stress inhibits the proliferation of granule cell precursors in the developing dentate gyrus. Int. J. Dev. Neurosci., 1998, 16(3-4), 235-239.

43. de Kloet, E.R., Oitzl, M.S., Who cares for a stressed brain? The mother, the kid or both? Neurobiol. Aging, 2003, 24(Suppl. 1), S61-68.

44. Severino, G.S., Fossati, I.A., Padoin, M.J., et al., Effects of neonatal handling on the behavior and prolactin stress response in male and female rats at various ages and estrous cycle phases of females. Physiol. Behav., 2004, 81(3), 489-498.

45. Liu, H., Kaur, J., Dashtipour, K., Kinyamu, R., Ribak, C.E., Friedman, L.K., Suppression of hippocampal neurogenesis is associated with developmental stage, number of perinatal seizure episodes, and glucocorticosteroid level. Exp. Neurol., 2003, 184(1), 196-213.

46. Dube, C., Yu, H., Nalcioglu, O., Baram, T.Z., Serial MRI after experimental febrile seizures: Altered T2 signal without neuronal death. Ann. Neurol., 2004, 56(5), 709-714. 
47. Edwards, H.E., MacLusky, N.J., Burnham, W.M., The effect of seizures and kindling on reproductive hormones in the rat. Neurosci. Biobehav. Rev., 2000, 24(7), 753-762.

48. Morrell, M.J., Epilepsy in women: the science of why it is special. Neurology, 1999, 53(4 Suppl. 1), S42-48.

49. Porter, B.E., Maronski, M., Brooks-Kayal, A.R., Fate of newborn dentate granule cells after early life status epilepticus. Epilepsia, 2004, 45(1), 13-19.

50. McCabe, B.K., Silveira, D.C., Cilio, M.R., et al., Reduced neurogenesis after neonatal seizures. J. Neurosci., 2001, 21(6), 2094-2103.

51. Dayer, A.G., Ford, A.A., Cleaver, K.M., Yassaee, M., Cameron, H.A., Short-term and longterm survival of new neurons in the rat dentate gyrus. J. Comp. Neurol., 2003, 460(4), 563572.

52. Shapiro, L.A., Ribak, C.E., Integration of newly born dentate granule cells into adult brains: hypotheses based on normal and epileptic rodents. Brain Res. Brain Res. Rev., 2005, 48(1) 43-56. 


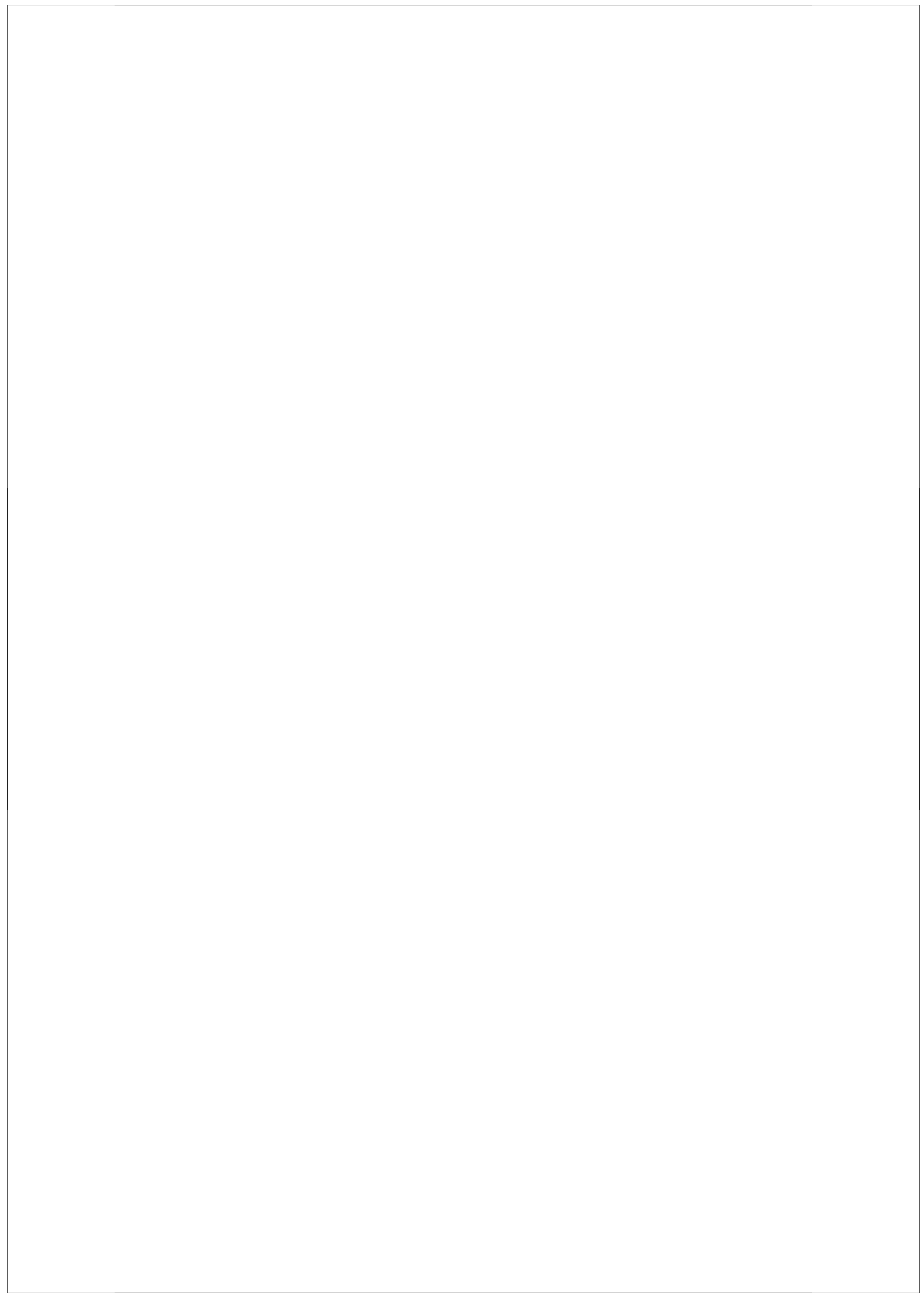



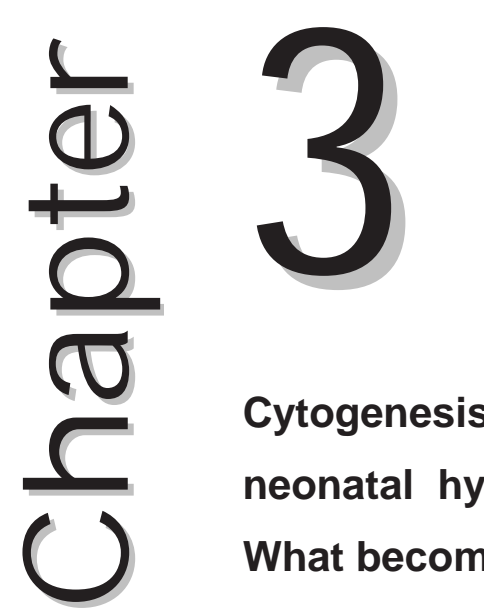

\section{Cytogenesis in the dentate gyrus after} neonatal hyperthermia-induced seizures: What becomes of surviving cells?

Evi M.P. Lemmens, Olaf E.M.G. Schijns,

Emile A.M. Beuls, Govert Hoogland

Epilepsia, 2008, 49 (5), 853-860.

ABSTRACT

Febrile seizures (FS) are early-life seizures thought to play a role in epileptogenesis. By labeling cells that were dividing immediately following experimental FS, we previously demonstrated that significantly more of these newborn cells in the dentate gyrus (DG) survived eight weeks later, relative to animals that did not experience FS. The purpose of the present study was to determine the long-term fate of these newborn cells.

On postnatal day (PN) 10 , hyperthermia-induced seizures $\left(\mathrm{HT}, \pm 42^{\circ} \mathrm{C}\right.$ core temperature) were evoked in SpragueDawley rats and littermates were used as normothermia controls (NT, $\pm 35^{\circ} \mathrm{C}$ core temperature). From PN11 to PN16, rats were injected with bromodeoxyuridine (BrdU) to label dividing cells. At PN66, we evaluated the number of BrdU-labeled cells in the DG that colocalized with the neuronal marker NeuN, glial marker glial fibrillary acidic protein (GFAP), neuronal excitatory amino acid transporter 3 (EAAT3), GABAergic neuronal marker glutamic acid decarboxylase 67 (GAD67) or microglia marker tomato lectin (TL).

In all rats, almost all BrdU-labeled cells in the DG, that showed double-labeling, colocalized with NeuN, and rarely with GFAP, GAD67, or TL. In NT controls and HT rats that did not experience seizures ('HT-no seizures'), 23\% of BrdU-labeled cells colocalized with EAAT3, which was significantly different from $14 \%$ in $\mathrm{HT}$ rats that did experience seizures $(\mathrm{HT}+\mathrm{FS})$.

Early-life seizures decrease the population of newborn cells that survive and mature into EAAT3-positive neurons and do not affect the GABAergic cell population. This may affect hippocampal physiology in young adulthood. 


\section{Introduction}

Febrile seizures (FS), which occur in association with a fever in children, are the most common type of seizures in young children. They are a topic of much research since it remains unclear whether FS have a causal relationship with the development of temporal lobe epilepsy (TLE) in young adults. Since FS occur in a critical period of brain development, characterized by neurogenesis, migration and differentiation, much research has focused on the consequences.

In the past, it has been suggested that epilepsy is due to an imbalance between excitability and inhibition leading to synchronization, which causes neurons to fire abnormally. Several hypotheses have been proposed to explain why a high fever in children leads to seizures [1-3] and epileptogenesis. A hypothesis that has gained much attention in recent years is that of seizure-induced proliferation of dentate granule cells (DGCs), the so-called 'neurogenesis hypothesis of epileptogenesis'. These newborn cells are thought to incorporate abnormally into the existing network in such a manner that they promote synchronization and hyperexcitability [4-6]. Seizures have been found to increase proliferation in adult animals $[7,8]$ and decrease $[9,10]$ or increase [11] proliferation in immature animals. Previously, we used a model of experimental hyperthermia(HT)-induced (febrile) seizures developed by Baram et al. [12] to study short- and long-term effects of experimental FS on proliferation in the DG. By labeling cells that were dividing immediately following FS with bromodeoxyuridine (BrdU), we demonstrated that two weeks after FS there was no change in the number of surviving BrdU-labeled newborn cells. Similar observations were made by Bender et al. [13]. However, eight weeks after FS, more BrdU-labeled cells had survived in rats that had experienced seizures than in normothermia control rats [14]. Because cellular densities were not changed, we suggested previously that FS may have caused some degree of cell death that is compensated for by incorporating these surviving cells into the network. This new population may affect the hippocampal physiology, and rats 
that had FS have been shown to develop spontaneous seizures later in life [15], it is therefore imperative to characterize the phenotype of these surviving cells.

A central question of FS-induced epileptogenesis is the temporal resolution at which a shift in the excitation/inhibition balance occurs and when the clinical and/or pathological signs of epilepsy (e.g. TLE) emerge. The clearance of neurotransmitters from the synaptic cleft is important in regulating excitability and inhibition. One group of transmembrane proteins, called the excitatory amino acid transporters (EAATs), is particularly responsible for maintaining low, non-toxic extracellular glutamate concentrations. In addition to two vesicular glutamate transporters (VGLUT1 and 2), there are five EAAT isoforms that are localized on the cell membrane of glial cells (EAAT1/GLAST and EAAT2/GLT) or neurons (EAAT3/EAAC1, EAAT4, and EAAT5; for review: [16]). Changes in the expression and localization of EAATs have been shown to be associated with seizures in several animal models [17-20] and in patients with TLE [21-23]. Because glutamic acid decarboxylase (GAD) converts glutamate to GABA, it is vital for the maintenance of the inhibitory tone exerted by GABAergic neurons (for review: [24]). Of the two GAD isoforms, GAD65 is mainly localized in the nerve terminals and GAD67 in the cell soma [25]. Patients with TLE were shown to have an increased expression of GAD [22]. In the animal model of experimental FS, no change in the total number of GAD67 expressing neurons could be found [13] when focusing on cell death in specific neuronal populations. Gliosis is one of the main features of HS, the pathological substrate found in the majority of TLE patients [26]. TLE patients with HS showed increased immunoreactivity for activated microglia [27] which indicates that HS is not an inert scar but is characterized by ongoing neuronal degeneration. Also in animal studies, seizures accompanied by cell death result in glial activation $[28,29]$.

The purpose of this study was to determine if hyperthermic seizures-induced proliferation could have a lasting effect on the constitution of the hippocampal neuronal network, thereby possibly contributing to a hyperexcitable state. 
Previously we labeled, with BrdU, cells that were born immediately after HTinduced seizures in immature male and female rats [14]. The current study represents a further analysis of the male population, in which we now characterized the phenotype of surviving BrdU-labeled cells in the DG by double-immunostaining with NeuN to label neurons, GFAP to label astrocytes, or tomato-lectin (TL) to label microglia. Our main goal was to analyze if the hyperexcitability, that was shown to occur after experimental FS [1-3] may be accompanied by a change in glutamate-related and/or GABA-related proteins and therefore double-labeled cells with BrdU and EAAT3 or GAD67. 


\section{Materials and M ethods}

\section{Animals}

The data described in this study have been obtained from the same animals as described previously [14]. Briefly, litters of six-day-old Sprague-Dawley rat pups were obtained from Harlan (Horst, The Netherlands). All rats were housed under standard conditions (i.e. $21 \pm 2^{\circ} \mathrm{C}$ ambient temperature, a 12-hour light/dark schedule, background noise provided by a radio, and food and water ad libitum). Sixteen rats were divided into the following three groups: (1) normothermia control rats $\left(\mathrm{NT}, \sim 35^{\circ} \mathrm{C}, \mathrm{n}=4\right.$ ), (2) hyperthermia rats that displayed behavioral seizures during hyperthermia exposure $\left(\mathrm{HT}+\mathrm{FS}, \sim 41^{\circ} \mathrm{C}\right.$, $\mathrm{n}=6$ ), and (3) hyperthermia rats that did not display behavioral seizures during hyperthermia exposure ('HT-no seizures', $\sim 41^{\circ} \mathrm{C}, \mathrm{n}=6$ ). The latter group was generated but was not analyzed in the previous study [14]. All experimental procedures were approved by the local ethical committee of Maastricht University and conformed to governmental policies.

\section{Hyperthermia induction}

HT was induced as described previously [12,14]. Briefly, on postnatal day (PN) 10 , rat pups were injected subcutaneously with $0.2 \mathrm{ml} 0.9 \%$ saline to prevent dehydration and placed in a Perspex cylinder with a diameter of $10 \mathrm{~cm}$ (one rat per cylinder). An adjustable stream of heated air, coming from a hair dryer placed $50 \mathrm{~cm}$ above the cylinder, was blown into the cylinder to raise the core temperature from about $35^{\circ} \mathrm{C}$ (normal temperature) to $\sim 42.5^{\circ} \mathrm{C}$. Core temperatures were measured rectally before and every $2.5 \mathrm{~min}$ during HT treatment. When the core temperature reached $39.5^{\circ} \mathrm{C}$, usually after $7 \mathrm{~min}$, the temperature and volume of the air were adjusted to maintain the core temperature at $41-42.5^{\circ} \mathrm{C}$ for $30 \mathrm{~min}$. If the core temperature exceeded $42.5^{\circ} \mathrm{C}$, the rat was removed from the cylinder until its temperature dropped to $40-42.5^{\circ} \mathrm{C}$. The occurrence of seizures was monitored behaviorally by two 
observers independently. Behavioral seizures were stereotyped and previously shown to correlate with EEG discharges in the hippocampus [3,12]. Immediately after the 30 min treatment the rats were cooled in room temperature (RT) water and then returned to their mother. NT controls were exposed to the same conditions except that the stream of air was used to maintain the normal core temperature of the rats. At PN21, all pups were weaned and randomly housed two to three per cage.

\section{BrdU labeling and tissue processing}

From PN11-16, all rats received twice daily (minimum six hours apart) intraperitoneal injections of the thymidine analog 5'bromo-2'deoxyuridine (BrdU, $25 \mathrm{mg} / \mathrm{kg}$, Sigma, St. Louis, MO, USA), $2 \mathrm{mg} / \mathrm{ml}$ in $0.9 \%$ saline (pH 7.6). At PN66, the rats received an overdose of Nembutal $₫$, followed by perfusion fixation with $4 \%$ paraformaldehyde in $0.1 \mathrm{M}$ phosphate buffer (PB, pH 7.6). After the brains were removed, they were postfixated in $4 \%$ paraformaldehyde/ $0.1 \mathrm{M}$ PB for $48 \mathrm{~h}\left(4^{\circ} \mathrm{C}\right)$, and cryoprotected in $20 \%$ sucrose/0.1 M PB for $\left(24 \mathrm{~h}, 4^{\circ} \mathrm{C}\right)$. Coronal serial sections ( $10 \mu \mathrm{m}$; between Bregma -1.8 and $+5.5 \mathrm{~mm}$ ) were cut using a cryostat and mounted on Superfrost $₫$ slides.

\section{BrdU detection}

For BrdU detection, the sections were washed in TBS $(0.1 \mathrm{M}$ Tris-base, $0.15 \mathrm{M}$ $\mathrm{NaCl}, \mathrm{pH}$ 7.4), treated with TBS containing $0.3 \% \mathrm{H}_{2} \mathrm{O}_{2}$ for $30 \mathrm{~min}$, and washed again in TBS $\left(2 \times 10 \mathrm{~min}\right.$ at $\mathrm{RT}, 1 \times 10 \mathrm{~min}$ at $\left.65^{\circ} \mathrm{C}\right)$. For DNA denaturation, the sections were incubated in $50 \%$ formamide/2x SSC buffer $(0.3 \mathrm{M} \mathrm{NaCl}, 0.03 \mathrm{M}$ sodium citrate; $\mathrm{pH} 7.0$ ) for $2 \mathrm{~h}$ at $65^{\circ} \mathrm{C}$, rinsed in $2 x \mathrm{SSC}$ buffer (RT), incubated in $2 \mathrm{~N} \mathrm{HCl}$ for $30 \mathrm{~min}$ at $37^{\circ} \mathrm{C}$, and incubated in $0.1 \mathrm{M}$ borate buffer $(\mathrm{pH} 8.5)$ for $10 \mathrm{~min}(\mathrm{RT})$. After this pretreatment, the sections were washed six times in TBS, incubated in TBS-TS (TBS containing $0.25 \%$ Triton X-100 and $3 \%$ normal donkey serum) for $60 \mathrm{~min}(\mathrm{RT})$, and then incubated overnight in primary antiBrdU antibody (monoclonal mouse, 1:800 in TBS-TS; Roche, the Netherlands). 
After rinsing the sections in TBS, they were incubated for $1 \mathrm{~h}$ in secondary biotinylated donkey anti-mouse antibody (Jackson Immunoresearch Laboratories, West Grove, PA, USA; 1:400 in TBS-TS). The staining was visualized with a Vectastain $\mathrm{ABC} /$ Elite standard kit (Vector Laboratories, Burlingame, CA, USA), based on the avidin-biotin-peroxidase reaction, with diaminobenzidine as chromogen, and $\mathrm{NiCl}_{2}$ as a signal enhancer. Finally, the sections were counterstained with $0.2 \%$ cresyl violet for $1 \mathrm{~h}$, dehydrated, and coverslipped with DePeX. Sections of the NT and HT groups were processed simultaneously to minimize inter-assay variability.

\section{Immunofluorescent double-labeling}

The sections were pretreated the same as for $\mathrm{BrdU}$ detection, except that the sections were not incubated with $\mathrm{H}_{2} \mathrm{O}_{2}$. For double-labeling of BrdU and EAAT3 or GAD67, the sections were incubated in $0.01 \mathrm{M}$ sodium citrate buffer $(2 \mathrm{x}$ $5 \mathrm{~min}$ at $95^{\circ} \mathrm{C}$ ) before the incubation in $50 \%$ formamide. Primary antibodies used for double-labeling were all diluted in TBS-TS and included anti-BrdU (mouse monoclonal, 1:400; Roche) and either anti-NeuN (mouse monoclonal, biotinylated, 1:50; Chemicon, CA, USA), anti-GFAP (rabbit polyclonal, 1:2,000; Dako Cytomation, Belgium), anti-TL (biotinylated, 1:100; Vector), anti-GAD67 (polyclonal rabbit, 1:100; Santa Cruz biotechnology, CA, USA), or anti-EAAT3 (rabbit polyclonal, 1:100; Alpha Diagnostics Intl., TX, USA). After overnight incubation (RT), the sections were washed with TBS three times for $10 \mathrm{~min}$ each. The sections were then incubated for $60 \mathrm{~min}$ (RT) with a secondary antibody to detect BrdU (biotinylated donkey anti-mouse, 1:400 in TBS-TS; Jackson Immunoresearch Laboratories), rinsed with TBS for three times $10 \mathrm{~min}$ each, and incubated with streptavidin conjugated to Alexa 594 (Invitrogen, Breda, the Netherlands) diluted 1:2,000 in TBS (90 min, RT). Next, GFAP, EAAT3, and GAD67 were stained by incubating the sections with donkey antirabbit antibody conjugated to Alexa 488 (Invitrogen) diluted 1:100 in TBS

containing $0.25 \%$ Triton X-100 (120 min, RT). NeuN and TL were stained using 
biotinylated donkey anti-mouse (1:400 in TBS-TS; Jackson Immunoresearch Laboratories) followed by washing in TBS ( $3 \times 10 \mathrm{~min})$ and incubation in streptavidin-Alexa488 (Invitrogen) diluted 1:1,000 in TBS (90 min, RT). Finally, all sections were rinsed in TBS $(4 \times 10 \mathrm{~min})$ and coverslipped using TBS/glycerol (20\%/80\%).

\section{Quantitative analysis}

The analysis of the BrdU staining was conducted using a computerized stereology workstation, consisting of a modified light microscope (Olympus BX51; Olympus, Tokyo, Japan), a motorized specimen stage for automatic sampling, a CCD color video camera, and stereology software (Stereolnvestigator; MicroBrightField Inc., Williston, VT, USA). For BrdU quantification, six sections per rat were used. In each section the entire DGC layer (DGL), visualized by cresyl violet counterstaining, was delineated by hand using the coordinates from the stereotaxic atlas [30]. BrdU-positive cell counts were estimated using the Fractionator probe [31]. All cells that came into focus within unbiased virtual counting frames ( 40 by $40 \mu \mathrm{m}$; within a grid of 100 by $100 \mu \mathrm{m}$ ) were counted. Our criterion for counting an individual cell was the presence of its nucleus either within the counting frame or touching the right or top frame lines (green) but not touching the left or bottom lines (red). Thin $(10 \mu \mathrm{m})$ sections were used that minimized staining gradients due to incomplete penetration of the antibody, and by which cells on top of each other were very rarely seen. The number of BrdU-positive cells per 100,000 $\mu^{2}{ }^{2}$ was calculated, and as we did not find a significant difference in surface area or cell density between the groups, we expressed the cell counts of the HT groups as percentage of NT (mean set at $100 \%$ ). Cell counts were performed by two observers blinded to the treatment-status of the sections, and showed no significant inter-observer variation.

Double-stained sections were evaluated using a Nikon F50 fluorescence microscope that was connected to a computer with image-based analysis 
software (AnalySIS $®$ Soft Imaging System GmbH, Münster, Germany). Per section, 50 randomly chosen BrdU-labeled cells in the DGL were analyzed for double-labeling with NeuN, GFAP, TL, GAD67 or EAAT3. The number of BrdU/EAAT3 labeled cells was expressed as a percentage of the $50 \mathrm{BrdU}$ positive cells that were evaluated for double-labeling. A sample of cells that were identified as double-labeling using conventional fluorescence microscopy, were also evaluated using confocal microscopy which confirmed their doublelabeling in all cases.

\section{Statistical analysis}

HT rats were compared with NT control rats using analysis of variance (ANOVA) combined with LSD or Bonferroni's multiple comparison post hoc test, using 'Statistical Package for the Social Sciences' (SPSS) 11.5 (significance level set at $p<0.05)$. 


\section{Results}

The HT treatment differently affected rat behavior: $65 \%$ of the rats displayed behavioral seizures $(\mathrm{HT}+\mathrm{FS})$ that were typically characterized by arrest of hyperkinesias, followed by falling on their side or back with body flexion and clonic contractions of the limbs, whereas $35 \%$ of the rats did not display this stereotyped behavior and were therefore denoted as 'HT-no seizures'. None of the NT rats showed abnormal behavior during their stay in the cylinder.
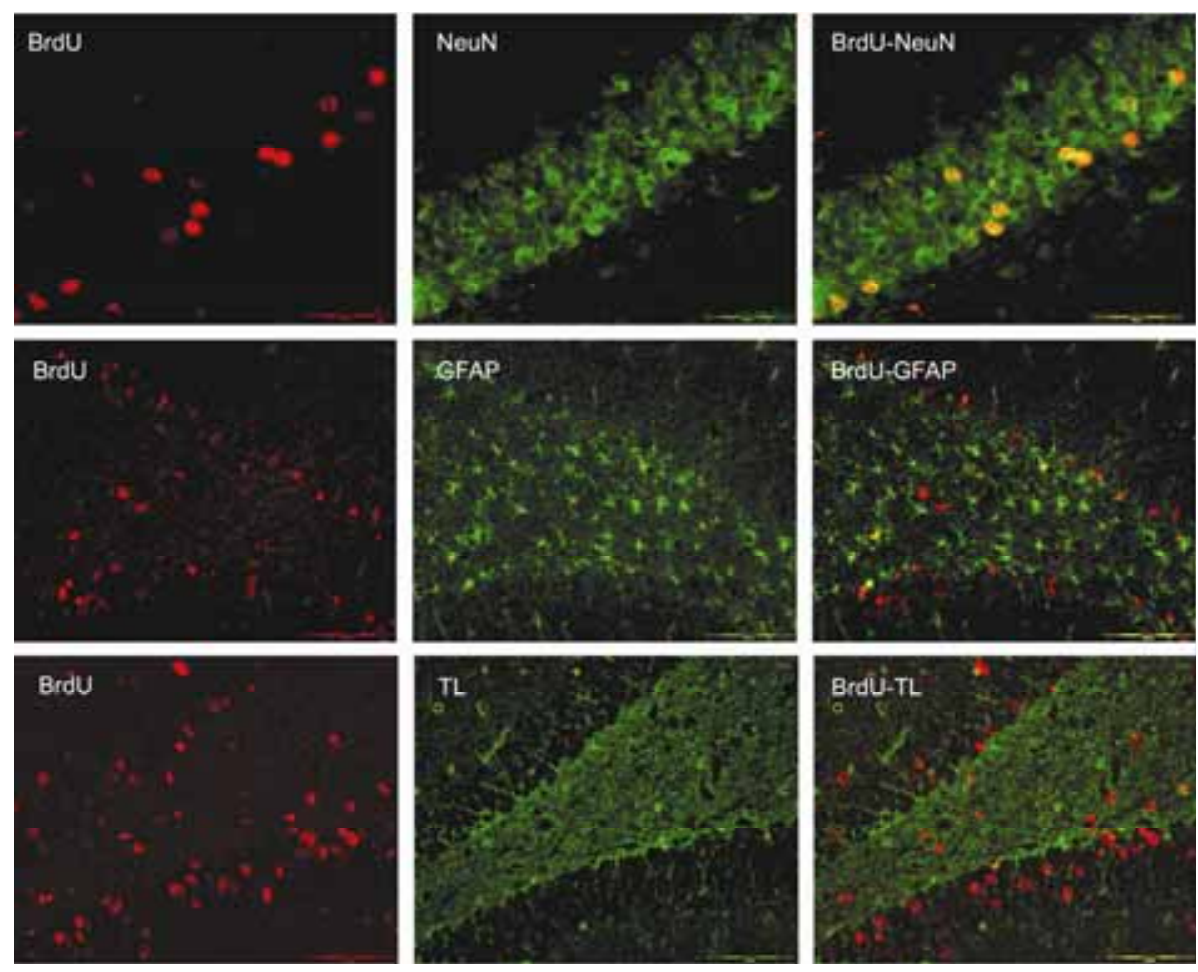

FIG. 1. BrdU-NeuN/GFAP/TL (tomato lectin) double-labeling. Typical example of a hippocampal section of a NT rat, stained with anti-BrdU (red) and anti-NeuN, anti-GFAP, or anti-TL antibodies (green). Almost all BrdU-positive cells colocalized with NeuN (yellow-orange). There was no difference in the number of cells that were immunopositive for BrdU that colocalized with GFAP or with TL in any experimental group. Scale bars: top images $(\mathrm{BrdU}-\mathrm{NeuN})=50 \mu \mathrm{m}$, bottom images $(\mathrm{BrdU}-\mathrm{GFAP} / \mathrm{TL})=100 \mu \mathrm{m}$. 

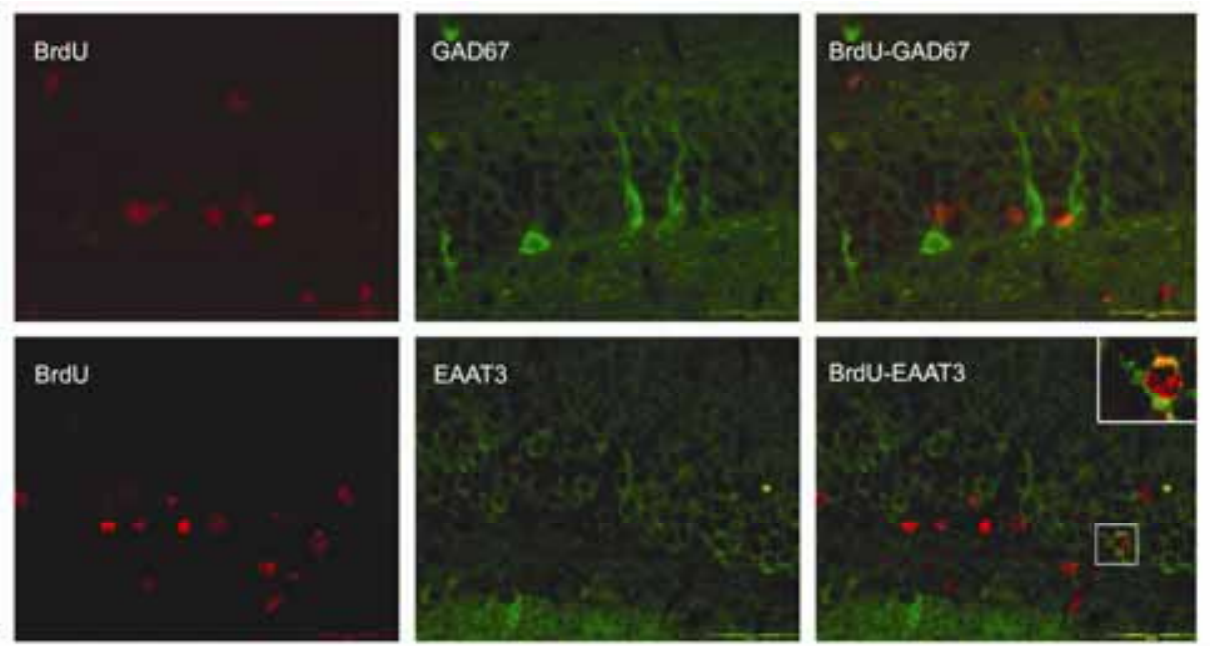

FIG. 2. BrdU-GAD67/EAAT3 double-labeling. Pictures of the DGC layer of a control rat. Top: Only a small number of cells in the rat dentate gyrus (DG) were GAD67-positive (green). None of the newborn cells, immunopositive for BrdU (red), colocalized with GAD67. These results were similar in NT controls, 'HT-no seizures' rats, and HT+FS rats. Bottom: several BrdU-positive cells did colocalize with EAAT3 (see detail in box). Here, the cell nucleus is stained in red (BrdU) while the cell cytoplasm is positive for EAAT3 (green). Scale bars $=50 \mu \mathrm{m}$.

Using the same experimental design as described in this study, i.e. analysis of BrdU-positive DG cells eight weeks after HT treatment, we previously found $25 \%$ more cells in HT+FS (125 $\pm 5 \%$ ) than in NT rats (100 $\pm 7 \%)$ [14]. However, it was not known whether this increase was due to the HT treatment or to the $\mathrm{HT}$-induced seizures. Analysis of the number of newborn cells in the 'HT-no seizures' group (106 $\pm 9 \%$ ) now demonstrates that this group exhibits a similar number of BrdU-positive cells as NT rats $(p$-value $=0.61$ ) and had less newborn cells than $\mathrm{HT}+\mathrm{FS}$ rats, though not statistically significant $(\mathrm{p}$-value $=0.07)$.

To determine the phenotype of the surviving newborn cells in the DG, we characterized BrdU-positive cells by double-labeling with NeuN, GFAP, TL, GAD67, and EAAT3. The results showed that BrdU almost exclusively colocalized with NeuN, and scarcely with GFAP or TL (fig. 1). Only a small number of BrdU-positive cells in the hilus colocalized with GFAP and some 
BrdU-positive cells at the border of the molecular layer with the DGL colocalized with TL and had the morphological appearance of endothelial cells with a flattened cell body and nucleus (data not shown). No differences in the staining pattern of BrdU/NeuN, BrdU/GFAP or BrdU/TL, between NT, 'HT-no seizures' and $\mathrm{HT}+\mathrm{FS}$ rats, were seen.

Newborn cells that developed into GABAergic cells were identified by doublelabeling BrdU with GAD67. This analysis showed that in either rat group only a few cells in the DG were GAD67-positive, but none of them colocalized with BrdU. In contrast to GAD67, about $23 \%$ of the BrdU-positive cells colocalized with EAAT3 in NT and 'HT-no seizures' rats (fig. 2). This number dropped significantly to $14 \%$ in $H T+F S$ rats $\left(F_{2,12}=7,681 ; p<0.01\right)$ (fig. 3$)$.

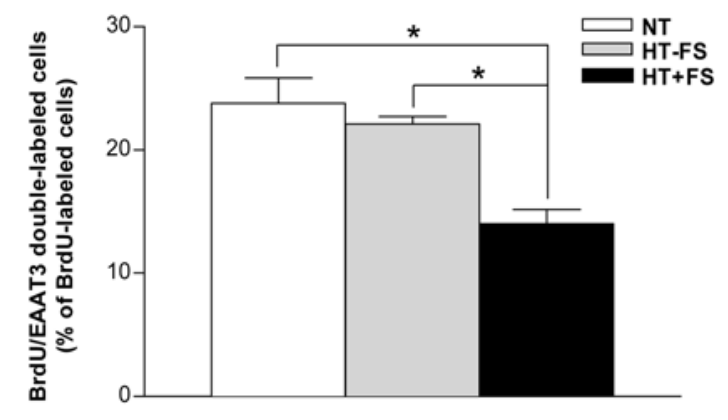

FIG. 3. Quantification of BrdU/EAAT3 double-labeling. Quantification of the double-labeling showed that the amount of double-labeled cells in $\mathrm{HT}+\mathrm{FS}$ rats $(n=6)$ dropped to about $14 \%$ compared to control $(n=4)$ and 'HT-no seizures (HT-FS)' rats $(n=5)$ that had about $23 \%$ of co-localizing cells $\left({ }^{*} p<0.01\right)$. Data are presented as mean + SEM. 


\section{Discussion}

Clinical as well as experimental data suggest that early-life seizures increase the risk of developing epilepsy in later life, but the molecular mechanisms underlying this relationship are not well understood. It has been postulated that early-life seizures distort the neuronal development and thereby contribute to epileptogenesis. In the present study we used an immature rat model, labeled cells that were born immediately following HT-induced seizures, and studied their phenotype in adulthood. The findings of this study are: (a) in control, as well as in animals that had seizures, nearly all newborn cells developed into mature NeuN-positive neurons and not into GFAP-positive astrocytes or TLpositive microglial cells, (b) HT-induced seizures, but not HT, significantly increased the number of BrdU-labeled cells and reduced the number of newborn cells that survived and matured into EAAT3-expressing cells, and (c) the number of GAD67-positive GABAergic cells was not affected by HT or seizures.

\section{HT-induced seizures at PN10 do not cause astrogliosis}

Febrile seizures have often been associated with the development of hippocampal sclerosis-associated TLE. Reactive gliosis is one of the histopathological hallmarks of hippocampal sclerosis, so in order to examine if HT-induced seizures can cause gliosis, we analyzed whether cells that are after born HT-induced seizures develop into an astroglial, a microglial or a neuronal phenotype. Although the astrocytic marker GFAP did stain hilar cells, very few of them co-labeled with BrdU and none of the cells were BrdU/GFAP positive. Using the same experimental design, with the exception of BrdU labeling, we previously quantified the GFAP immunoreactivity of different hippocampal subfields by optical density and found no difference in mean grey value between HT+FS, 'HT-no seizures', and NT rats at 2 weeks and 8 weeks postseizure (unpublished observations). Ravizza et al. [28] showed an increased 
GFAP expression after kainic acid-induced status epilepticus in animals at PN15 and PN21, but not at PN9, similar to our results. Co-labeling of BrdU with TL, a marker for microglial cells (and endothelial cells/blood vessels; [32]), also showed a very small amount of BrdU/TL-positive cells. Similar results were found in other early-life seizure models [10]. For instance, kainic acid-induced seizures cause little activation of microglia and astrocytes at PN9, but significant glial activation and cell damage at PN21 [29]. To further validate the absence of gliosis following HT-induced seizures, we investigated whether the newborn cells had developed into mature neurons. Nearly all BrdU-positive cells that were found eight weeks after HT-induced seizures colocalized with NeuN. Similar results were found in other early-life seizures models. For instance, Porter et al. [33] showed that almost $95 \%$ of cells born after lithium-pilocarpineinduced status epilepticus developed into NeuN-positive neurons. Also in the flurothyl-induced seizure model, McCabe et al. [10] found that $93 \%$ of the BrdUimmunoreactive cells colocalized with NeuN. Thus, HT seizure-induced cytogenesis results in the generation of mature neurons and does not contribute to gliosis in the animal model studied here.

\section{HT-induced seizures have a long-lasting effect on dentate granule cell layer composition}

Previously, we found $25 \%$ more BrdU-labeled cells in the DG of adult male rats that had experienced HT-induced seizures in early-life [14], and we now identified these BrdU-labeled cells as neurons. As described before, HT does not always induce behavioral seizures at PN10 [12]. In our hands, about $65 \%$ of the HT treated rats also displayed behavioral seizures. Since we do not have electrographic data to support these findings, we cannot exclude that 'HT-no seizures' rats may have experienced subclinical seizures during or after the hyperthermia treatment. Interestingly, the number of BrdU positive cells that was found in the HT-no seizures group resembles more that of the normothermia controls than that of the HT+FS group. The current study 
therefore suggests that the increased survival of BrdU-labeled cells is a seizure specific effect. Similarly, it was recently shown that early-life, pilocarpineinduced seizures result in an increased survival of BrdU-labeled cells in the DG [34].

Since it has been shown that HT-induced seizures cause persistent hyperexcitability of the hippocampus $[1,35]$ and spontaneous seizures in about $35 \%$ of the adult animals [15], our purpose was to determine if the newborn cells developed an excitatory or inhibitory phenotype. This was done by doublelabeling newborn cells with GAD67 or EAAT3. We found no evidence of BrdU/GAD67 colocalization and GAD-positive cells were rarely seen in the dentate gyrus. Although GAD expression can be inhibited by FS in rat pups, this was only found in pups 2-5 days old, not in pups of 10-15 days old as we used in our experiments [36]. In other models of seizures, such as the pilocarpine model, GAD expression (both GAD65 and 67) has been found to be increased after seizures [37]. This has been observed mainly in hippocampal areas that are vulnerable to seizure-induced damage. Since the animal model for FS used in our experiments is characterized by only minor and transient damage [38,39], it is not surprising that GAD expression was not found to be elevated 8 weeks after FS.

In contrast to the absence of BrdU-GAD67 colocalization, $23 \%$ of all BrdUpositive cells colocalized with EAAT3 in control rats. In HT+FS rats, the percentage of BrdU/EAAT3 labeled cells was reduced to $14 \%$. As EAAT3 functions to eliminate glutamate from the synaptic cleft, the expression of EAAT3 might influence excitability.

Although the functional consequences of the changes in glutamate transporter expression after $\mathrm{HT}$-induced seizures found in the present study are unclear, it has been shown that a reduction of EAAT3 expression by injecting anti-sense oligonucleotide probes led to seizures [40]. A reduction of EAAT3/EAAC1 may not only lead to a decreased glutamate clearance and hyperexcitability at the glutamatergic synapse, but may also affect GABAergic synapses. Because 
glutamate serves as a precursor for GABA synthesis it is possible that a decreased glutamate uptake causes even more hyperexcitability by decreasing GABA levels. Such a correlation between GABA levels and EAAC1 expression has already been demonstrated [19].

To conclude, we can only infer the possible consequences the decreased population of newborn cells expressing EAAT3 might have, and more research is needed to elucidate the results of our findings on hippocampal excitability. However, it is possible that the results reflect another mechanism of hyperexcitability leading to hyperthermia-induced epileptogenesis. 


\section{References}

1. Chen, K., et al., Persistently modified h-channels after complex febrile seizures convert the seizure-induced enhancement of inhibition to hyperexcitability. Nat. Med., 2001, 7(3), 331337.

2. Chen, K., Baram, T.Z., Soltesz, I., Febrile seizures in the developing brain result in persistent modification of neuronal excitability in limbic circuits. Nat. Med., 1999, 5(8), 888-894.

3. Dube, C., et al., Prolonged febrile seizures in the immature rat model enhance hippocampal excitability long term. Ann. Neurol., 2000, 47(3), 336-344.

4. Parent, J.M., The role of seizure-induced neurogenesis in epileptogenesis and brain repair. Epilepsy Res., 2002, 50(1-2), 179-189.

5. Scharfman, H.E., Goodman, J.H., Sollas, A.L., Granule-like neurons at the hilar/CA3 border after status epilepticus and their synchrony with area CA3 pyramidal cells: functional implications of seizure-induced neurogenesis. J. Neurosci., 2000, 20(16), 6144-6158.

6. Shapiro, L.A., Ribak, C.E., Integration of newly born dentate granule cells into adult brains: hypotheses based on normal and epileptic rodents. Brain Res. Brain Res. Rev., 2005, 48(1), 43-56.

7. Parent, J.M., et al., Increased dentate granule cell neurogenesis following amygdala kindling in the adult rat. Neurosci. Lett., 1998, 247(1), 9-12.

8. Parent, J.M., et al., Dentate granule cell neurogenesis is increased by seizures and contributes to aberrant network reorganization in the adult rat hippocampus. J. Neurosci., 1997, 17(10), 3727-3738.

9. Liu, H., et al., Suppression of hippocampal neurogenesis is associated with developmental stage, number of perinatal seizure episodes, and glucocorticosteroid level. Exp. Neurol., 2003, 184(1), 196-213.

10. McCabe, B.K., et al., Reduced neurogenesis after neonatal seizures. J. Neurosci., 2001, 21(6), 2094-2103.

11. Sankar, R., et al., Granule cell neurogenesis after status epilepticus in the immature rat brain. Epilepsia, 2000, 41(Suppl. 6), S53-56.

12. Baram, T.Z., Gerth, A., Schultz, L., Febrile seizures: an appropriate-aged model suitable for long-term studies. Brain Res. Dev. Brain Res., 1997, 98(2), 265-270.

13. Bender, R.A., et al., Mossy fiber plasticity and enhanced hippocampal excitability, without hippocampal cell loss or altered neurogenesis, in an animal model of prolonged febrile seizures. Hippocampus, 2003, 13(3), 399-412.

14. Lemmens, E.M., et al., Gender differences in febrile seizure-induced proliferation and survival in the rat dentate gyrus. Epilepsia, 2005, 46(10), 1603-1612.

15. Dube, C., et al., Temporal lobe epilepsy after experimental prolonged febrile seizures: prospective analysis. Brain, 2006, 129(4), 911-922. 
16. Danbolt, N.C., Glutamate uptake. Prog. Neurobiol., 2001, 65(1), 1-105.

17. Alonso-Nanclares, L., De Felipe, J., Vesicular glutamate transporter 1 immunostaining in the normal and epileptic human cerebral cortex. Neuroscience, 2005, 134(1), 59-68.

18. Furuta, A., et al., Translocation of glutamate transporter subtype excitatory amino acid carrier 1 protein in kainic acid-induced rat epilepsy. Am. J. Pathol., 2003, 163(2), 779-787.

19. Sepkuty, J.P., et al., A neuronal glutamate transporter contributes to neurotransmitter GABA synthesis and epilepsy. J. Neurosci., 2002, 22(15), 6372-6379.

20. Ueda, Y., et al., Collapse of extracellular glutamate regulation during epileptogenesis: downregulation and functional failure of glutamate transporter function in rats with chronic seizures induced by kainic acid. J. Neurochem., 2001, 76(3), 892-900.

21. Crino, P.B., et al., Increased expression of the neuronal glutamate transporter (EAAT3/EAAC1) in hippocampal and neocortical epilepsy. Epilepsia, 2002, 43(3), 211-218.

22. Mathern, G.W., et al., Hippocampal GABA and glutamate transporter immunoreactivity in patients with temporal lobe epilepsy. Neurology, 1999, 52(3), 453-472.

23. Proper, E.A., et al., Distribution of glutamate transporters in the hippocampus of patients with pharmaco-resistant temporal lobe epilepsy. Brain, 2002, 125(1), 32-43.

24. Erlander, M.G., Tobin, A.J., The structural and functional heterogeneity of glutamic acid decarboxylase: a review. Neurochem. Res., 1991, 16(3), 215-226.

25. Esclapez, M., et al., Comparative localization of two forms of glutamic acid decarboxylase and their mRNAs in rat brain supports the concept of functional differences between the forms. $J$. Neurosci., 1994, 14(3/2), 1834-1855.

26. Blumcke, I., Thom, M., Wiestler, O.D., Ammon's horn sclerosis: a maldevelopmental disorder associated with temporal lobe epilepsy. Brain Pathol., 2002, 12(2), 199-211.

27. Beach, T.G., et al., Reactive microglia in hippocampal sclerosis associated with human temporal lobe epilepsy. Neurosci. Lett., 1995, 191(1-2), 27-30.

28. Ravizza, T., et al., Inflammatory response and glia activation in developing rat hippocampus after status epilepticus. Epilepsia, 2005, 46(Suppl. 5), 113-117.

29. Rizzi, M., et al., Glia activation and cytokine increase in rat hippocampus by kainic acidinduced status epilepticus during postnatal development. Neurobiol. Dis., 2003, 14(3), 494503.

30. Paxinos, G., Watson, C., The Rat Brain in Stereotaxic Coordinates. 4th ed. 1998, San Diego, CA: Academic Press.

31. Gundersen, H.J., et al., The new stereological tools: disector, fractionator, nucleator and point sampled intercepts and their use in pathological research and diagnosis. Apmis, 1988, 96(10), 857-881.

32. Acarin, L., et al., Demonstration of poly-N-acetyl lactosamine residues in ameboid and ramified microglial cells in rat brain by tomato lectin binding. J. Histochem. Cytochem., 1994, 42(8), 1033-1041. 
33. Porter, B.E., Maronski, M., Brooks-Kayal, A.R., Fate of newborn dentate granule cells after early life status epilepticus. Epilepsia, 2004, 45(1), 13-19.

34. Xiu-Yu, S., Ruo-Peng, S., Ji-Wen, W., Consequences of pilocarpine-induced recurrent seizures in neonatal rats. Brain Dev., 2007, 29, 157-163.

35. Tsai, M.L., Leung, L.S., Decrease of Hippocampal GABA Receptor-Mediated Inhibition after Hyperthermia-induced Seizures in Immature Rats. Epilepsia, 2006, 47(2), 277-287.

36. Arias, C., Valero, H., Tapia, R., Inhibition of brain glutamate decarboxylase activity is related to febrile seizures in rat pups. J. Neurochem., 1992, 58(1), 369-373.

37. Esclapez, M., Houser, C.R., Up-regulation of GAD65 and GAD67 in remaining hippocampal GABA neurons in a model of temporal lobe epilepsy. J. Comp. Neurol., 1999, 412(3), 488505.

38. Dube, C., et al., Serial MRI after experimental febrile seizures: Altered T2 signal without neuronal death. Ann. Neurol., 2004, 56(5), 709-714.

39. Toth, Z., et al., Seizure-induced neuronal injury: vulnerability to febrile seizures in an immature rat model. J. Neurosci., 1998, 18(11), 4285-4294.

40. Rothstein, J.D., et al., Knockout of glutamate transporters reveals a major role for astroglial transport in excitotoxicity and clearance of glutamate. Neuron, 1996, 16(3), 675-686. 


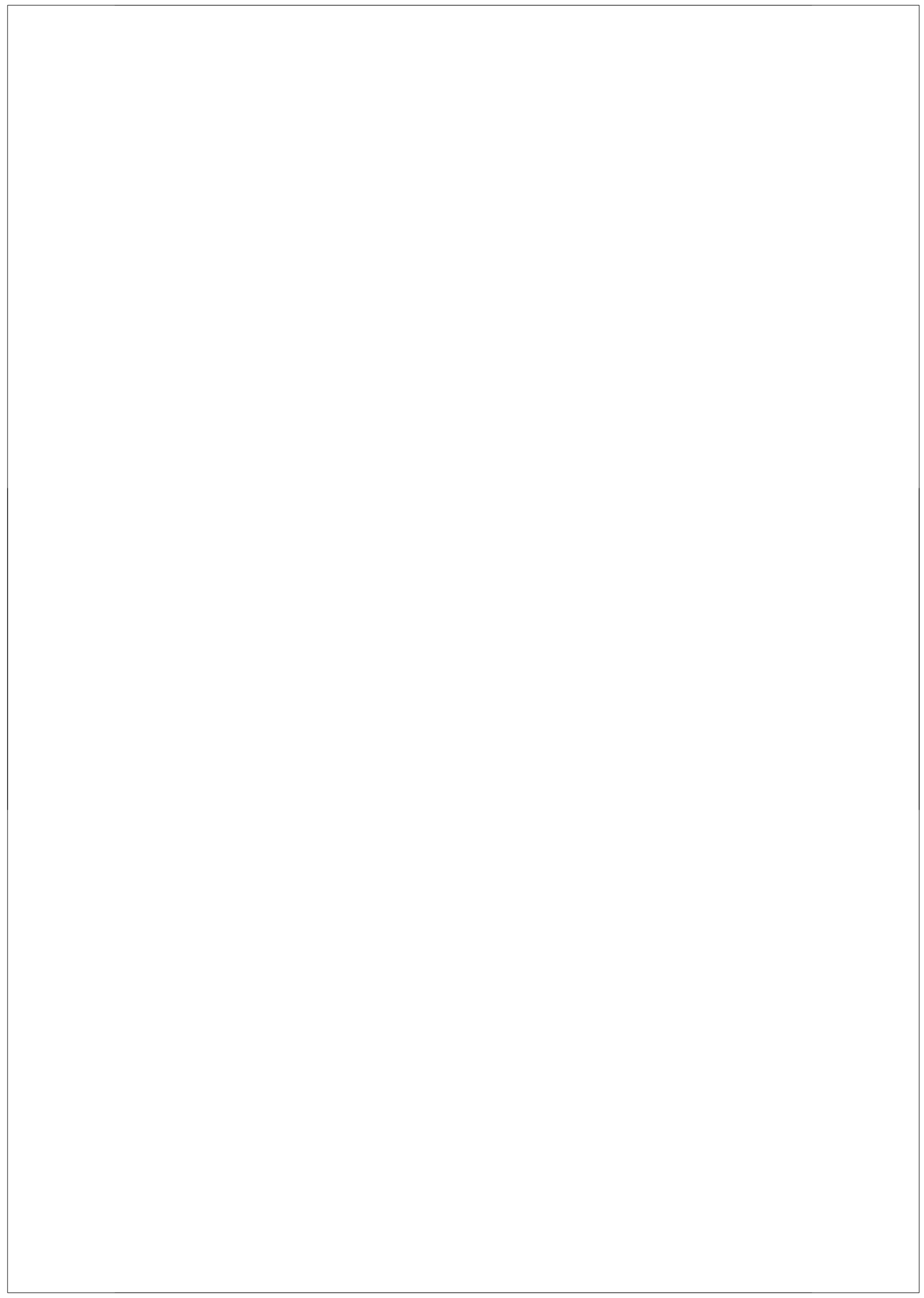



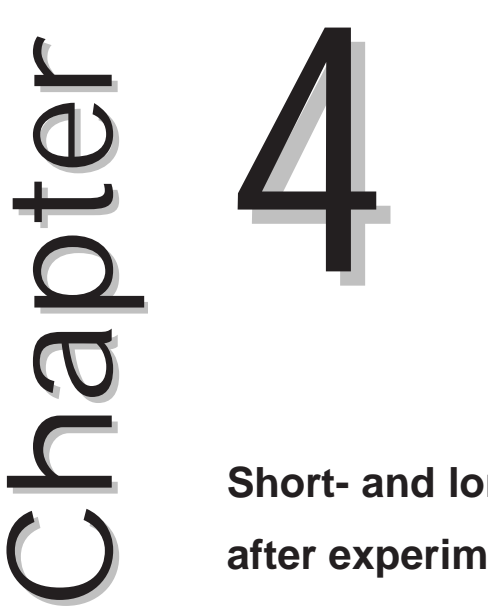

Short- and long- term limbic abnormalities after experimental febrile seizures

Jacobus F.A. Jansen*, Evi M.P. Lemmens*, Gustav J. Strijkers,

Jeanine J. Prompers, Olaf E.M.G. Schijns, M. Eline Kooi, Emile A.M. Beuls, Klaas Nicolay, Walter H. Backes, Govert Hoogland

*Authors contributed equally

Neurobiology of Disease, 2008, July 27 (online early).

ABSTRACT

Experimental febrile seizures (FS) are known to promote hyperexcitability of the limbic system and increase the risk for eventual temporal lobe epilepsy (TLE). Early markers of accompanying microstructural and metabolic changes may be provided by in vivo serial MRI.

FS were induced in 9-day old rats by hyperthermia. Quantitative multimodal MRI was applied 24 hours and 8 weeks later, and comprised hippocampal volumetry and proton spectroscopy, and cerebral T2 relaxometry and diffusion tensor imaging (DTI). At 9 weeks histology was performed.

Hippocampal T2 relaxation time elevations appeared to be transient. DTI abnormalities detected in the amygdala persisted up to 8 weeks. Hippocampal volumes were not affected, while developmental MR changes in $\mathrm{N}$-acetylaspartate and diffusion anisotropy were observed. Histology showed increased fiber density and anisotropy in the hippocampus, and reduced neuronal surface area in the amygdala.

Quantitative serial MRI is able to detect long-term FS-induced microstructural alterations. These findings support the concept that FS are related to TLE. 


\section{Introduction}

The impact of febrile seizures (FS) on neurodevelopment is still largely unknown. Though they are generally considered benign, case reports, epidemiological, and retrospective studies suggest that a subpopulation of patients is at risk for developing mesial temporal sclerosis-associated temporal lobe epilepsy (MTS-TLE) [1-3]. Diagnostic tools that can identify this subpopulation before they clinically manifest epilepsy are necessary to develop a rational anticipatory treatment. Because MTS-TLE patients are characterized by structural and metabolic abnormalities of the limbic system, it may be possible to visualize the development of these abnormalities by serial magnetic resonance imaging (MRI). MRI has several important beneficial features. First, quantitative MRI can image and quantify disease related changes over time [4]. Second, its versatile capabilities allow investigation of distinct tissue characteristics, including microstructural ( $\mathrm{T} 2$ relaxation time and diffusion tensor imaging (DTI)) and metabolic (proton spectroscopy) changes.

Longitudinal MRI studies addressing the potential causative association between FS and MTS-TLE are scarce and have primarily utilized hippocampal T2 and volumetric measurements. These studies presented cases with a unilateral increase in hippocampal T2 signal and volume within days after prolonged FS. A follow-up MRI 2-10 months later demonstrated normal or increased hippocampal T2 relaxation time and reduced volume [5-8]. It was therefore suggested that FS occasionally cause acute hippocampal edema that evolves into sclerosis. However, hippocampal sclerosis (HS, i.e. elevated T2 relaxation time and reduced volume) was not observed in all patients that showed post-FS edema. This raises the questions whether the follow-up MRI had been acquired too early and whether a possible developing MTS would have been noticed with other MR modalities. A severe difficulty in prospective studies is the long and variable latent period that may exist between FS and 
eventual HS allowing the occurrence of other factors (e.g. trauma, seizures, or infection) that potentially bias the outcome.

Animal models may prove to be valuable in prospective MRI as they allow a more standardized experimental design and conditions [9]. Moreover, animal models open the possibility for comparing in vivo imaging data with postmortem histology. A recent T2 weighted MRI study has demonstrated early effects, i.e. $87 \%$ of rats with prolonged FS at postnatal day (PN) 11 had increased T2 signal in the limbic region 8 days after the seizure [10]. Interestingly, the T2 abnormalities were not accompanied by histological evidence of neurodegeneration. A subsequent study showed that $35 \%$ of the FS rats had spontaneous electro-clinical seizures and $88 \%$ displayed interictal epileptiform discharges at PN180 [11]. The present study examined this model using quantitative multimodal MRI and serially (24 hours and 8 weeks after FS) assessed the brains of FS-induced rats. Histological analysis was performed 9 weeks after FS.

The aim of this study was to determine early and late microstructural and metabolic changes induced by experimental FS, which are possibly related to pathologic cellular processes linked to epileptogenesis. Revealing cellular processes underlying these changes may provide insights in how early-life FS, tissue abnormalities, and (late) epilepsy are related. 


\section{Materials and Methods}

\section{Hyperthermia treatment}

Sprague-Dawley rats (Harlan, The Netherlands) were born and housed under standard conditions. Experiments were approved by the local Animal Experiments Committee.

Hyperthermia (HT) was induced as described previously [12,13]. In brief, on $\mathrm{PN9}$, rat pups $(\mathrm{HT}+)$ were placed in a cylinder, their core temperature raised with an adjustable stream of heated air to $41-42.5^{\circ} \mathrm{C}$ for 30 minutes. Core temperatures were measured before and every $2.5 \mathrm{~min}$ during the HT treatment. The behavioral seizures were stereotyped, and consisted of arrest of heat-induced hyperkinesia, followed by body flexion, and occasionally followed by clonic contractions of the limbs, and were previously shown to correlate with hippocampal EEG discharges [12,14]. Littermates were used as normothermia (NT) controls, meaning that they were exposed to the same conditions, except that the stream of heated air was used to maintain the innate core temperature $\left(35^{\circ} \mathrm{C}\right)$ of the rats. At PN21, all pups were weaned and randomly housed, 2-3 rats per cage.

\section{Sample size estimation}

Dubé et al. [10] used the same animal model and observed changes in T2weighted signal intensities in the hippocampus 8 days after the hyperthermia treatment of $37 \%$, with a standard deviation of $27 \%$, compared to control rats. A power calculation [15] based on these results by Dubé et al., shows that a sample size of 18 rats ( $2 \times 9$ rats per group) is required to reach a statistical power of $80 \%$ (i.e. $20 \%$ risk of type II errors) at a significance level of $\alpha=0.05$. 


\section{MR}

MR experiments were performed on a 6.3 Tesla magnet (Oxford Instruments, England) interfaced to a Bruker Biospec console (Bruker, Ettlingen, Germany), using a linear transmit volume coil and a butterfly surface receive coil (Rapid Biomed, Rimpar, Germany). MR was performed 24 hours after HT (PN10) on $9 \mathrm{NT}$ and $11 \mathrm{HT}+$ rats, and 8 weeks later (PN66) on $9 \mathrm{NT}$ and $9 \mathrm{HT}+$ rats. Anesthesia was induced with a mixture of $2-4 \%$ isoflurane and medicinal air (a carrier gas), and maintained with 1-2.5\% isoflurane. For anatomical reference, a proton density and T2-weighted multi slice multi spin-echo pulse sequence (MSME) was used acquiring 15 coronal slices $(1 \mathrm{~mm}$ ) with a repetition time (TR) of $4937 \mathrm{~ms}$ and echo times (TE) of 12.2 and $128.3 \mathrm{~ms}$ ( $256 \times 192$ matrix, field of view $4 \times 4 \mathrm{~cm}^{2}, 1$ average, duration 12 minutes). Quantitative T2 imaging was performed using an MSME sequence with a TR of $5 \mathrm{~s}$ and TE: 17.2, 43.0, 77.3, 111.7, 146.1, and $180.4 \mathrm{~ms}$ ( 15 coronal slices, $1 \mathrm{~mm}, 128 \times 128$ matrix, field of view $4 \times 4 \mathrm{~cm}^{2}, 2$ averages, duration 8 minutes). T2 relaxation times were calculated on a pixel-by-pixel basis using a nonlinear monoexponential fit. For $\mathrm{DTI}$, an echo planar imaging sequence was used, with 30 directions ( $T R=3 \mathrm{~s}$, $\mathrm{TE}=34 \mathrm{~ms}, \mathrm{~b}=0$ and $1000 \mathrm{~s} / \mathrm{mm}^{2}$, diffusion gradient duration $4 \mathrm{~ms}$, diffusion gradient strength $239.1 \mathrm{mT} / \mathrm{m}, 15$ coronal slices, $1 \mathrm{~mm}, 128 \times 128$ matrix, field of view $4 \times 4 \mathrm{~cm}^{2}, 2$ averages, duration 14 minutes). The apparent diffusion coefficient (ADC, unit $10^{-6} \mathrm{~mm}^{2} / \mathrm{s}$ ) and fractional anisotropy (FA, unit \%) maps were calculated on a voxel-by-voxel basis. Single-voxel ${ }^{1} \mathrm{H}-\mathrm{MRS}$ was applied to a $5 \times 4 \times 2 \mathrm{~mm}^{3}(0.04 \mathrm{ml})$ voxel mainly covering the bilateral hippocampi, but also partially the retrosplenial corti, thalamus and corpus callosum (fig. 1A). Single voxel spectroscopy was performed, rather than spectroscopic imaging, as spectra obtained using spectroscopic imaging would not have allowed reliable quantification of the neurotransmitters GABA and glutamate. The following parameters were used: TE $14 \mathrm{~ms}$, TR $10 \mathrm{~s}, 256$ averages, spectral bandwidth $4006.41 \mathrm{~Hz}$, and number of points 1977. Localization and water suppression were achieved with point-resolved spatially localized spectroscopy (PRESS) 
and chemical shift selective suppression (CHESS), respectively. The total preparation time and acquisition time of the spectroscopy procedure was approximately 1 hour. For absolute quantification of metabolite concentrations expressed in $\mathrm{mmol} / \mathrm{l}$, the calibration strategy based on the water reference signal was used [16]. To this end, after the in vivo measurement, the signal from unsuppressed tissue water was recorded from the same voxel, which served as an endogenous concentration reference. The unsuppressed spectrum was recorded under identical conditions (16 averages) as the metabolite spectra, but with the water-suppression radiofrequency pulses switched off. Relaxation correction was performed utilizing onsite determined T2 and T1 relaxation parameters for water (averaged per group; data not shown), and previously reported relaxation times for metabolites by de Graaf et al [17]. Absolute concentrations were determined using previously reported values of water content by Tkac et al [18].

\section{MR data analysis}

Image processing and analysis was performed using the software package MRIcro [19] and software in Matlab (The Mathworks, Natick, MA, USA). Hippocampal volumetry was performed by two independent observers blinded to the treatment status of the animals using images from the MSME sequence with TE $=128.3 \mathrm{~ms}$. The agreement between observers was calculated using the Pearson correlation coefficient and coefficient of variation. Further analysis was based on values averaged over both observers. Furthermore, on the T2weighted coronal slice with coordinates Bregma $-3.30 \mathrm{~mm}$ and interaural $5.70 \mathrm{~mm}$, bilateral regions of interest (ROI) were manually drawn within the thalamus, hippocampus, retrosplenial cortex, amygdala, piriform cortex, and corpus callosum (fig. 1B), conform the Paxinos brain atlas [20]. The mean T2, $A D C$ and $F A$ values were calculated for the selected structures.

The spectra were analyzed using the LCModel software package (Version 6.14), which analyzes the in vivo MR spectra as a linear combination of the 
separately recorded in vitro spectra of the individual metabolites. The metabolite basis set (PRESS, TE $14 \mathrm{~ms}, 6.3 \mathrm{~T}$ ) including simulated macromolecule peaks was kindly provided by Dr. Provencher. For each spectrum, the parts per million (ppm) range included for analysis was 0.2-4.2 ppm. LCModel provides estimates in $\mathrm{mmol} / \mathrm{kg}$ for a total of 16 metabolites, including $\mathrm{N}$-acetyl-aspartate (NAA), choline (Cho), total creatine (tCr), myo-inositol $(\mathrm{ml})$, taurine (Tau), and the neurotransmitters glutamate (Glu) and gamma-aminobutyric acid (GABA). The Cramer-Rao minimum variance was calculated as an estimate of the error in metabolite quantification [21]. Metabolite estimates were excluded from analysis, if the Cramer-Rao minimum variance exceeded the $20 \%$ range.

\section{Histological staining}

At PN73, 9 weeks after $\mathrm{HT}$, each rat $(\mathrm{NT}, \mathrm{n}=9 ; \mathrm{HT}+, \mathrm{n}=9)$ was transcardially perfused with $4 \%$ paraformaldehyde under pentobarbital anesthesia, the brains cryoprotected in $30 \%$ sucrose and coronal serial sections $(30 \mu \mathrm{m})$ cut using a cryostat [13].

One series of sections was Nissl stained using $0.1 \%$ cresylviolet. Another series of sections was stained with $0.2 \%$ Black-Gold II [22]. Black-Gold staining permits visualization of all myelinated fiber tracts which are all likely to influence FA values as derived by MRI, whereas Timms-staining [23] would only highlight mossy fibers. A third series of sections was immunohistochemically stained for glial fibrillary acidic protein (GFAP) using a monoclonal mouse primary antibody (1:2,000; Sigma, St. Louis, MO, USA), and biotinylated, donkey anti-mouse secondary antibody (Jackson Immunoresearch Laboratories, West Grove, PA, USA). Staining was visualized using diaminobenzidine. All three series of sections contained samples from every rat, and for each staining sections of control and FS rats were processed simultaneously to minimize interstaining variability. A negative control for the Black-Gold staining was made by omitting the step of Black-Gold incubation, and for the GFAP staining by omitting the primary antibody. 


\section{Histological analysis}

Hippocampal volume, amygdalar cell and hippocampal dentate granule cell (DGC) volume and density were quantified using the Nissl stained sections, and astrocyte density in the amygdala using the GFAP stained sections. All stereological analyses were carried out using a computerized stereology workstation, using the Stereolnvestigator software (MicroBrightField Inc., Williston, VT, U.S.A.). The volume of the hippocampus was calculated using the Cavalieri's principle, by delineating the region in 12-16 sections per animal (between Bregma -1.8 and -6.8 mm [20]). Cell density was measured using the Optical Fractionator, and neuronal volume using the Nucleator [24].

Black-Gold stained sections were used for analysis of fiber density. Stained sections were analyzed using an Olympus AX70 microscope using bright field illumination. Pictures were made with an F-view cooled CCD camera (Olympus, the Netherlands) and analyzed using ImageJ software (National Institute of Health (NIH), USA). An upper and lower threshold value, to distinguish background from specific staining, was determined using the negative control and the section with the darkest staining pattern, and was kept constant for all the measurements. Area fraction occupied by the stained fibers (as the percentage of pixels with a grey value within the threshold limits) was calculated by the ImageJ software. One section was used to analyze staining of the total hippocampus and amygdala (Bregma $-3.8 \mathrm{~mm}$ ), and three sections were used and averaged for the hippocampal granule cell layer (DGL) and hilus (Bregma 3.3/-4.3/-5.3 mm). Delineation of the ROls was done using the Stereotaxic Atlas [20].

Additionally, to quantify the directionality of fibers within the DGL in the hippocampus, a two-dimensional microscopic fractional anisotropy (MFA) analysis was performed using cross-correlation filter-diagonalization of local image intensities [25].

Histological analyses were performed blinded to the animal treatment status. 


\section{Statistical analysis}

Hippocampal volumetry, tissue T2, ADC, and FA of the right and left hemisphere were first compared for each $\mathrm{ROI}$ in each animal separately. A paired two-tailed Student's t-test revealed no significant differences between hemispheres. Data from the two hemispheres were therefore averaged for further analysis.

Statistical analysis was performed in two ways: (i) a rigid analysis, using correction for multiple comparisons (of multi-modal MR outcome values) to reveal which brain regions were affected by HT-treatment, and (ii) an analysis per modality, indicating which trends are present. Both types of analysis are presented as (i) yields firm, reliable statistical results based on any changes, and (ii) offers more insight in the nature and direction of possible changes. Note that regions with a significant effect, as determined by method (i) not necessarily have to yield a significant effect as determined by method (ii), or vice versa.

(i) Multiple end point testing was controlled for by first investigating in what regions MR-detectable $\mathrm{HT}$-induced alterations appeared. To this end, T2, ADC, and FA MRI outcomes were combined per ROI. It is important to note that these measures are substantially affected by changes in water content and combining these measures may provide much stronger sensitivity to tissue water changes than each individual measure. For each ROI, the global null hypothesis stating that within that region no differences between the HT+ and NT group of the included MR modalities exist was tested using the ordinary least squares test of O-Brien and Läuter $[26,27]$. For the combined analysis, statistical significance was calculated with two-tailed Student's t-tests. Hochberg correction was applied to compensate for multiple comparisons [28].

(ii) In a subsequent analysis per MR modality, hippocampal volumetry, tissue T2, ADC, FA values, and spectroscopic data of HT+ rats were compared with those of control rats at PN10 and PN66. To examine possible effects on 
maturation, the differences between the values at the two time points (PN66PN10) were also analyzed. For the separate modality and histology analyses, statistical significance was calculated with two-tailed Student's t-tests. For all statistical analyses, $p<0.05$ was considered significant. Data are expressed as mean \pm standard error of the mean. 

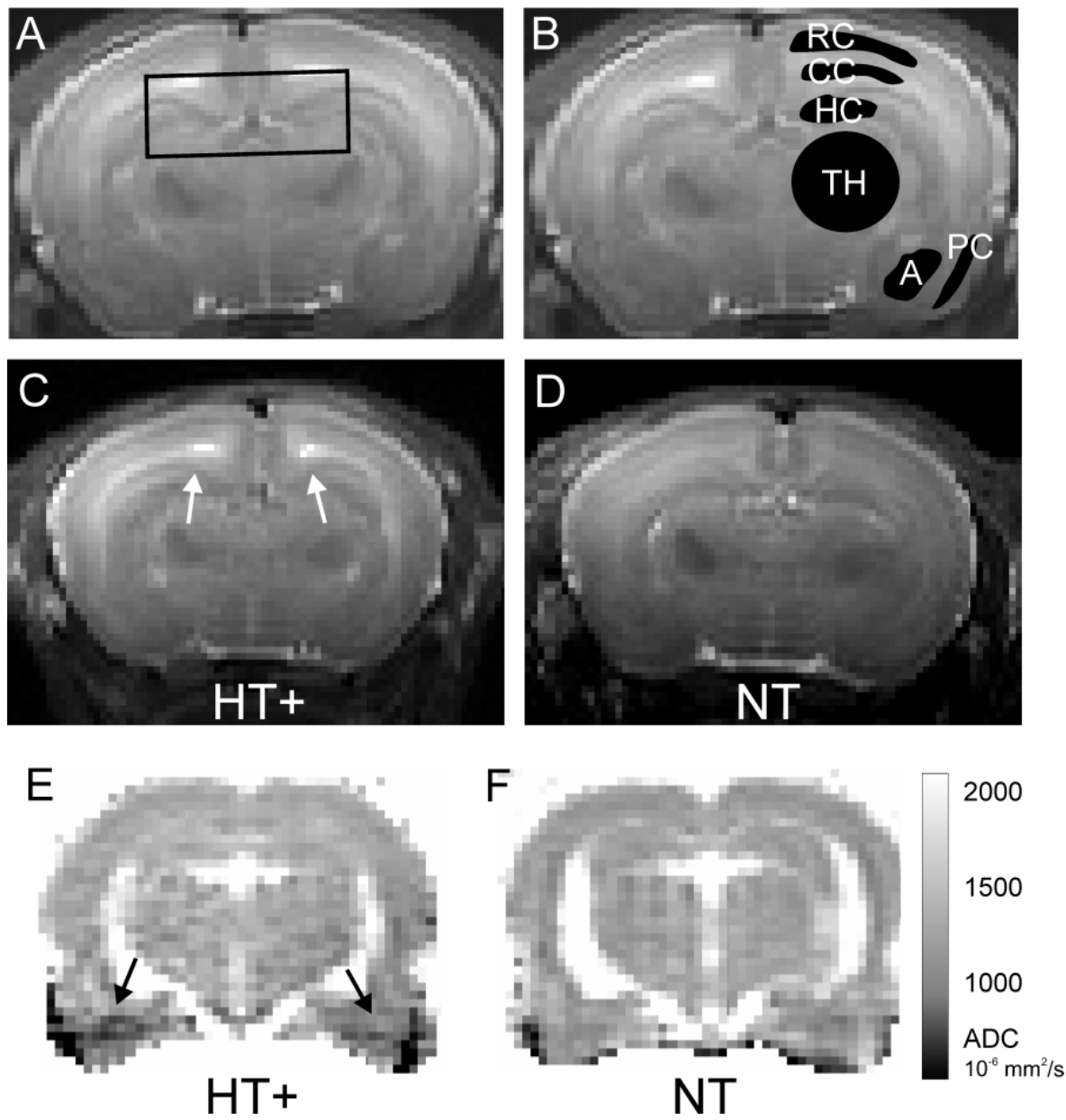

FIG. 1. MR images of NT and HT+ rats. T2-weighted image of a hyperthermia treated rat at PN10, indicating the voxel of interest for proton spectroscopy $(A)$, and the employed regions of interests for MRI analysis (B). Abbreviations: $T H$, thalamus; $H C$, hippocampus; RC, retrosplenial cortex; $A$, amygdala; PC, piriform cortex; and CC, corpus callosum. T2-weighted images 24 hours after treatment of a (C) hyperthermia (HT+) and (D) normothermia (NT) rat. Note the hyperintense MRI signal (white arrows) due to prolongation of the tissue T2 relaxation time, indicative of edema formation in (C) proximal to the corpus callosum and hippocampus of the $\mathrm{HT}+$ rat with compared to the NT rat (D). ADC maps at PN66 of the brain of a representative hyperthermia $(H T+)(E)$ and a normothermia (NT) treated rat $(F)$. Notice for the $H T+$ rat the pronounced ventral ADC decrease in the bilateral amygdala region (black arrows), which is indicative of chronic micro-structural tissue damage. 


\section{Results}

\section{Animal model}

Sixty-seven percent of the rats showed FS behavior after HT (HT+ rats) with a seizure duration of $9.1 \pm 2.0$ minutes. Mortality during the follow up MR examinations at PN66 was 18\% (2/11) and 0\% (0/9) for the HT+ group and normothermia group, respectively, and was probably due to respiratory failure during anesthesia. During the MR experiments, all control and experimental animals were stable, displaying normal body temperature and respiratory signal.

\section{RI findings}

COMBINED REGIONAL ANALYSIS. The ordinary least squares test revealed significant HT-induced MR alterations at PN10 for the retrosplenial cortex, corpus callosum, amygdala and piriform cortex and at PN66 for the hippocampus (table 1).

HIPPOCAMPAL VOLUMETRY. There were no statistical significant differences in hippocampal volumes on T2-weighted images between the two groups at either time points (table 1).

T2 RELAXOMETRY. At PN10, HT+ rats had elevated T2 relaxation time values in the hippocampus compared to NT rats (increase of $5 \%, p<0.05$ ). In figure $1 C D$, typical examples of T2-weighted MR images of rat brains at PN10 are shown, displaying an abnormal hyperintense signal due to T2 relaxation time prolongation in the region near the corpus callosum and hippocampus in the $\mathrm{HT}+$ rat. At PN66, no differences were found in T2 relaxation times between the groups (table 1).

DIFFUSION TENSOR IMAGING. ADC values were significantly decreased at PN10 (decrease of 10\%) for HT+ rats in the amygdala $(p<0.01)$ and piriform cortex $(p<0.05)$. At PN66 ADC values were decreased in the amygdala 
$(p<0.05)$. Figure 1EF displays an ADC map at PN66 of a typical HT+ and NT rat. For the $\mathrm{HT}+$ rat a decrease in $\mathrm{ADC}$ values can be appreciated ventrally.

\begin{tabular}{|c|c|c|c|c|c|c|c|}
\hline \multirow{2}{*}{\multicolumn{2}{|c|}{ Region of Interest }} & \multicolumn{3}{|c|}{ PN10 } & \multicolumn{3}{|c|}{ PN66 } \\
\hline & & \multirow{2}{*}{$\begin{array}{c}\mathrm{HT}+ \\
\text { Mean (SEM) }\end{array}$} & \multirow{2}{*}{\multicolumn{2}{|c|}{$\begin{array}{c}\text { NT } \\
\text { Mean (SEM) }\end{array}$}} & \multirow{2}{*}{$\begin{array}{c}\mathrm{HT}+ \\
\text { Mean (SEM) }\end{array}$} & \multirow{2}{*}{\multicolumn{2}{|c|}{$\begin{array}{c}\text { NT } \\
\text { Mean (SEM) }\end{array}$}} \\
\hline & Quantity & & & & & & \\
\hline \multicolumn{8}{|c|}{ Hippocampus } \\
\hline & T2 & $106.5(1.9) \dagger$ & & $101.3(1.6)$ & $53.4(2.3)-$ & $49.6(2.2)$ & \\
\hline & $A D C$ & $993(11)$ & & $1020(14)$ & $1240(62)$ & $\neq 1292(39)$ & \\
\hline & $F A$ & $19(1)$ & & $21(1)$ & $34(3)$ & $28(3)$ & * \\
\hline & Volume & $60(2)$ & & $64(2)$ & $114(6)$ & $115(3)$ & \\
\hline & $N A A$ & $1.7(0.2)$ & & $2.3(0.3)$ & $4.7(0.2)$ & $4.5(0.2)$ & * \\
\hline & $t C r$ & $5.2(1.0)$ & & $5.6(0.6)$ & $7.2(0.2) \dagger$ & $6.7(0.2)$ & \\
\hline & Cho & $1.1(0.2)$ & & $1.3(0.2)$ & $1.1(0.0)$ & $1.1(0.1)$ & \\
\hline & $G A B A$ & $2.2(0.2)$ & & $2.3(0.2)$ & $2.1(0.1)$ & $2.4(0.1)$ & \\
\hline & Glu & $3.6(0.3)$ & & $4.1(0.3)$ & $6.2(0.2)$ & $6.2(0.4)$ & \\
\hline & Tau & $9.4(1.0)$ & & $11.5(1.1)$ & $5.9(0.1)$ & $5.5(0.2)$ & \\
\hline & Ins & $3.7(0.8)$ & & $2.7(0.3)$ & $5.6(0.1)$ & $5.4(0.2)$ & \\
\hline \multicolumn{8}{|c|}{ Retrosplenial cortex } \\
\hline & T2 & $113.9(2.6)-$ & & $108.7(2.2)$ & $51.7(2.5)$ & $48.9(2.6)$ & \\
\hline & $A D C$ & $946(15)$ & $\neq$ & $977(15)$ & $1180(71)$ & $1239(47)$ & \\
\hline & $F A$ & $20(1)$ & & $21(1)$ & $34(3)$ & $30(3)$ & \\
\hline \multicolumn{8}{|c|}{ Corpus Callosum } \\
\hline & T2 & $120.9(4.0)-$ & & $118.2(2.3)$ & $49.8(1.6)$ & $47.4(1.6)$ & \\
\hline & $A D C$ & $1075(13)$ & $\ddagger$ & $1093(29)$ & $1227(62)$ & $1294(31)$ & \\
\hline & $F A$ & $24(1)$ & & $25(1)$ & $37(2)$ & $39(3)$ & \\
\hline \multicolumn{8}{|l|}{ Thalamus } \\
\hline & T2 & $95.9(0.9)$ & & $93.3(1.7)$ & $48.1(0.9)$ & $47.8(0.8)$ & \\
\hline & $A D C$ & $970(11)$ & & $1002(16)$ & $1170(55)$ & $1250(48)$ & \\
\hline & $F A$ & $20(1)$ & & $22(1)$ & $39(3) \dagger$ & $31(2)$ & \\
\hline \multicolumn{8}{|l|}{ Amygdala } \\
\hline & T2 & $84.5(1.3)$ & & $85.0(1.4)$ & $55.4(1.3)$ & $55.1(1.4)$ & \\
\hline & $A D C$ & $914(6) \dagger$ & $\ddagger$ & $962(11)$ & $1163(48) \dagger$ & $1320(39)$ & \\
\hline & $F A$ & $15(1)$ & & $16(1)$ & $39(3)$ & $32(2)$ & \\
\hline \multicolumn{8}{|c|}{ Piriform cortex } \\
\hline & T2 & $80.9(2.6)-$ & & $85.0(1.4)$ & $56.3(2.2)$ & $53.6(1.7)$ & \\
\hline & $A D C$ & $924(10) \dagger$ & $\ddagger$ & $972(15)$ & $1136(50)$ & $1217(45)$ & \\
\hline & $F A$ & $19(1)$ & & $17(1)$ & $38(3)$ & $35(2)$ & \\
\hline
\end{tabular}

TABLE 1. Quantitative MR results in hyperthermia and control rats.

$\mathrm{HT}+$, hyperthermia group; NT, normothermia control group; PN10, postnatal day 10; PN66, postnatal day 66; SEM, standard error of the mean; T2, transverse relaxation time (in ms); ADC, apparent diffusion coefficient (in $10^{-6} \mathrm{~mm}^{2} / \mathrm{s}$ ); FA, fractional anisotropy (in \%); Volume, hippocampal volume (in $\mu \mathrm{l}$ ); NAA, N-acetyl-aspartate; tCr, total creatine; Cho, choline; GABA, gammaaminobutyric acid; Glu, glutamate; tau, taurine; Ins, myo-inositol (all in mmol/kg wet weight) † 2-tailed $\mathrm{p}<0.05$

$\ddagger 2$-tailed $p<0.05$ (ordinary least squares test using Hochberg correction)

* 2-tailed $p<0.05$ time course maturation effect (PN66-PN10) (see fig. 2) 
For FA, no differences were observed at PN10. However, at PN66 FA values were higher $(+30 \%)$ in the thalamus $(p<0.05)$ (table 1$)$. The analysis of a possible HT-induced effect on maturation, revealed a significantly larger FA increase in the hippocampus between PN10 and PN66 (fig. 2A).
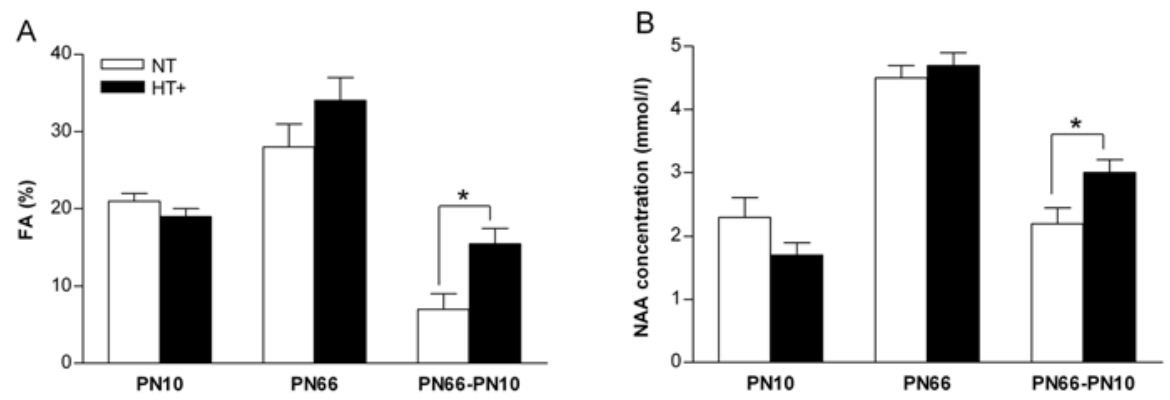

FIG. 2. Time course of maturation effects within the hippocampus. (A) Bar diagrams displaying the FA values within the hippocampus, for the hyperthermia treated ( $\mathrm{HT}+$, black bars) and normothermia treated (NT, white bars) rats, at PN10, PN66 and the difference PN66-PN10. (B) Bar diagrams displaying the concentration of $\mathrm{N}$-acetylaspartate (NAA) within the hippocampus, for the $\mathrm{HT}+$ (black bars), and NT (white bars) rats, at PN10, PN66 and the difference PN66-PN10. Bars represent mean value \pm standard error of the mean. ${ }^{*}$ denotes $p<0.05$.

OBSERVER AGREEMENT. The Pearson correlation coefficients between the data obtained by the two observers were $0.91,0.96,0.87$, and 0.75 and the coefficients of variation were $10 \%, 5 \%, 4 \%$, and $13 \%$, for the hippocampal volumetry, T2, ADC, and FA values, respectively.

SPECTROSCOPY. Figure 3 illustrates typical hippocampal proton spectra of a $\mathrm{HT}+$ rat at PN10 (fig. 3A) and PN66 (fig. 3B). While no changes were observed in the concentrations of the neurotransmitters glutamate and GABA, a trend towards a lower concentration of the neuronal marker NAA (decrease of $40 \%$ ) was observed at PN10 $(p<0.10)$. Also, a significantly higher concentration of $\mathrm{tCr}$ (increase of $7 \%$ ) was observed at PN66 for the HT+ rats $(p<0.05)$. Furthermore, the rate of NAA increase (to adult values) between PN10 and PN66 was significantly higher in the $H T+$ rats $(p<0.05)$ (fig. $2 B$ ). 

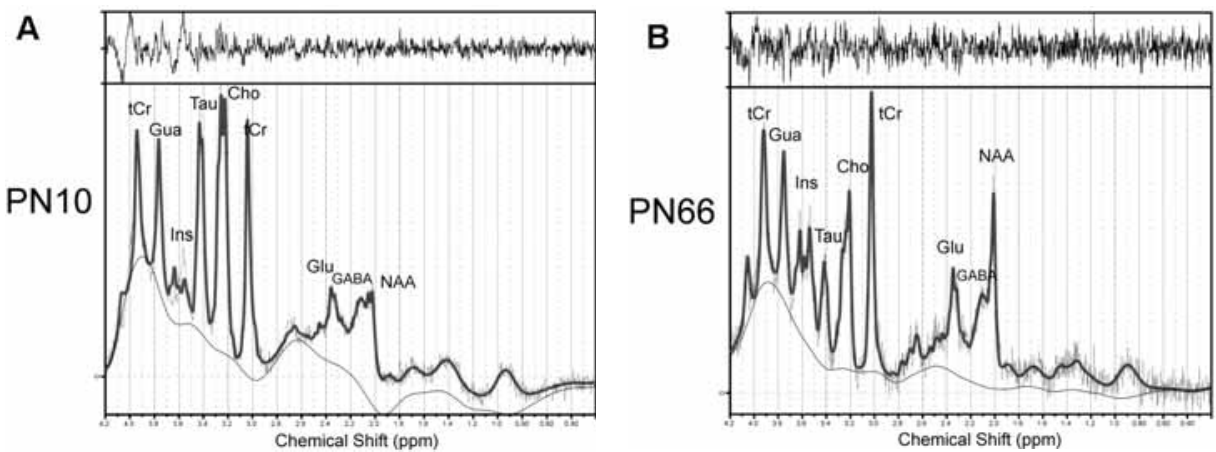

FIG. 3. Analysis output of localized ${ }^{1} \mathrm{H}-\mathrm{MRS}$ spectra obtained at postnatal days (PN) 10 (A) and $66(B)$ in the hippocampus of a hyperthermia treated rat $(\mathrm{HT}+)$. The in vivo spectrum (thin grey curve) has been estimated with the LCModel output (thick black curve), and the difference of these spectra is plotted at the top. The thick gray curve at the bottom indicates the fitted macromolecule baseline. NAA, N-acetylaspartate; $\mathrm{tCr}$, total creatine; Cho, choline; Ins, myo-inositol; Tau, taurine; Glu, glutamate; GABA, gamma-aminobutyric acid; Gua, Guanideacetate.

\section{Histological analysis}

Quantitative results of the histological analyses are presented in table 2.

Hippocampal volumetry. No significant statistical differences between the two groups were found on the Nissl stained sections (example in fig. 4A).

Cells and fibers. Histological analysis of cell size and density of hippocampal DGCs revealed no significant difference between the NT control and HT+ rats, nor did the relative volume occupied by the cells within the DGL differ (table 2, fig. 4C). Relative volume occupied by the cells in the defined amygdalar region was decreased in $\mathrm{HT}+$ rats $(\mathrm{p}<0.05)$, with cellular volume appearing to be decreased but that did not reach statistical significance (fig. 5AB). Astrocytic or neuronal cell density in the amygdala did also not differ between the groups (fig. 5D). With the Black-Gold staining no differences were found in fiber densities between NT control and HT+ rats for the total hippocampus, the hilus, and the amygdala (fig. 4BDE and 5C). In HT+ animals, a significantly higher percentage of the DGL was occupied by stained fibers $(15 \%)$, compared to NT controls 
$(10 \%)(p<0.05)$. MFA was significantly higher $(p<0.05)$ in the DGL (fig. $4 F-H)$. Thus, both density and directionality of fibers in the DGL were increased after hyperthermia.

\begin{tabular}{|c|c|c|c|}
\hline \multirow{2}{*}{ Region of Interest } & & \multicolumn{2}{|c|}{ PN73 } \\
\hline & & \multirow{2}{*}{$\begin{array}{c}\text { HT+ } \\
\text { Mean (SEM) }\end{array}$} & \multirow{2}{*}{$\begin{array}{c}\text { NT } \\
\text { Mean (SEM) }\end{array}$} \\
\hline Quantity & & & \\
\hline \multicolumn{4}{|l|}{$\begin{array}{l}\text { Hippocampus } \\
\text { Volume }\end{array}$} \\
\hline & Total hippocampus $^{a}$ & $35.7(1.4)$ & $33.0(0.8)$ \\
\hline & Neurons $(D G C s)^{b}$ & $540(28)$ & $523(24)$ \\
\hline & Relative $^{c}$ & $61(4)$ & $58(7)$ \\
\hline \multicolumn{4}{|l|}{ Density $^{d}$} \\
\hline & Neurons (DGCs) & $1.13(0.04)$ & $1.08(0.09)$ \\
\hline \multicolumn{4}{|l|}{ Area fraction ${ }^{e}$} \\
\hline & Total hippocampus & $12.6(3.5)$ & $20.5(3.0)$ \\
\hline & $D G L$ & $15.0(1.8) \dagger$ & $9.6(0.9)$ \\
\hline & Hilus & $22.5(3.1)$ & $19.7(1.8)$ \\
\hline \multicolumn{4}{|l|}{ MFA $^{\dagger}$} \\
\hline & $D G L$ & $26(2) \dagger$ & $21(2)$ \\
\hline \multicolumn{4}{|l|}{$\begin{array}{l}\text { Amygdala } \\
\text { Volume }\end{array}$} \\
\hline & Neurons $^{b}$ & $815(42)$ & $965(70)$ \\
\hline & Relative $^{c}$ & $15(1) \dagger$ & $20(1.5)$ \\
\hline \multicolumn{4}{|l|}{ Density ${ }^{d}$} \\
\hline & Neurons & $0.19(0.01)$ & $0.21(0.01)$ \\
\hline \multirow{2}{*}{\multicolumn{4}{|c|}{ Area fraction ${ }^{\mathrm{e}}$}} \\
\hline & & & \\
\hline & Amygdala & $6.9(2.0)$ & $4.6(1.7)$ \\
\hline
\end{tabular}

TABLE 2. Quantitative histological results in hyperthermia and control rats.

$\mathrm{HT}+$, hyperthermia group; NT, normothermia control group; PN73, postnatal day 73; SEM, standard error of the mean; DGCs, dentate granule cells; DGL, granule cell layer; MFA, microscopic fractional anisotropy.

${ }^{\text {a }}$ volume in $\mathrm{mm}^{3},{ }^{\mathrm{b}}$ volume in $\mathrm{um}^{3},{ }^{\mathrm{c}}$ relative volume in $\%,{ }^{\mathrm{d}}$ density in $10^{6} / \mathrm{mm}^{3},{ }^{\mathrm{e}}$ area fraction (fiber density measure) in \%, ${ }^{\mathrm{f}} \mathrm{MFA}$ in $\%$

† 2-tailed $p<0.05$ 

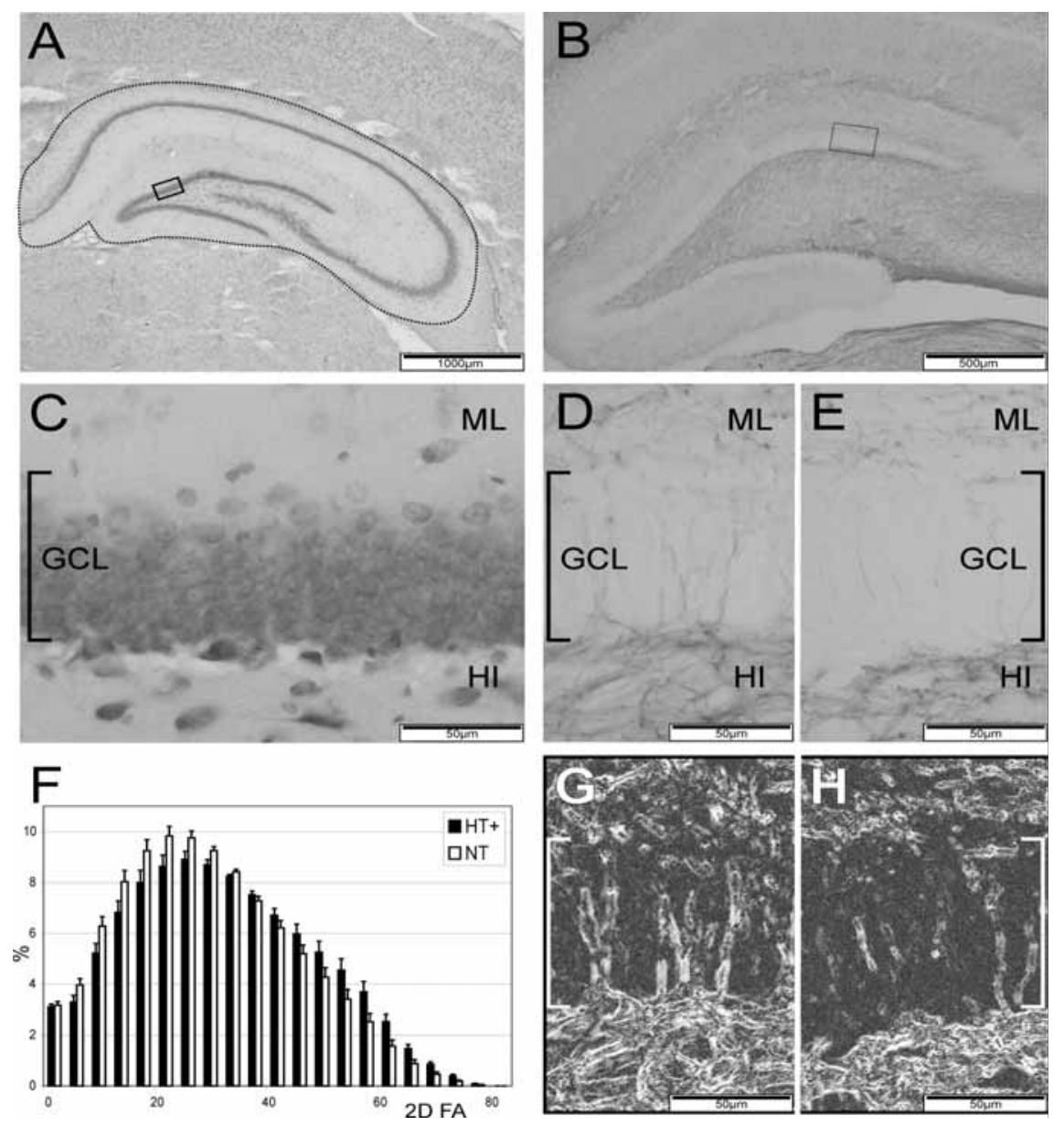

FIG. 4. Histological analyses of the hippocampus, using NissI (A, C) and Black-Gold staining (B, DE, G-H). (A) Delineation of the total hippocampus (dotted line) to calculate the volume. (C) A closeup of the dentate gyrus (detail of the box in $A$ ). Note the molecular layer (ML), the granule cell layer (DGL), and the hilus (HI). With the black-Gold staining, we analyzed fiber density of the total hippocampus (B), and the DGL (D) and $\mathrm{HI}(\mathrm{E})$ (D-E: detail of box in $B$ ). In these pictures (D-E), the granule cells visible in $(C)$ are not stained, leaving a white band (DGL). The Black-Gold stained fibers traverse this DGL well into the ML. Note the densely stained $\mathrm{HI}$ and the increased fiber density of the DGL in picture (D) (example of a HT+ rat) compared to picture (E) (example of a NT rat). (F) Average histogram distribution plots of microscopic fractional anisotropy (MFA) values within the hippocampal DGL. HT+ rats are indicated with black bars, NT rats with white bars. Error bars represent standard error of the mean. Magnified MFA images of the hippocampal DGL for $(G)$ a $\mathrm{HT}+$ and $(\mathrm{H})$ a NT rat. 

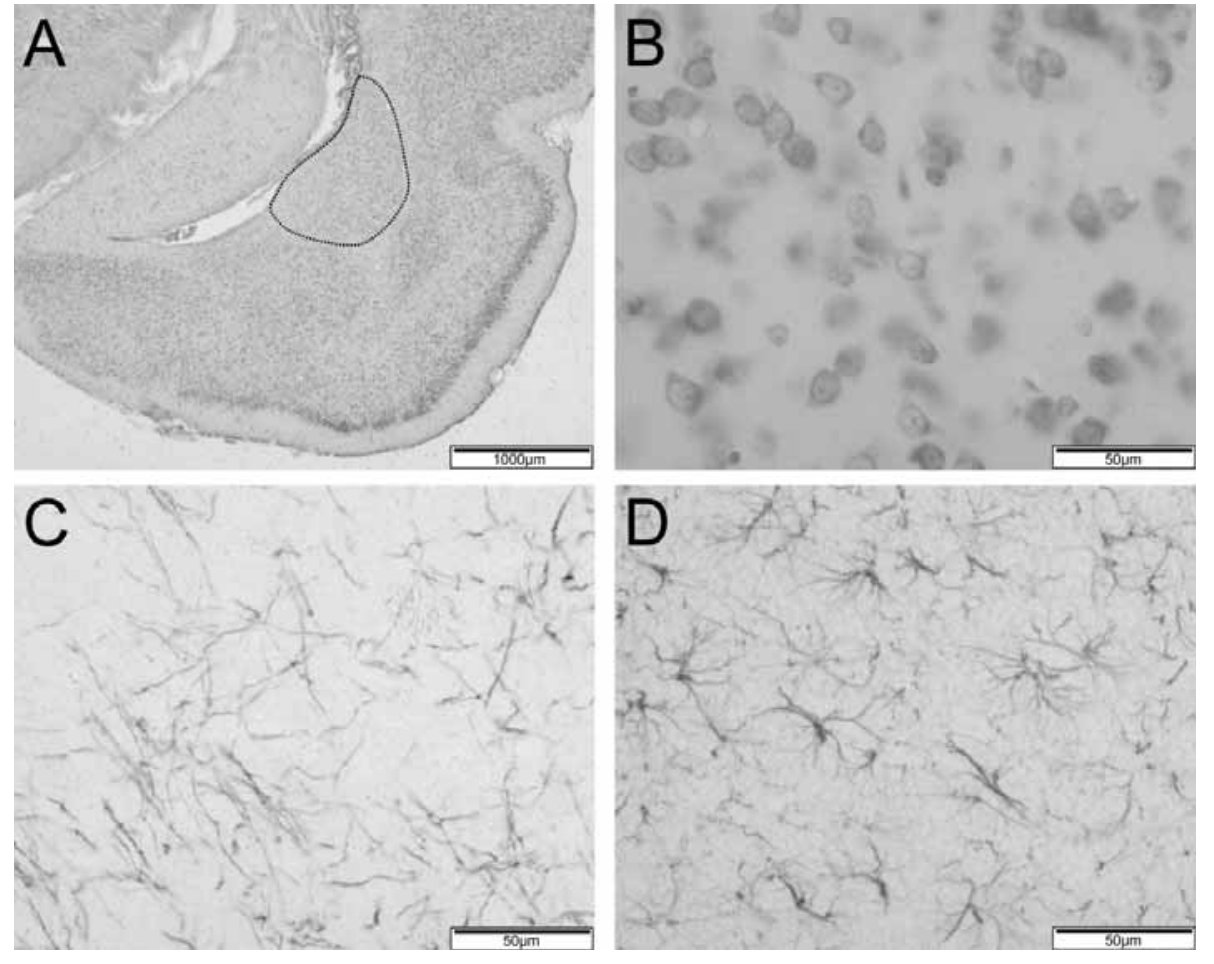

FIG. 5. Histological analyses of the amygdala. (A) Delineation (dotted line) of the lateral amygdala (ventral and dorsal part) using Nissl staining. Detailed view of the neuronal cells (B, Nissl staining), the fibers (C, Black-Gold staining), and the astroglial cells (D, GFAP staining) in the amygdala. 


\section{Discussion}

\section{Current findings}

This study applied quantitative multimodal MRI, comprising hippocampal volumetry and proton spectroscopy, T2 relaxometry, and diffusion weighted imaging, to detect and monitor cerebral FS-induced abnormalities. Experimental FS were shown to induce short-term T2 relaxometric and diffusion changes, particularly in the limbic system. Most importantly, FS provoked long-term diffusion changes in the hippocampus and amygdala, and histological analysis revealed microstructural alterations. Furthermore, FS were shown to cause abnormal changes of the neuronal integrity marker NAA and diffusion anisotropy during hippocampal maturation.

\section{Short-term effects}

The present study revealed elevated quantitative $\mathrm{T} 2$ relaxation times for rats within the hippocampus 24 hours after FS. However, combined analysis of T2 and diffusion further showed abnormalities in the corpus callosum, retrosplenial cortex, piriform cortex, and amygdala but not in the hippocampus. It remains to be elucidated why the hippocampus seems less prone for MRI-detectable alterations. However, these results indicate that, aside from the traditionally most clinically studied structure (hippocampus), other brain regions might yield valuable information on the effects of FS. Acute diffusion abnormalities (i.e. decreased $A D C$ ) in the $H T+$ rats were most prominent in the limbic system (amygdala and piriform cortex, fig. 1EF).

Both T2 elevation and ADC decrease can be interpreted as cytotoxic edema formation (Wall et al., 2000). Elevated T2 relaxation times concur with earlier findings [10] of hyperintense T2-weighted images at 24 hours and 8 days after FS. Quantification of hippocampal volume in the present study revealed no significant difference compared to control rats. Dubé et al also showed that HT- 
induced FS can result in transient cell damage without evolving into cell loss [29]. This might explain why there was a trend towards a decreased NAA value at PN10 and not an obvious decrease, and that acute T2 signal changes resolve over time without persisting neuronal damage. Similarly using amygdala kindling, another relatively mild model of epileptogenesis, others [30] observed increased T2 values with little cell loss or tissue damage, that were not accompanied by differences in hippocampal volume. In contrast, most models of epileptogenesis induce status epilepticus which results in acute tissue degeneration and gives rise to both elevated T2 relaxation time and reduced hippocampal volume [31-33].

\section{Developmental effects}

The observed increase in the neuronal integrity marker NAA [34] from immature to normal adult values was higher in FS rats than controls. NAA normalization might indicate metabolic recovery during maturation [35]. This maturation was abnormal as also indicated by FA reflecting microstructural changes. In line with the current findings, Toth et al [29] showed that acute cell injury did not result in eventual cell loss. Furthermore, it was previously found [13] that experimental FS increase the population of new cells in the hippocampus with $25 \%$, without affecting the cellular density. Therefore, it was suggested that cells are dying but that they are replaced by new cells. This might further explain why NAA was decreased shortly after FS and regained a normal level 8 weeks later. The strong increase of NAA (and decrease of taurine) over time in control rats agrees with earlier work on developmental changes of the rat brain [18] and indicates that development was normal in these rats.

\section{Long-term effects}

The most important findings are the long-term MR changes in the hippocampus (combined analysis of T2, ADC, and FA) and amygdala (decreased ADC). Off all limbic structures that were analyzed, the hippocampus is the one structure 
that does not show acute changes at PN10 when T2, ADC, and FA are considered together. However, it is the only limbic structure that shows later effects at PN66. This suggests a spatial and temporal specific order of events, likely influenced by the plasticity of the developing brain. Therefore, the delayed MRI-observable tissue changes in the hippocampus indicate an ongoing process and could result from seizures [11]. Long-term MR derived hippocampal volumes did not differ between the FS and control group, which was confirmed by histology.

In previous work it was hypothesized that diffusion changes due to seizures could be attributed to several processes including glial hypertrophy, cytoskeletal protein changes, and sprouting of fibers [36]. To interpret the cellular changes underlying the persistent MR results in the hippocampus and amygdala, cell volumes and density were analyzed, combined with microscopic fiber density and anisotropy. Analyses of cell volume and density revealed no changes. In contrast, neuronal cells occupied less of the total amygdalar volume, resulting in an increased volume fraction of the interstitial space. To evaluate the possible contribution of gliosis, known to occur after seizures [37] astrocyte density was assessed in the amygdala. However, no effects were found. Furthermore, fiber density was normal in the amygdala. Interestingly, microscopic fiber density and anisotropy in the dentate gyrus of the hippocampus were increased, possibly indicating mossy fiber sprouting as previously observed [38].

Although histology provided evidence of microstructural changes that are linked to the long-term MR diffusion abnormalities detected, the histological changes (increased volume fraction of the interstitial space) do not explain the observed MRI changes (decreased ADC). However, interpretation of diffusion data remains difficult. Histological changes (e.g. cell swelling/shrinkage, as well as alterations in intercellular space, cellular reorganization, neuronal and fiber density) were determined at a much smaller spatial scale than the voxel size of MRI. The net effect of all changes may reflect either reduced or enhanced water diffusion $[4,39]$. It is therefore unlikely that MR diffusion parameters or changes 
thereof can be explained by a single histological marker. We hypothesize that these changes in ADC, microscopic fiber density, and anisotropy could be attributed to several processes that are involved in the cascade leading from acute to persistent neuronal modifications of the neuronal circuit organization [40], which requires further investigation.

In the epilepsy literature, creatine $(\mathrm{Cr})$ has been proven to be of major interest. It has for example been shown in patients with TLE [41] that the temporal lobe ipsilateral to the seizure focus displays a significant increase in total $\mathrm{Cr}$. A similar finding has also been reported in patients with frontal lobe epilepsy [42]. The high $\mathrm{Cr}$ observed for the HT+ animals in this study may be connected to an increased metabolic rate due to epileptic activity of the tissue, or it may reflect high metabolic activity of the glial cells [43]. However, the actual nature of $\mathrm{Cr}$ changes in epilepsy still remains to be elucidated.

\section{Clinical relevance}

HT-induced FS in rats is proposed as an appropriate-aged model of FS in children [12,44]. Namely, the time point at which FS are induced (i.e. PN9-11) coincides with the period of brain maturation in rats which is most comparable to the maturation stage of the human brain when it is most susceptible for developing FS. However, precise translation remains problematic, partly due to inherent differences between rodents and humans. For instance, several clinical studies have demonstrated hippocampal atrophy on follow-up (2-18 months after FS) $[6-8,45,46]$. Atrophy was not found at the end time-point in the current rat model, which may be at a different stage of epileptogenesis. The relevant analogies, particularly the correspondence of the rat brain development to the FS susceptible period in human infants, were previously summarized [44]. Both the HT-induced FS in rats and FS in humans [5,6,46-48] cause acute transient hippocampal T2 relaxation time elevations, which seem to normalize over time. However, the combined analysis of hippocampal T2, ADC, and FA displayed a chronic effect in the rat hippocampus, a subtle effect that also might be present 
in children, and can be an important early marker of epileptogenesis. Further longitudinal research in which MR findings are directly linked with seizure activity and pathology is required to investigate this potential early marker of epileptogenesis and its underlying mechanism. Long-term longitudinal MR studies, using DTI and spectroscopy, in human children who had FS are lacking up to now and should be encouraged to reveal such possible chronic MR abnormalities during development and their relation with epilepsy. Additionally, the combination of the animal model used and serial multimodal MRI assessments presented may in future be valuable to evaluate anticipatory treatment of FS.

\section{Limitations}

The current study has some limitations that restrict the general validity with respect to its clinical relevance. It is not possible to differentiate whether the observed MR and histological abnormalities are due to hyperthermia itself or FS. Though, the same argument is valid for clinical studies of FS in children for which it is impossible to rule out the effect of the fever itself. To exclude the potential effect of hyperthermia from the effects of FS, an additional control group must be used that consists of littermates that are subjected to the same HT treatment, but seizures are blocked by pretreatment with the barbiturate pentobarbital.

Furthermore, as there was no registration of seizure activity in rats using combined EEG and video monitoring, it is not clear whether the rats with the strongest MR-observable alterations would indeed develop -or had developedepilepsy. The observed MRI alterations in the HT+ animals could be due to seizure activity shortly before MRI examination, or due to long-term epileptogenic processes. However, in either case both causes are linked to the development of epilepsy. 


\section{Conclusion}

Using non-invasive MRI, for the first time it was shown that early-life HTinduced FS give rise to both transient and foremost chronic micro-structural changes to the limbic system (hippocampus and amygdala). The long-term findings support the concept that early-life FS are related to TLE. Whether the detected MRI and histological abnormalities are specific for epileptogenesis or are the consequence of epileptiform activity remains to be elucidated. 


\section{References}

1. Cendes, F., Febrile seizures and mesial temporal sclerosis. Curr. Opin. Neurol., 2004, 17(2), 161-164.

2. Offringa, M., Hazebroek-Kampschreur, A.A., Derksen-Lubsen, G., Prevalence of febrile seizures in Dutch schoolchildren. Paediatr. Perinat. Epidemiol., 1991, 5(2), 181-188.

3. Ojemann, G.A., Temporal lobe epilepsy -current wisdom. Stereotact. Funct. Neurosurg., 2001, 77(1-4), 213-215.

4. Tofts, P., Quantitative MRI of the brain measuring changes caused by disease. 2003, Chichester, West Sussex ; Hoboken, N.J.: John Wiley \& Sons Ltd. 633.

5. Scott, R.C., et al., Abnormalities in hippocampi remote from the seizure focus: a T2 relaxometry study. Brain, 2003, 126(9), 1968-1974.

6. Scott, R.C., et al., Hippocampal abnormalities after prolonged febrile convulsion: a longitudinal MRI study. Brain, 2003, 126(11), 2551-2557.

7. Sokol, D.K., et al., From swelling to sclerosis: acute change in mesial hippocampus after prolonged febrile seizure. Seizure, 2003, 12(4), 237-240.

8. VanLandingham, K.E., et al., Magnetic resonance imaging evidence of hippocampal injury after prolonged focal febrile convulsions. Ann. Neurol., 1998, 43(4), 413-426.

9. Grohn, O.H., Pitkanen, A., Magnetic resonance imaging in animal models of epilepsy Noninvasive detection of structural alterations. Epilepsia, 2007, 48(Suppl. 4), 3-10.

10. Dube, C., et al., Serial MRI after experimental febrile seizures: Altered T2 signal without neuronal death. Ann. Neurol., 2004, 56(5), 709-714.

11. Dube, C., et al., Temporal lobe epilepsy after experimental prolonged febrile seizures: prospective analysis. Brain, 2006, 129(4), 911-922.

12. Baram, T.Z., Gerth, A., Schultz, L., Febrile seizures: an appropriate-aged model suitable for long-term studies. Brain Res. Dev. Brain Res., 1997, 98(2), 265-270.

13. Lemmens, E.M., et al., Gender differences in febrile seizure-induced proliferation and survival in the rat dentate gyrus. Epilepsia, 2005, 46(10), 1603-1612.

14. Dube, C., et al., Prolonged febrile seizures in the immature rat model enhance hippocampal excitability long term. Ann. Neurol., 2000, 47(3), 336-344.

15. Zar, J.H., Biostatistical analysis. 4th ed. 1999, Upper Sadle River, N.J.: Prentice Hall.

16. Barker, P.B., et al., Quantitation of proton NMR spectra of the human brain using tissue water as an internal concentration reference. NMR Biomed., 1993, 6, 89-94.

17. de Graaf, R.A., et al., High magnetic field water and metabolite proton T1 and T2 relaxation in rat brain in vivo. Magn. Reson. Med., 2006, 56(2), 386-394.

18. Tkac, I., et al., Developmental and regional changes in the neurochemical profile of the rat brain determined by in vivo 1H NMR spectroscopy. Magn. Reson. Med., 2003, 50(1), 24-32. 
19. Rorden, C., Brett, M., Stereotaxic display of brain lesions. Behav. Neurol., 2000, 12(4), $191-$ 200.

20. Paxinos, G., Watson, C., The Rat Brain in Stereotaxic Coordinates. 4th ed. 1998, San Diego, CA: Academic Press.

21. Cavassila, S., et al., Cramer-Rao bounds: an evaluation tool for quantitation. NMR Biomed., 2001, 14(4), 278-283.

22. Schmued, L., Slikker, W., Jr., Black-gold: a simple, high-resolution histochemical label for normal and pathological myelin in brain tissue sections. Brain Res., 1999, 837(1-2), 289-297.

23. Thom, M., et al., Cytoarchitectural abnormalities in hippocampal sclerosis. J. Neuropathol. Exp. Neurol., 2002, 61, 510-519.

24. Gundersen, H.J., et al., The new stereological tools: disector, fractionator, nucleator and point sampled intercepts and their use in pathological research and diagnosis. Apmis, 1988, 96(10), 857-881.

25. Marple, S.L., Digital spectral analysis: with applications. 1987, Englewood Cliffs, N. J.: Prentice Hall.

26. Läuter, J., Exact $\mathrm{T}$ and $\mathrm{F}$ tests for analyzing studies with multiple endpoints. Biometrics, 1996, 52, 964-970.

27. O'Brien, P.C., Procedures for comparing samples with multiple endpoints. Biometrics, 1984, 40(4), 1079-1087.

28. Hochberg, Y., A sharper Bonferroni procedure for multiple tests of significance. Biometrika, 1988, 75, 800-802.

29. Toth, Z., et al., Seizure-induced neuronal injury: vulnerability to febrile seizures in an immature rat model. J. Neurosci., 1998, 18(11), 4285-4294.

30. Jupp, B., et al., Hippocampal T2 signal change during amygdala kindling epileptogenesis. Epilepsia, 2006, 47(1), 41-46.

31. Nairismagi, J., et al., Progression of brain damage after status epilepticus and its association with epileptogenesis: a quantitative MRI study in a rat model of temporal lobe epilepsy. Epilepsia, 2004, 45(9), 1024-1034.

32. Nairismagi, J., et al., Status epilepticus in 12-day-old rats leads to temporal lobe neurodegeneration and volume reduction: a histologic and MRI study. Epilepsia, 2006, 47(3), 479-488.

33. Nairismagi, J., et al., Manganese-enhanced magnetic resonance imaging of mossy fiber plasticity in vivo. Neuroimage, 2006, 30(1), 130-135.

34. Maton, B.M., Kuzniecky, R.I., Proton MRS: N-acetyl aspartate, creatine, and choline. Adv. Neurol., 2000, 83, 253-259.

35. Hugg, J.W., et al., Normalization of contralateral metabolic function following temporal lobectomy demonstrated by $1 \mathrm{H}$ magnetic resonance spectroscopic imaging. Ann. Neurol., 1996, 40(2), 236-239. 
36. Ben-Ari, Y., Cell death and synaptic reorganizations produced by seizures. Epilepsia, 2001, 42(Suppl. 3), 5-7.

37. Ravizza, T., et al., Inflammatory response and glia activation in developing rat hippocampus after status epilepticus. Epilepsia, 2005, 46(Suppl. 5), 113-117.

38. Bender, R.A., et al., Mossy fiber plasticity and enhanced hippocampal excitability, without hippocampal cell loss or altered neurogenesis, in an animal model of prolonged febrile seizures. Hippocampus, 2003, 13(3), 399-412.

39. Liu, K.F., et al., Regional variations in the apparent diffusion coefficient and the intracellular distribution of water in rat brain during acute focal ischemia. Stroke, 2001, 32(8), 1897-1905.

40. Wall, C.J., Kendall, E.J., Obenaus, A., Rapid alterations in diffusion-weighted images with anatomic correlates in a rodent model of status epilepticus. Am. J. Neuroradiol., 2000, 21(10), 1841-1852.

41. Connelly, A., et al., Magnetic resonance spectroscopy in temporal lobe epilepsy. Neurology, 1994, 44, 1411-1417.

42. Lundbom, N., et al., Proton spectroscopic imaging shows abnormalities in glial and neuronal cell pools in frontal lobe epilepsy. Epilepsia, 2001, 42, 1507-1514.

43. Urenjak, J., et al., Proton nuclear magnetic resonance spectroscopy unambiguously identifies different neural cell types. J. Neurosci., 1993, 13, 981-989.

44. Jensen, F.E., Baram, T.Z., Developmental seizures induced by common early-life insults: short- and long-term effects on seizure susceptibility. Ment. Retard. Dev. Disabil. Res. Rev., 2000, 6(4), 253-257.

45. Farina, L., et al., Acute diffusion abnormalities in the hippocampus of children with new-onset seizures: the development of mesial temporal sclerosis. Neuroradiology, 2004, 46(4), 251257.

46. Scott, R.C., et al., Prolonged febrile seizures are associated with hippocampal vasogenic edema and developmental changes. Epilepsia, 2006, 47(9), 1493-1498.

47. Scott, R.C., et al., Quantitative magnetic resonance characterization of mesial temporal sclerosis in childhood. Neurology, 2001, 56(12), 1659-1665.

48. Scott, R.C., et al., Magnetic resonance imaging findings within 5 days of status epilepticus in childhood. Brain, 2002, 125(9), 1951-1959. 


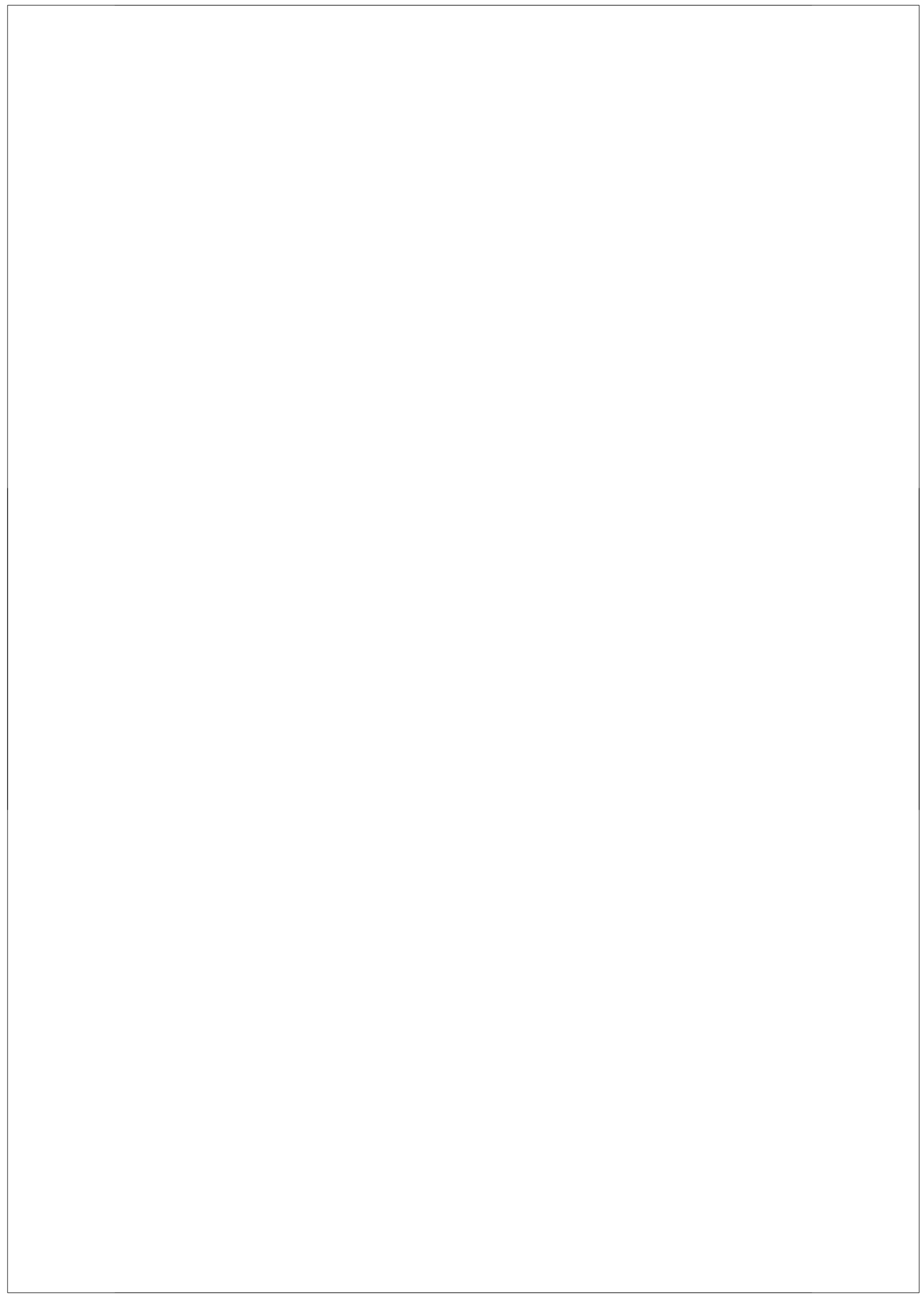



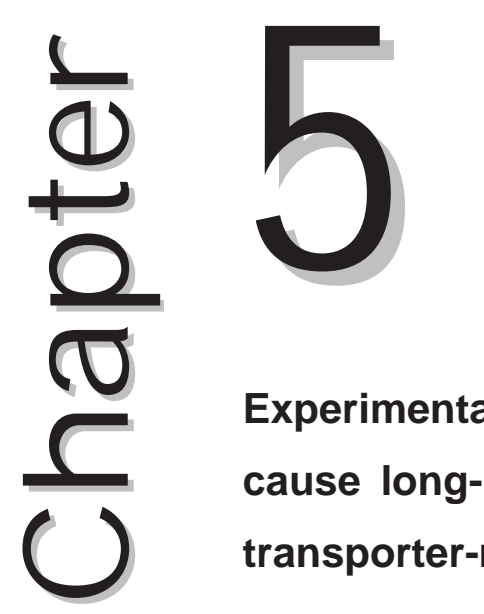

Experimental febrile seizures do not cause long-lasting changes in glutamate transporter-mediated neurophysiology

Evi M.P. Lemmens, Kim Rijkers, Vivianne van Kranen-Mastenbroek, Olaf E.M.G. Schijns, Emile A.M. Beuls, Govert Hoogland

\section{ABSTRACT}

Febrile seizures (FS), i.e. seizures that are accompanied by high fever and which occur only in young children, have been causally linked to the development of temporal lobe epilepsy. Animal models previously showed hyperexcitability and spontaneous seizures after early-life FS, but the mechanisms are unknown. Deficient glutamate uptake has been proven to be involved in epilepsy and seizures. In the present study we measured glutamate transporter (EAAT) expression and function after experimental FS.

FS were induced by hyperthermia ( $\mathrm{HT} ; 30 \mathrm{~min}, 41^{\circ} \mathrm{C}$ core temperature) in 10-day old rats and littermates were used as normothermia controls (NT; $30 \mathrm{~min}, 35^{\circ} \mathrm{C}$ ). Eight weeks after FS, hippocampal EAAT1, 2, and 3 expression was evaluated by quantitative immunohistochemistry. Transporter function was measured in a separate group of rats by EEG recordings following the administration of the glutamate transporter blocker DLthreo- $\beta$-Benzyloxyaspartic acid (TBOA)

Expression of EAAT1, 2, and 3 was not changed after HT compared to NT treatment. No behavioral or electroencephalographic seizures were detected after blocking glutamate transport.

Early-life FS do not cause changes in hippocampal glutamate transporter expression or function in adult rats. 


\section{Introduction}

As glutamate and GABA are the major excitatory and inhibitory neurotransmitters in the brain, it is not surprising that they are involved in many aspects of normal and pathological brain functioning. Excessive amounts of glutamate are harmful and neurotoxic [1], thus it is very important that the level of glutamate available to bind to its receptors is precisely regulated. When glutamate is released, there is only one efficient way to remove it from the extracellular space, which is through uptake by glutamate transporters. Until today, five isoforms of the glutamate transporter, also referred to as "excitatory amino acid transporter" (EAAT), have been identified. These were named EAAT1/GLAST and EAAT2/GLT-1, both present on glial cells, EAAT3/EAAC1 and EAAT4, both expressed by neurons, and EAAT5 which was found to be primarily expressed in the retina (reviewed in [2]).

Hyperexcitability in epilepsy, as a result of an imbalance between excitatory and inhibitory neurotransmission, could be due to ineffective removal of glutamate from the synaptic cleft during synaptic transmission and dysregulation of glutamate metabolism. Human studies of temporal lobe epilepsy (TLE), one of the most common forms of epilepsy in adults, have provided evidence of alterations of glutamate and GABA concentrations accompanying seizure activity. For example, in vivo microdialysis of the hippocampus of TLE patients revealed an already elevated extracellular glutamate concentration before seizures, which increased even further preceding and during seizures [3]. Furthermore, Petroff et al. [4] showed slowing of the glutamate-glutamine cycling in epilepsy patients, resulting in an increased level of glutamate. Human studies performed using resected tissue from TLE patients with hippocampal sclerosis (HS), have reported alterations in the expression levels of the different glutamate transporters. Mathern et al. [5] found a decrease in EAAT2 immunoreactivity (IR) and an increase in EAAT1 and EAAT3 IR in sclerotic tissue compared to non-sclerotic and autopsy control tissue. Crino et al. [6] showed an upregulation of EAAT3 in epileptic tissue without a change in EAAT2 expression while Proper et al. [7] found decreased EAAT1 and EAAT2, as well 
as a decreased number of EAAT3 expressing cells but they also demonstrated that the amount of EAAT3 per cell increased. Furthermore, Hoogland et al. [8] have shown that synaptosomal glutamate uptake is decreased in TLE tissue. Another study by Tessler et al. however, failed to show alterations in EAAT levels in epilepsy patients [9].

Studies aimed at decreasing the expression of glutamate transporters in animal models of epilepsy have strengthened the idea of a causal relationship between deficient glutamate uptake and seizure activity [10]. In this respect, reducing GLAST and GLT-1 expression by silencing caused extensive neurotoxicity, while a reduction in EAAC1 led to seizures [11,12]. GLAST knockout mice have been shown to display increased seizure susceptibility but no spontaneous seizures [13]. In another study, EAAC1 deficient mice did not develop neurodegeneration nor epilepsy [14]. However, GLT-1 knockout animals show spontaneous seizures that were often lethal [15]. Pharmacologically blocking glutamate transport has led to similar conclusions about the role of EAATs in seizure activity. In vitro (brain slices), as well as in vivo (intraventricular injection) experiments have demonstrated that treatment with DL-threo- $\beta$ Benzyloxyaspartic acid (TBOA) for example, can induce synchronous oscillations and seizures [16-18].

The development of HS-associated TLE in adults has often been linked to the occurrence of early-life febrile seizures (FS) [19]. Using an animal model of hyperthermia (HT)-induced FS, it was previously shown that FS animals develop hyperexcitability and eventually spontaneous seizures later in life $[20,21]$. Also, these animals demonstrated an increased proliferation of dentate granule cells (DGCs) but a decrease in the number of newborn cells that developed into EAAC1 expressing cells $[22,23]$. The aim of the present study was to check the functional implications of the decrease in EAAC1 found previously, and thus the role of glutamate transport in hyperexcitability and the development of spontaneous seizure activity induced by early-life FS. Therefore, we first analyzed overall levels of GLAST, GLT-1, and EAATC1 in the hippocampus of FS animals, and secondly, measured changes in brain activity induced by pharmacologically blocking of glutamate transport by TBOA. 


\section{Materials and Methods}

\section{Animal model}

Male Sprague Dawley rats (Harlan, The Netherlands) were born and housed under standard conditions. Experiments were approved by the local Animal Experiments Committee of Maastricht University and conformed to governmental policies.

HT was induced as described previously [22,24]. In brief, 10-day-old rat pups were placed in a cylinder and their core temperature was raised with heated air to $41-42.5^{\circ} \mathrm{C}$, at which it was maintained for 30 minutes. Core temperatures were measured before and every $2.5 \mathrm{~min}$ during the HT treatment. The behavioral seizures were stereotyped and previously shown to correlate with hippocampal EEG discharges [20]. A subset of rats showed FS behavior during HT (HT+ rats; $n=18)$ while other rats did not (HT- rats; $n=10)$. Littermates were used as normothermia (NT; $n=17$ ) controls, meaning that they were exposed to the same conditions, except that the physiological core temperature of the rats was maintained. At PN21, all pups were weaned and randomly housed, two to three rats per cage.

\section{Electro graphical recording}

\section{Electrode implantation}

To measure brain activity, 7 weeks after HT or NT treatment an electrode headset was implanted in a subset of NT control $(n=10)$ and HT+ $(n=11)$ rats. The headset consisted of 4 stainless steel injection needles, attached to a printed circuit board and to that a connector (custom-made by the department of Instrument Development Engineering and Evaluation (IDEE), Maastricht University). Prior to surgery, rats were injected subcutaneously with buprenorfine $(0.1 \mathrm{mg} / \mathrm{kg})$ for pain relief. Then, rats were anesthetized with 
isoflurane and placed in a stereotactic apparatus (Stoelting, Wood Dale, USA). Hypothermia was prevented by using a heated water pad, and dehydration of the eyes by application of sterile Vaseline. Lidocaine $(10 \mathrm{mg})$ was injected subcutaneously before a midline incision was made; then the skin was retracted and the periost was removed. To improve adherence of the electrode headset to the skull, an etching gel was used containing phosphoric acid (ID blue gel TM; Innovations Dentaires, Dongen, the Netherlands). Four burr holes were made in the skull for correct positioning of the electrodes. Recording electrodes were placed in the left dorsal hippocampus $(\varnothing 0.3 \mathrm{~mm}$; Bregma coordinates: AP= $-3.7 \mathrm{~mm}, \mathrm{~L}=2.7 \mathrm{~mm}, \mathrm{~V}=-3.7 \mathrm{~mm}$ ), and in the right frontal cortex $(\varnothing 0.5 \mathrm{~mm}$; Bregma coordinates: $\mathrm{AP}=-3.7 \mathrm{~mm}, \mathrm{~L}=4.8 \mathrm{~mm}, \mathrm{~V}=-2.7 \mathrm{~mm})$. The third electrode was situated in the left visual cortex and used as ground $(\varnothing 0.5 \mathrm{~mm}$; Bregma coordinates: $A P=-7.7 \mathrm{~mm}, \mathrm{~L}=4.8 \mathrm{~mm}, \mathrm{~V}=-4.5 \mathrm{~mm}$ ), and the fourth in the right occipital cortex (ø $0.5 \mathrm{~mm}$; used as reference; Bregma coordinates: $\mathrm{AP}=-7.7 \mathrm{~mm}, \mathrm{~L}=4.8 \mathrm{~mm}, \mathrm{~V}=-4.5 \mathrm{~mm}$ ) [25]. Three stainless steel screws were used to anchor the headset with dental cement to the skull.

\section{EEG monitoring}

Starting one week after electrode implantation, EEG was recorded using a Vangard system (Cleveland Clinic Foundation). After recording a baseline EEG for $15 \mathrm{~min}$, the animals were placed in a restrainer and injected intravenously with a solution of TBOA (100 $\mu \mathrm{l} ; 8 \mathrm{mM})$ and mannitol $(1.5 \mathrm{~g} / \mathrm{kg}$ body weight dissolved in $0.9 \%$ saline), immediately followed by an additional 30 min EEG recording. Behavior was observed by two investigators blind to the treatment history of the animals, especially focused on the occurrence of distinct behavioral alterations such as facial automatisms (i.e. chewing, salivation), head nodding, forelimb clonus, wild running or freezing, and wet dog shakes. One week later, the EEG was recorded again for 60 minutes. Recordings were made with a sample frequency of $200 \mathrm{~Hz}$, frequency band of $0.5-70 \mathrm{~Hz}$ and with a $50 \mathrm{~Hz}$ Notch filter. EEG data was analyzed visually. 


\section{Histology}

Rats were sacrificed by transcardial perfusion with $4 \%$ paraformaldehyde under pentobarbital anesthesia, and the brains were removed, post fixated for 48 hours, cryoprotected in $30 \%$ sucrose. Coronal serial sections $(10 \mu \mathrm{m}$ thickness; $300 \mu \mathrm{m}$ inter-section distance) throughout the hippocampus were cut using a cryostat and stored at $-20^{\circ} \mathrm{C}$ until further use.

One series of sections was stained using hematoxylin-eosin (HE) to confirm the position of the hippocampal electrode. Three other series of sections were immunohistochemically stained for the glial glutamate transporters GLAST and GLT1, and the neuronal glutamate transporter EAAC1. Blocking of endogenous peroxidase and nonspecific binding was achieved by incubation with Trisbuffered saline containing $0.3 \%$ Triton $\mathrm{X}-100$ (TBS-T) containing $3 \% \mathrm{H}_{2} \mathrm{O}_{2}$ and $3 \%$ normal donkey serum (NDS), respectively. The following antibodies and conditions were used: (1) primary antibodies: monoclonal mouse anti-GLAST (1:100) and anti-GLT1 (1:200; Novocastra Laboratories Ltd, Newcastle, UK) incubated with for 48 hours at $4^{\circ} \mathrm{C}$, and polyclonal rabbit anti-EAAC1 (1:500; Alpha Diagnostics Intl. Inc., San Antonio, TX, USA) incubated with for 24 hours at $4{ }^{\circ} \mathrm{C}$; (2) secondary antibodies: biotinylated donkey anti-mouse antibody and biotinylated donkey anti-rabbit (1:400; Jackson Immunoresearch Laboratories, West Grove, PA, USA) incubated with for $60 \mathrm{~min}$ at room temperature. All primary antibodies were diluted in TBS-T containing 1.5\% (NDS). Staining was visualized using the Vectastain method (Vector Laboratories, Burlingame, CA, USA) and diaminobenzidine.

Stainings were analyzed using an Olympus AX70 microscope with bright field illumination. Pictures were made with an F-view cooled CCD camera and analyzed using CellP software (Olympus, the Netherlands). In total, 5 hippocampal sections were used per staining, located between Bregma $-1.8 \mathrm{~mm}$ and $-4.8 \mathrm{~mm}$, with an inter-section distance of $600 \mu \mathrm{m}$. In each section, 2 regions of interest $(\mathrm{ROI})$ were investigated, i.e. the dentate gyrus (DG), with the upper and lower blade delineated separately where visible, and the CA3 
area. To assess staining intensity, an upper and lower threshold value was determined for each staining separately in order to distinguish background from specific staining, and kept constant for all measurements of each staining. The lower threshold was determined using a negative control, i.e., a section in which the primary antibody was omitted; the upper threshold was determined from the section with the darkest staining pattern. For each ROI, the following parameters were analyzed using CellP software: (i) area fraction (the percentage of pixels with a grey value within the threshold limits per total number of pixels), and (ii) the mean gray value of the pixels within the threshold. Gray values ranged from 0 to 255 with ' 0 ' being black and ' 255 ' white, so a higher gray value equals less staining.

\section{Statistical analysis}

Statistical significance $(p<0.05)$ between the histological parameters analyzed was determined using analysis of variance (ANOVA) and Bonferroni's multiple comparison post hoc test (SPSS for Windows; Chicago, IL, USA). Data are presented as mean + SEM. 


\section{Results}

\section{Histological verification of hippocampal electrode position}

Evaluation of electrode position using a HE staining verified that in all animals the hippocampal electrode was implanted in the hippocampus (fig. 1). The largest visible electrode trajectories most often included the electrode tip and were situated between Bregma -3.3 and $-3.8 \mathrm{~mm}$, with one exception in which the electrode tip was situated at Bregma $-2.56 \mathrm{~mm}$ (table 1).

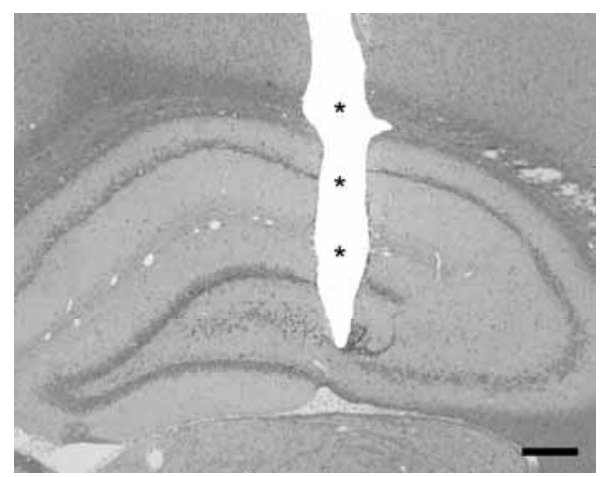

FIG. 1. Hematoxylin-eosin staining of the hippocampus to illustrate the trajectory $\left(^{*}\right)$ of the hippocampal electrode. Note the position of the electrode tip at the border of CA3/4. Scale bar = $400 \mu \mathrm{m}$.

\section{Glutamate transporter expression}

The immunohistochemical staining pattern of GLT-1 showed a ubiquitous expression of this glial glutamate transporter throughout the hippocampus. Cell bodies were immunodeficient and staining was present between the pyramidal cells of the CA3 area (fig. 2A) and the granule cells of the DG (fig. 2B). Some astrocytes were clearly visible (fig. $2 \mathrm{~B}$, boxed detail). GLAST immunoreactivity showed a similar staining pattern (data not shown). On the contrary, EAAC1 staining was present in neuronal cells (fig. 2C, D). 


\begin{tabular}{l||l|l|l} 
animal & treatment & Bregma $(\mathbf{m m})$ & position \\
\hline 1 & NT & -3.3 & CA3 \\
2 & HT+ & -3.6 & CA4 \\
3 & NT & -3.6 & CA1 \\
4 & HT+ & -3.3 & CA3 \\
5 & HT+ & -3.6 & CA1 \\
6 & NT & -3.6 & CA1/2 - CA4 \\
7 & HT+ & -3.6 & CA4 \\
8 & HT+ & -3.8 & DGL upper blade \\
9 & HT+ & -3.3 & DGL upper blade \\
10 & NT & -2.56 & CA3/4 \\
11 & NT & -3.8 & CA1 \\
12 & HT+ & -3.6 & DGL upper blade \\
13 & NT & -3.8 & CA1 \\
14 & NT & -3.6 & CA4 \\
15 & HT+ & -3.6 & CA1 \\
16 & HT+ & -3.8 & DGL lower blade \\
17 & NT & -3.6 & CA1 \\
18 & HT+ & -3.3 & CA1 \\
19 & NT & -3.6 & CA3 \\
20 & NT & -3.3 & CA3 \\
21 & HT+ & -3.3 & CA1 \\
\hline
\end{tabular}

TABLE 1. Position of the hippocampal electrode tip.

NT, normothermia treatment; HT+, hyperthermia-induced seizures; CA, Cornu Ammonis; DGL, granule cell layer of the dentate gyrus. 


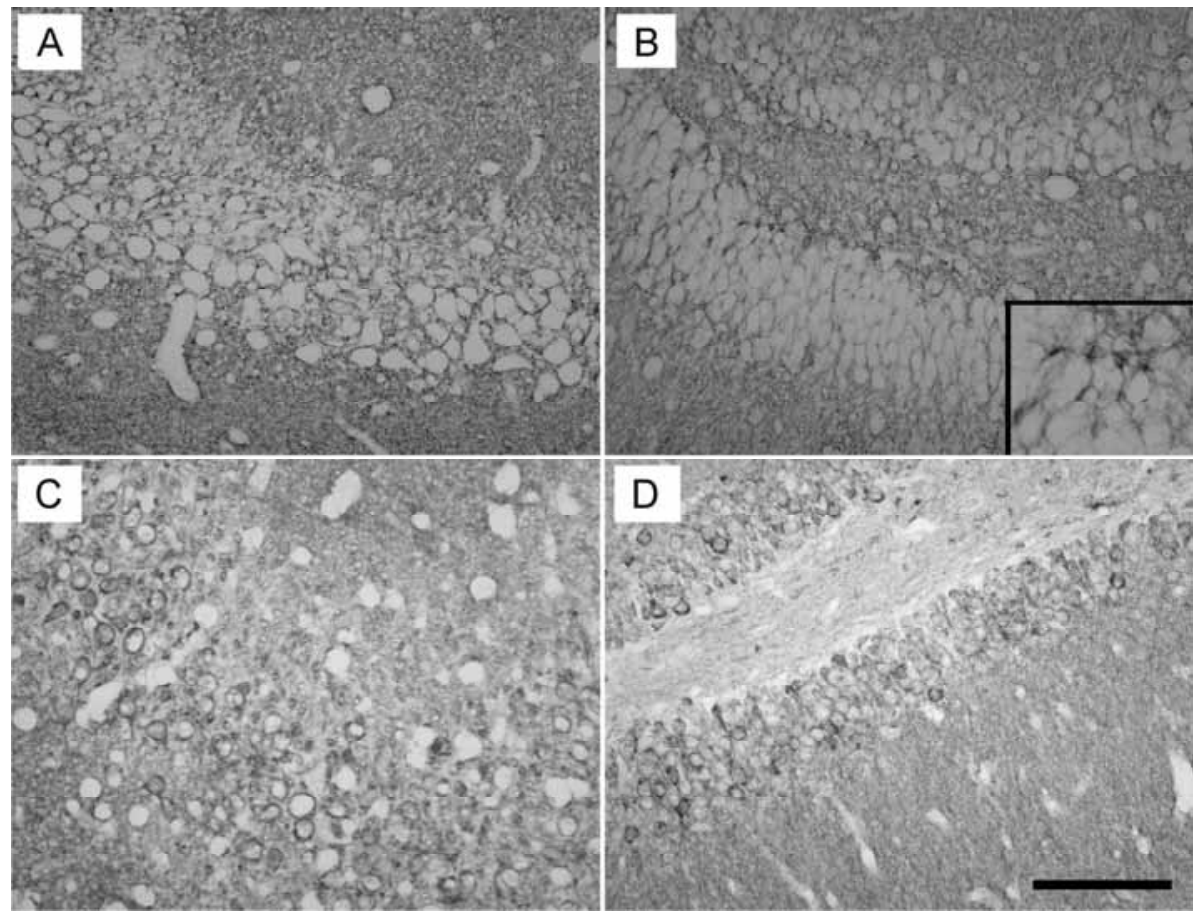

FIG. 2. Photomicrographs of GLT-1 (A,B) and EAAC1 (C,D) immunohistochemical staining of the $C A 3$ area $(A, C)$ and dentate gyrus $(B, D)$ of the hippocampus. Note the astrocyte-like cells situated in the granule cell layer $(B$, boxed detail). Scale bar $=100 \mu \mathrm{m}$.

Quantification of area fraction (a measure of the number of immunoreactive cells; fig. 3) and mean gray value (a measure of the total expression; fig. 4) failed to demonstrate an effect of HT treatment for each of the glutamate transporter stainings, either when comparing NT controls with HT- animals or with $\mathrm{HT}+$ animals. When comparing the EAAT immunoreactivity of TBOAtreated animals with that of non-TBOA-treated animals, the HT+ animals showed a significant $(p<0.01)$ decreased expression of GLAST in the DG and CA3 area, and EAAT3 in the CA3, and a trend towards a decreased expression of GLT-1 for both the DG and CA3, and EAAT3 in the DG. For the NT controls, there was only a trend towards a decreased expression for GLAST, GLT-1 and EAAT3, both in the DG and CA3 region. 

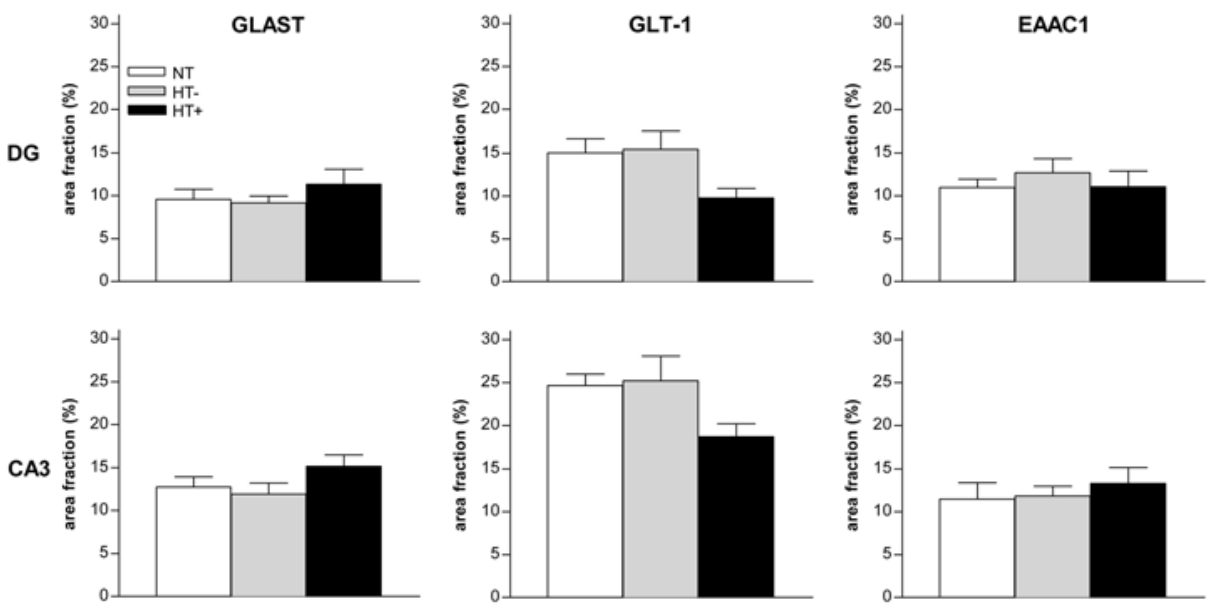

FIG. 3. Quantification of GLAST, GLT-1, and EAAC1 staining of normothermia controls (NT, $n=7$ ), hyperthermia animals without seizures (HT-, $n=10)$, and hyperthermia animals that did experience seizures $(\mathrm{HT}+, \mathrm{n}=7)$. Staining intensity was expressed as the area fraction (i.e. number of 'positive' pixels, as determined by setting threshold values, as percentage (\%) of total number of pixels within the region of interest [dentate gyrus (DG) or CA3]). Neither staining showed a significant treatment effect.
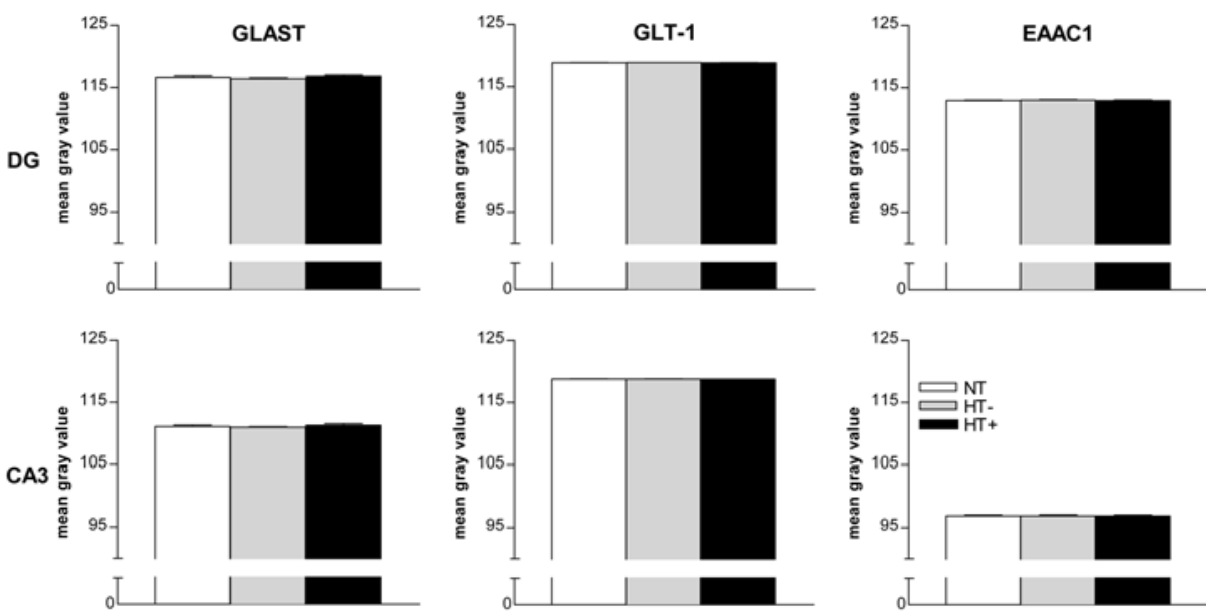

FIG. 4. Analysis of mean gray value for GLAST, GLT-1, and EAAC1 staining within the dentate gyrus (DG) and CA3 area of the hippocampus showed no significant differences between normothermia $(N T ; n=7)$ control animals, hyperthermia animals without seizures $(H T-; n=10)$, and hyperthermia animals that did experience seizures $(\mathrm{HT}+; \mathrm{n}=7)$. 


\section{Visual inspection of the EEG measurements and behavioral observation}

Eight weeks after NT or HT treatment, cortical and hippocampal EEG recordings, as well as behavioral observations were made before and after administering the EAAT antagonist TBOA.

Behavioral observation of the animals after TBOA treatment did not comply with the hypothesis that blocking of glutamate transport induces seizure activity. This result was confirmed by visual inspection of the EEG recordings in which parameters of typical seizure activity (i.e. rhythmic spiking, amplitude increase over time, increased frequency) were absent. Animals showed a short episode of exploratory behavior (few minutes) but were mostly calm and sleepy thereafter. Movement artifacts were only rarely seen.

Figure 5 shows EEG recordings of a NT control animal and two HT+ animals. After TBOA administration, the NT animal showed slowing of the hippocampal and cortical signal, while there were no changes noted for the two HT+ animals. Since the baseline EEG recordings were already different between animals, this made interpretation of the data even more complicated. For example, the hippocampal recording of $\mathrm{HT}+$ animal $n^{\circ} 2$ had a much higher amplitude compared to the recordings of the NT animal and $H T+$ animal $n^{\circ} 1$. Surprisingly, one week after TBOA treatment, the EEG signal was changed in some animals (NT and $H T+n^{\circ} 1$ ), even those that did not show a change immediately after TBOA treatment $\left(H T+n^{\circ} 1\right)$. However, there were still no clear signs of seizure activity (electrographical or behavioral) present.

Some rats showed freezing behavior, chewing, facial clonus, and/or wet dog shakes in the 30 minute of recording immediately after TBOA, but these behaviors occurred without a clear and consistent EEG correlate. For example, freezing was accompanied by an increase in amplitude and/or decreased frequency of the cortical signal in some rats (fig. $6, \mathrm{HT}+\mathrm{n}^{\circ} 4$ ) but not in all rats (fig. $6, \mathrm{HT}+\mathrm{n}^{\circ} 3$ ) when compared to the signal during exploration. 
Overall, behavior and EEG were highly variable among the animals but TBOA did have an effect on brain activity and behavior in some but not in all animals.

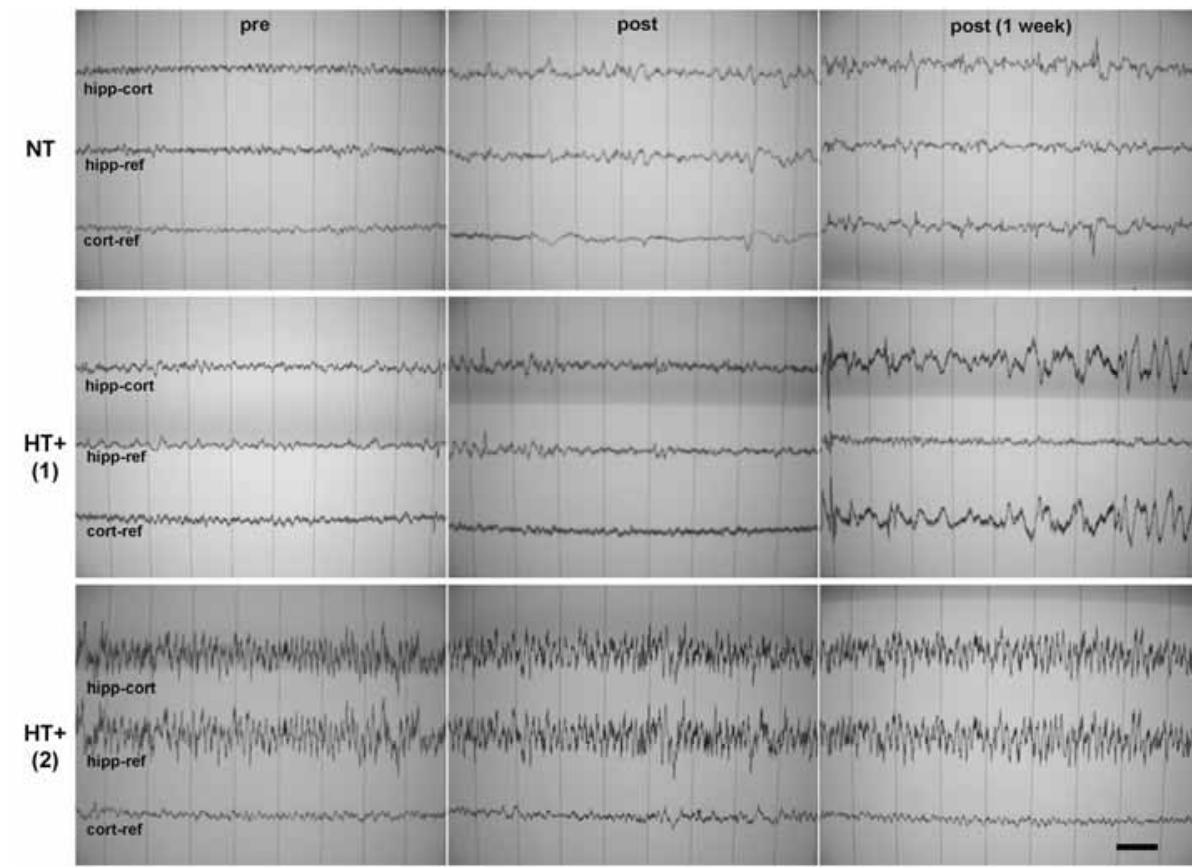

FIG. 5. Panel displaying electroencephalographic (EEG) recordings of a normothermia (NT) control rat, and two rats with hyperthermia-induced seizures $(\mathrm{HT}+)$. One bipolar montage (hipp-cort = hippocampal-cortical electrode), and two reference montages (hipp-ref $=$ hippocampal-reference electrode; cort-ref = cortical-reference electrode) were made. Baseline EEG (pre) recordings were highly variable among the animals; e.g. the hippocampal signal of $\mathrm{HT}+$ rat $\mathrm{n}^{\circ} 2$ had a much higher amplitude than that of the other 2 rats. TBOA injection (post) induced slowing on both the hippocampal and cortical signal of the NT rat while the EEG of the HT+ rats was not changed. One week after TBOA treatment (post, 1 week) the EEG signal was changed in some animals, irrespective of whether the signal was changed immediately after TBOA as for the NT rat, or not changed as for the HT+ rats. 


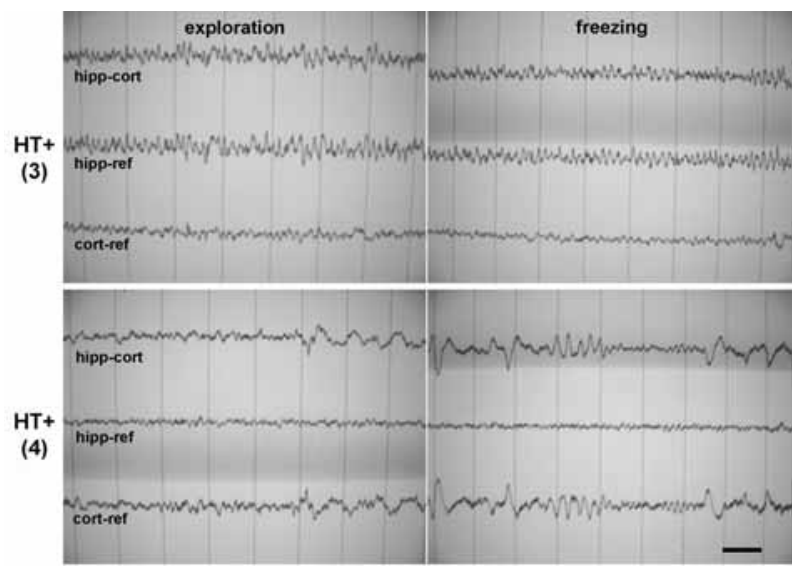

FIG. 6. Examples of electroencephalographic (EEG) recordings during exploration and freezing of two rats with hyperthermia-induced seizures $(\mathrm{HT}+$ ). One bipolar montage (hipp-cort $=$ hippocampalcortical electrode), and two reference montages (hipp-ref = hippocampal-reference electrode; cortref $=$ cortical-reference electrode) were made. Note that the EEG signals of the two HT+ rats was not similar during exploration and that freezing behavior in $\mathrm{HT}+$ rat $\mathrm{n}^{\circ} 3$ was accompanied by a minimal decrease in amplitude of all three signals, while $\mathrm{HT}+$ rat $\mathrm{n}^{\circ} 4$ showed slowing of the cortical signals during freezing. 


\section{Discussion}

There is evidence of a causal link between FS in childhood, and the development of TLE later in life. Hyperexcitability related to FS-induced epileptogenesis, may be due to inefficient glutamate uptake. The results of the present study demonstrated that hyperthermia-induced FS in 10-day old rats (1) do not change the long-term expression of the glial and neuronal glutamate transporters GLAST, GLT-1, and EAAC1; and (2) do not alter the susceptibility to TBOA-evoked seizures. Taken together, these results argue against a causal association between glutamate uptake and FS-induced hyperexcitability.

\section{Early-life experimental FS do not alter hippocampal expression of glutamate transporters in adulthood}

Studies using animal models of seizures and epilepsy have well established a link between an increase in the level of extracellular glutamate and seizure activity (review by [26]). It remains to be determined whether this rise in glutamate is a consequence of increased glutamate release, inefficient glutamate metabolism, or decreased uptake. Evidence from the literature suggests that changes in the expression of glutamate transporters are involved. In this respect, it was shown by Northern and Western blotting, and immunohistochemistry, that in adult rats the expression of hippocampal EAAC1 mRNA and protein is increased after amygdala kindling [27] and kainic acid injection [28], tetanic hippocampal stimulation [29], and pilocarpine-induced status epilepticus (SE) [6]. This effect was not only observed when seizures were induced in adult rats, but also after early-life seizures in 10-day old rat pups when pilocarpine was used to induce SE and transporter expression in the DG was measured in adulthood [30]. Often the increased EAAC1 expression was accompanied by neurodegeneration, and thought to be a compensatory mechanism for the loss of EAAC1 expressing neurons. Results of the expression of GLAST and GLT-1 were less consistent; mostly, no change in the 
expression of GLT-1 was found [6,27], but occasionally a decreased GLAST [27] and GLT-1 [28] expression was demonstrated. Thus, it is obvious that a decreased expression of the neuronal glutamate transporter EAAC1 is not compensated for by changing the expression of the glial transporters GLAST and GLT-1, which is in favor of the hypothesis that the change in EAAC1 is by itself a compensatory mechanism.

To the best of our knowledge, no papers have been published that investigated glutamate transporter expression after HT-induced experimental FS. It has been previously shown that hyperexcitability occurs after FS in these animals, and that later in life spontaneous seizures develop in one third of the animals $[20,21]$. No changes in hippocampal expression of GLAST, GLT-1 and EAAC1 after FS were found in the current study, as measured by area fraction (i.e. number of immunoreactive cells) and mean gray value (i.e. total expression). This was somewhat surprising since we previously showed that less newborn DGCs express EAAC1 [23] and that the number of EAAC1 expressing pyramidal cells is decreased 8 weeks after FS in CA3 (unpublished results). A possible explanation might be the following (see fig. 7): first, FS induce acute cell death in the hippocampus. This was concluded in an earlier study since more newborn cells were found in the dentate gyrus, but neuronal density was unaltered, suggesting a turnover instead of an increased number of DGCs [22]. Furthermore, cell damage in CA3, measured by a silver staining, was increased (unpublished results). Then, because of this loss of EAAC1 expressing neurons, the remaining cells increase their expression of EAAC1 as a compensatory mechanism. Finally, in the case of the newborn DGCs, when they differentiate, less express EAAC1, to ultimately regain normal levels of EAAC1 expression in the hippocampus. This might also explain why GLAST and GLT-1 levels were not affected, because, as suggested earlier, a change in EAAC1 expression is already by itself a compensatory mechanism. Future studies, in which EAAC1 expression is measured per individual cell (i.e. making a distinction between newborn and remaining cells), and at different time points after FS, may elucidate this issue. 


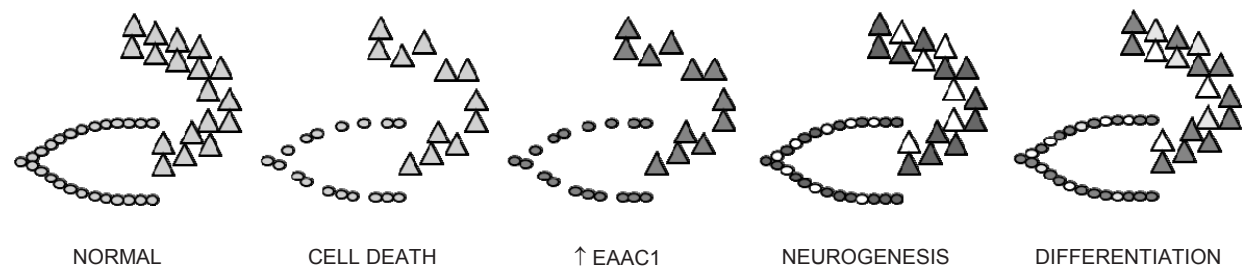

FIG. 7. Schematic representation of the hypothetical change in EAAC1 expression after hyperthermia-induced seizures. Circles $=$ dentate granule cells; Triangles= pyramidal cells of CA3.

\section{Blocking glutamate transport with TBOA did not induce behavioral or electrographic seizures}

As mentioned above, we previously found that less newborn DGCs were expressing EAAT3 [23] after FS compared to controls. It was therefore hypothesized that this causes a state of hyperexcitability in adulthood, and that pharmacologically blocking glutamate transport with TBOA can induce seizure activity in FS animals. However, no behavioral or electrographic seizures were observed after TBOA injection. Other studies have shown that TBOA is able to induce seizures in immature rats [17,18], as well as in adult rats [31] where TBOA also increased the extracellular glutamate concentration as measured using microdialysis. Then why did we not observe seizures after administering TBOA to adult rats that had had early-life FS? One reason might be the method of TBOA administration. Montiel et al. [31] injected TBOA directly into the hippocampus, in contrast to the present study in which TBOA was injected into the tail vein. Similar to our results, Shimamoto et al. [32] injected adult rats intraperitoneally with TBOA and did not find an effect. They concluded that TBOA was unable to cross the blood-brain barrier (BBB), thus to overcome this problem, we injected TBOA together with mannitol. Mannitol is often used in the clinic as an osmotic agent for the management of cerebral edema, but by opening of the BBB, it can also facilitate the uptake of substances into the brain. Artificial opening of the BBB with mannitol has been used in other animal models of epilepsy [33] and the permeability is usually reversed within 10 minutes [34]. It is therefore unlikely that the recording period of 30 minutes 
after TBOA injection was not long enough and that seizures occurring later have been missed. The dose of TBOA was considered to be relatively low and unable to induce seizures in control rats, and we hypothesized that FS animals would react to this dose. This assumption was based on the reduced number of EAAC1 expressing newborn DGCs that was previously found [23]. However, since the overall expression of the glutamate transporters, as shown in the current study, was not changed by FS, this is therefore the most plausible explanation for the lack of seizure activity after TBOA injection.

Evidence that TBOA did enter the brain came from the observed decrease in glutamate transporter expression in TBOA-treated NT and $\mathrm{HT}+$ animals. However, we cannot confirm if this was due to TBOA itself, mannitol, or the combination mannitol-TBOA since we did not include a vehicle (mannitol) group. Neurotoxicity due to TBOA is well known [35]. It is therefore possible to assume that TBOA crossed the BBB and induced cell death, thereby decreasing the expression of the hippocampal glutamate transporters.

\section{Conclusion}

According to the results of the present study, it is highly unlikely that changes in glutamate transport are related to the hyperexcitability previously found to exist after FS. 


\section{References}

1. Nicholls, D., Attwell, D., The release and uptake of excitatory amino acids. Trends Pharmacol. Sci., 1990, 11(11), 462-468.

2. Danbolt, N.C., Glutamate uptake. Prog. Neurobiol., 2001, 65(1), 1-105.

3. During, M.J., Spencer, D.D., Extracellular hippocampal glutamate and spontaneous seizure in the conscious human brain. Lancet, 1993, 341(8861), 1607-1610.

4. Petroff, O.A., et al., Glutamate-glutamine cycling in the epileptic human hippocampus. Epilepsia, 2002, 43(7), 703-710.

5. Mathern, G.W., et al., Hippocampal GABA and glutamate transporter immunoreactivity in patients with temporal lobe epilepsy. Neurology, 1999, 52(3), 453-472.

6. Crino, P.B., et al., Increased expression of the neuronal glutamate transporter (EAAT3/EAAC1) in hippocampal and neocortical epilepsy. Epilepsia, 2002, 43(3), 211-218.

7. Proper, E.A., et al., Distribution of glutamate transporters in the hippocampus of patients with pharmaco-resistant temporal lobe epilepsy. Brain, 2002, 125(1), 32-43.

8. Hoogland, G., et al., Synaptosomal glutamate and GABA transport in patients with temporal lobe epilepsy. J. Neurosci. Res., 2004, 76(6), 881-890.

9. Tessler, S., et al., Expression of the glutamate transporters in human temporal lobe epilepsy. Neuroscience, 1999, 88(4), 1083-1091.

10. Meldrum, B.S., Akbar, M.T., Chapman, A.G., Glutamate receptors and transporters in genetic and acquired models of epilepsy. Epilepsy Res., 1999, 36(2-3), 189-204.

11. Rothstein, J.D., et al., Knockout of glutamate transporters reveals a major role for astroglial transport in excitotoxicity and clearance of glutamate. Neuron, 1996, 16(3), 675-686.

12. Sepkuty, J.P., et al., A neuronal glutamate transporter contributes to neurotransmitter GABA synthesis and epilepsy. J. Neurosci., 2002, 22(15), 6372-6379.

13. Watanabe, T., et al., Amygdala-kindled and pentylenetetrazole-induced seizures in glutamate transporter GLAST-deficient mice. Brain Res., 1999, 845(1), 92-96.

14. Peghini, P., Janzen, J., Stoffel, W., Glutamate transporter EAAC-1-deficient mice develop dicarboxylic aminoaciduria and behavioral abnormalities but no neurodegeneration. Embo. J., 1997, 16(13), 3822-3832.

15. Tanaka, K., et al., Epilepsy and exacerbation of brain injury in mice lacking the glutamate transporter GLT-1. Science, 1997, 276(5319), 1699-1702.

16. Campbell, S., Hablitz, J.J., Modification of epileptiform discharges in neocortical neurons following glutamate uptake inhibition. Epilepsia, 2005, 46(Suppl. 5), 129-133.

17. Demarque, M., et al., Glutamate transporters prevent the generation of seizures in the developing rat neocortex. J. Neurosci., 2004, 24(13), 3289-3294.

18. Milh, M., et al., Inhibition of glutamate transporters results in a "suppression-burst" pattern and partial seizures in the newborn rat. Epilepsia, 2007, 48(1), 169-174. 
19. Cendes, F., et al., Early childhood prolonged febrile convulsions, atrophy and sclerosis of mesial structures, and temporal lobe epilepsy: MRI volumetric study. Neurology, 1993, 43(6), 1083-1087.

20. Dube, C., et al., Prolonged febrile seizures in the immature rat model enhance hippocampal excitability long term. Ann. Neurol., 2000, 47(3), 336-344.

21. Dube, C., et al., Temporal lobe epilepsy after experimental prolonged febrile seizures: prospective analysis. Brain, 2006, 129(4), 911-922.

22. Lemmens, E.M., et al., Gender differences in febrile seizure-induced proliferation and survival in the rat dentate gyrus. Epilepsia, 2005, 46(10), 1603-1612.

23. Lemmens, E.M., et al., Cytogenesis in the dentate gyrus after neonatal hyperthermia-induced seizures: What becomes of surviving cells? Epilepsia, 2008, 49(5), 853-860.

24. Baram, T.Z., Gerth, A., Schultz, L., Febrile seizures: an appropriate-aged model suitable for long-term studies. Brain Res. Dev. Brain Res., 1997, 98(2), 265-270.

25. Paxinos, G., Watson, C., The Rat Brain in Stereotaxic Coordinates. 4th ed. 1998, San Diego, $\mathrm{CA}$, Academic Press.

26. Bradford, H.F., Glutamate, GABA and epilepsy. Prog. Neurobiol., 1995, 47(6), 477-511.

27. Miller, H.P., et al., Alterations in glutamate transporter protein levels in kindling-induced epilepsy. J. Neurochem., 1997, 68(4), 1564-1570.

28. Ueda, Y., et al., Collapse of extracellular glutamate regulation during epileptogenesis: downregulation and functional failure of glutamate transporter function in rats with chronic seizures induced by kainic acid. J. Neurochem., 2001, 76(3), 892-900.

29. Gorter, J.A., et al., Glutamate transporters alterations in the reorganizing dentate gyrus are associated with progressive seizure activity in chronic epileptic rats. J. Comp. Neurol., 2002, 442(4), 365-377.

30. Zhang, G., et al., Long-term alterations in glutamate receptor and transporter expression following early-life seizures are associated with increased seizure susceptibility. J. Neurochem., 2004, 88(1), 91-101.

31. Montiel, T., et al., Differential effects of the substrate inhibitor I-trans-pyrrolidine-2,4dicarboxylate $(\mathrm{PDC})$ and the non-substrate inhibitor DL-threo-beta-benzyloxyaspartate (DLTBOA) of glutamate transporters on neuronal damage and extracellular amino acid levels in rat brain in vivo. Neuroscience, 2005, 133(3), 667-678.

32. Shimamoto, K., et al., Characterization of novel L-threo-beta-benzyloxyaspartate derivates, potent blockers of the glutamate transporters. Mol. Pharmacol., 2004, 65(4), 1008-1015.

33. van Vliet, E.A., et al., Blood-brain barrier leakage may lead to progression of temporal lobe epilepsy. Brain, 2007, 130(2), 521-534.

34. Rapoport, S.I., Osmotic opening of the blood-brain barrier: principles, mechanism, and therapeutic applications. Cell. Mol. Neurobiol., 2000, 20(2), 217-230.

35. Guiramand, J., et al., Gliotoxicity in hippocampal cultures is induced by transportable, but not by nontransportable, glutamate uptake inhibitors. J. Neurosci. Res., 2005, 81(2), 199-207. 


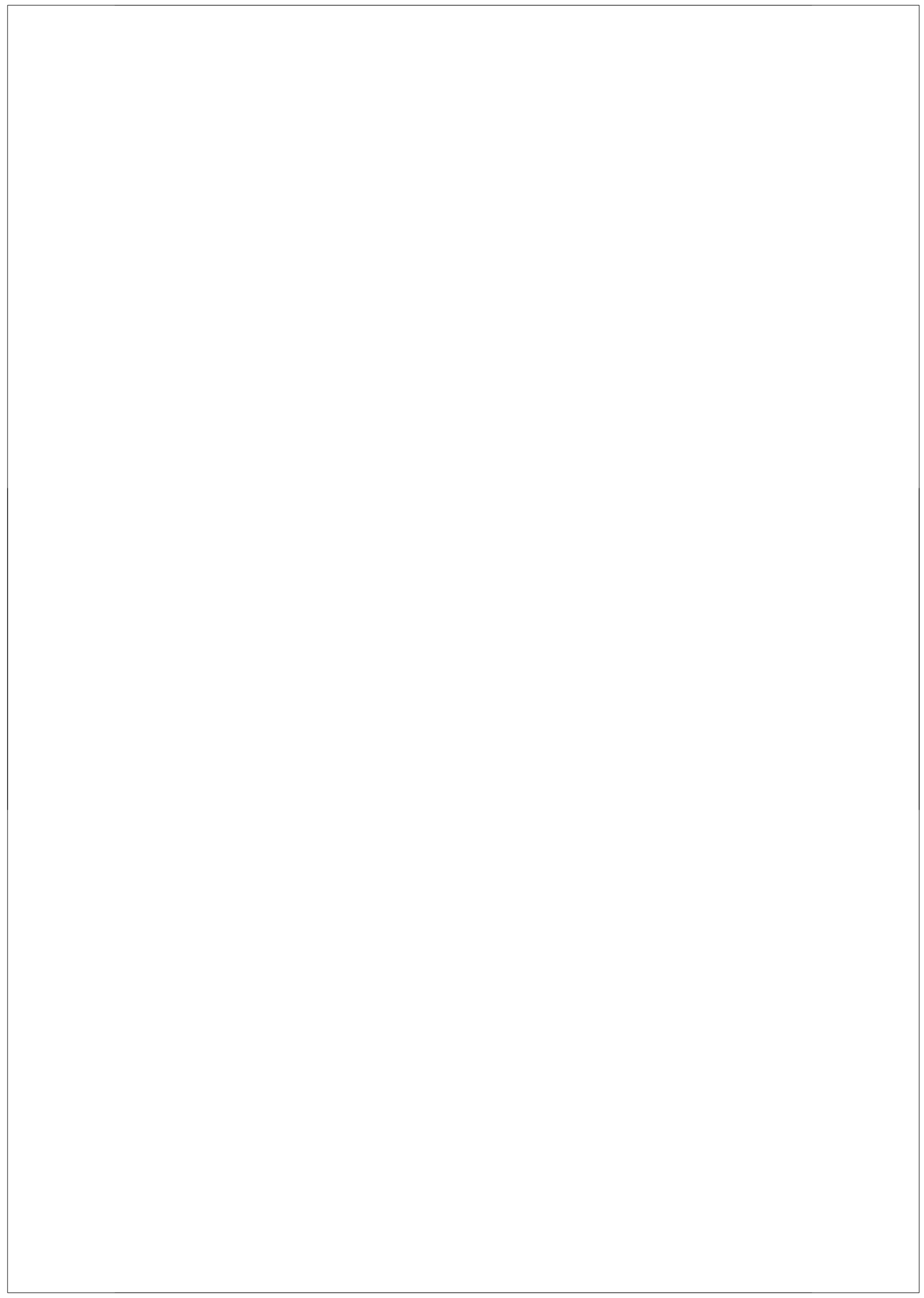




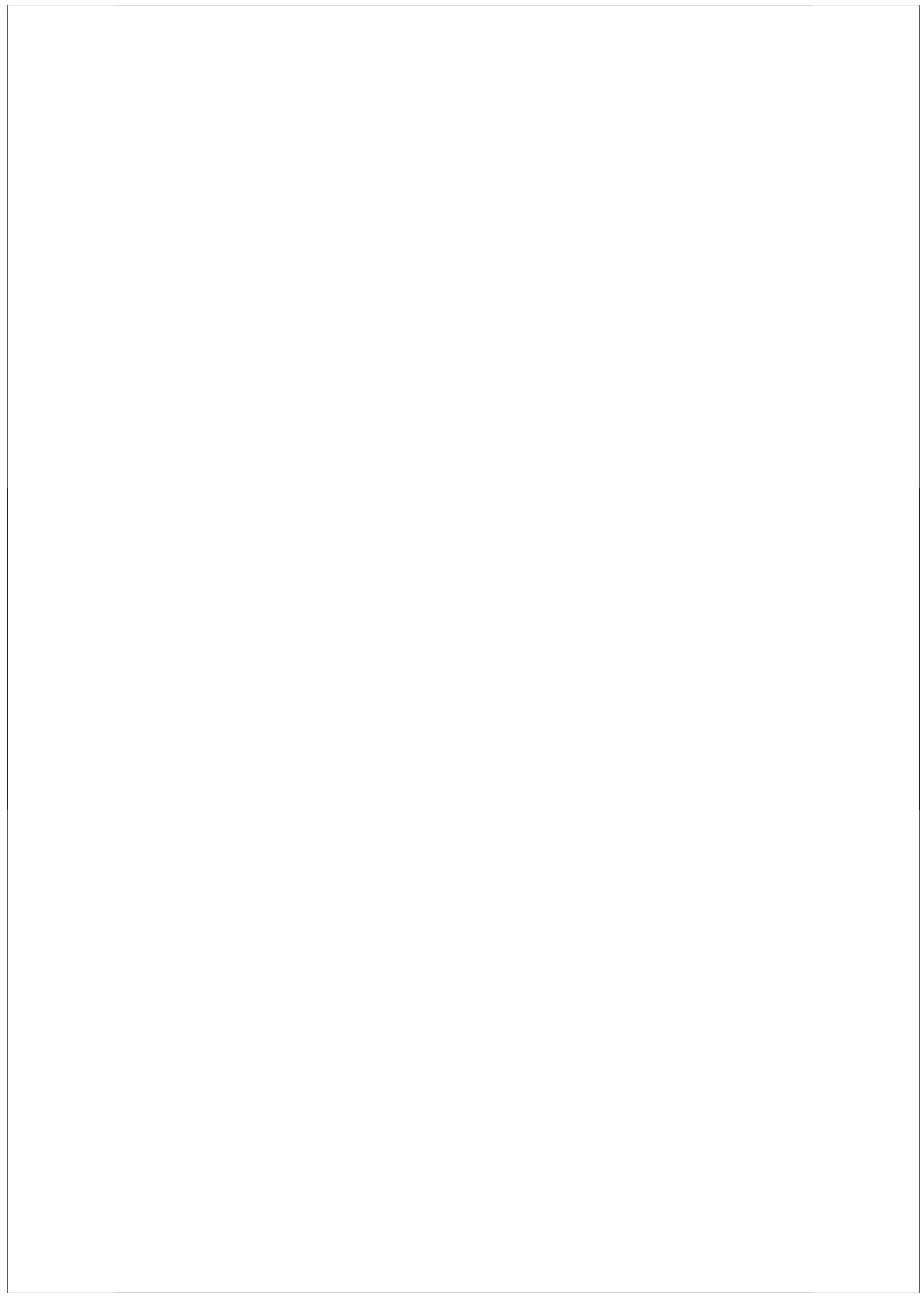



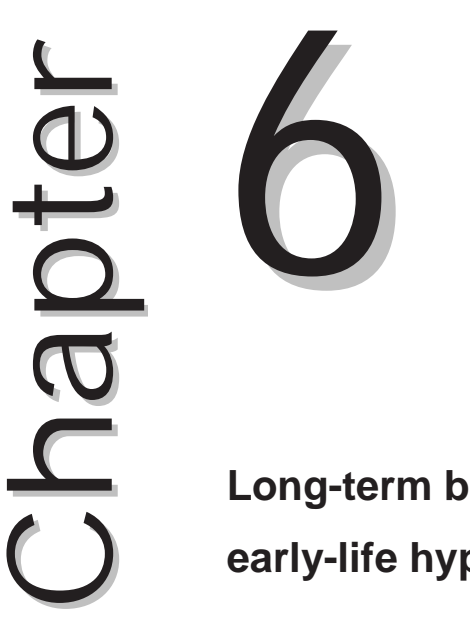

\section{Long-term behavioral outcome after} early-life hyperthermia-induced seizures

Evi M.P. Lemmens*, Brenda Aendekerk*, Olaf E.M.G. Schijns, Arjan Blokland, Emile A.M. Beuls, Govert Hoogland *Authors contributed equally

Epilepsy and Behavior, under review.

ABSTRACT

Febrile seizures (FS) are one of the most common type of seizures in the developing brain. It was suggested that FS cause cognitive deficits that proceed into adulthood, but conflicting results have been reported. The aim of the present study was to examine whether experimental FS can cause long-term cognitive or behavioral deficits.

FS were induced by hyperthermia ( $30 \mathrm{~min}, \sim 41^{\circ} \mathrm{C}$ ) in 10-day old rat pups and behavioral testing was performed. Hippocampal-dependent water maze learning, locomotor activity, and choice-reaction time parameters (e.g. reaction time (RT)) were generally not affected by FS. However, a more detailed analysis revealed that RTs to the right side was slower than to the left in controls while this was not observed after FS.

Early-life experimental FS do not cause overt cognitive and behavioral deficits, which is in line with previous work, but eliminated the lateralization effect in reaction time known to exist in normal controls. 


\section{Introduction}

The consequences of early-life seizures have been a focus of research for many years. Febrile seizures (FS) for example, are thought to play a role in the development of hippocampal sclerosis (HS)-associated temporal lobe epilepsy (TLE) [1], which is often accompanied by memory deficits [2]. Though most clinical studies failed to show adverse effects of FS on cognition [3,4], some indicate that FS can cause long-lasting cognitive defects [5] or can even improve memory performance [6]. In addition, FS have been proposed as an environmental risk factor for attention-deficit hyperactivity disorder (ADHD) and suggested to share a common antecedent with ADHD [7] and autism [8]. A drawback from clinical studies is that they cannot separate the effects of the seizure itself (e.g. seizure type, number, and duration) from that of preexisting brain pathology and treatment. Therefore controversy remains about the effects of early-life FS on long-term cognitive and behavioral outcome.

Animal models offer a more standardized and better controllable study design and can thereby overcome this drawback. Using these models, it was shown that early-life seizures disrupt hippocampal development resulting in longlasting functional changes. Several developmental processes which are affected are also known to be involved in learning, memory and behavior [9]. In this respect, we previously demonstrated that experimental FS change dentate granule cell (DGC) proliferation and survival [10], and DGC phenotype [11]. Not only changes in neurogenesis, but also in the expression of receptors [12,13], ion channels [14-16], and cytokines [17,18], as well as mossy fiber sprouting [19], have been reported to occur after FS. In the current study, we used an animal model of hyperthermia (HT)-induced FS [20] to analyze whether these seizures provoke cognitive deficits (i.e. learning and memory) and changes in diurnal and nocturnal locomotor activity. For that purpose, we used the Morris water maze (MWM) task, an open field setting, and a choice reaction time task in a Skinner box. In addition, we also measured locomotor activity after injecting a subconvulsive dose of kainic acid. 


\section{Materials and Methods}

\section{Induction of hyperthermia}

Male Sprague-Dawley rat pups (Harlan, the Netherlands) were culled to litters of 9-10 rats at postnatal day (PN) 1, and supplied with a foster mother. Home cage facilities included: a reversed $12 \mathrm{~h}$ light/dark cycle ( $\sim 37$ lux lights on from 18:00 till 6:00 hours), $\sim 21^{\circ} \mathrm{C}$ room temperature, background noise provided by a radio, and unlimited access to food and water. HT was induced as described previously [10]. Briefly, on PN10, the pups received $10 \mathrm{ml} 0.9 \%$ saline/kg body weight subcutaneously to prevent dehydration, and were placed in a Perspex cylinder (one pup per cylinder) with a regulated stream of heated air coming from a hair dryer placed $50 \mathrm{~cm}$ above the rats. Core temperatures were measured before and at $2.5 \mathrm{~min}$ intervals during the exposure to heated air. Behavior of the rats was monitored by two observers independently. When the core temperature reached $\geq 39.5^{\circ} \mathrm{C}$, usually within $5 \mathrm{~min}$, the volume and temperature of the air were adjusted in order to maintain a core temperature of $41-42.5^{\circ} \mathrm{C}$ for $30 \mathrm{~min}$. After the $30-\mathrm{min} \mathrm{HT}$ period, rats were cooled in room temperature water to rapidly regain their baseline core temperature, and returned to their home cage. Littermates were used as normothermia (NT) controls and were submitted to the same treatment, except that their core temperature was kept at baseline value $\left(33-35^{\circ} \mathrm{C}\right)$ during their stay in the cylinder. All pups were weaned at PN22, and then housed 2 to 3 per cage. All experimental procedures were approved by the local ethical committee of Maastricht University and complied with governmental laws. Figure 1 indicates the ages at which subsequent behavioral testing was performed. 


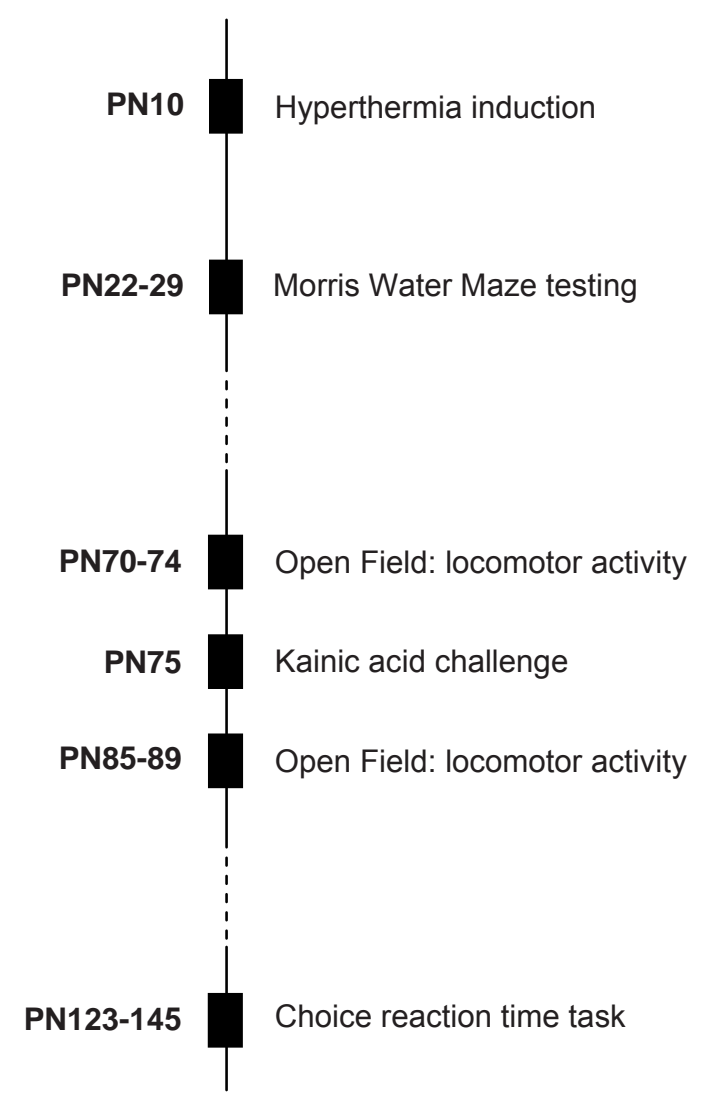

FIG. 1. Scheme indicating the ages at which the behavioral tests were performed. At postnatal day (PN) 10, rats underwent hyperthermia $\left(\sim 41^{\circ} \mathrm{C}\right)$ or normothermia $\left(\sim 35^{\circ} \mathrm{C}\right)$ treatment for $30 \mathrm{~min}$. From PN22-29, learning and memory were tested in a Morris water maze (MWM). Locomotor activity in adulthood was assessed using an open field setting from PN70-89, including a challenge with the convulsant kainate at PN75. Finally, a choice reaction time task was performed from PN123-145 to measure the rat's response to a signal and the processing of that response. Note that different litters of animals were used for the MWM than for the assessment of the locomotor activity and reaction time responding. 


\section{Behavioral testing}

MORRIS WATER MAZE TASK. Twelve days after HT induction, spatial learning of the rats was assessed using a method based on the task described by Morris [21]. Testing was conducted between 9:00 and 11:00, in a room with dimmed light ( $\sim 10$ lux) and background noise provided by a radio. Animals were allowed 30 min habituation to the test room before the test commenced. The test was performed using a circular black pool (1.22 m diameter $\times 0.5 \mathrm{~m}$ height) filled with $\sim 20^{\circ} \mathrm{C}$ tap water. A black platform (11 cm diameter) was located in a fixed position in the west quadrant of the pool at $\sim 21 \mathrm{~cm}$ from the wall, and made invisible by placing it $\sim 2 \mathrm{~cm}$ below the water surface. A trial started by placing the rat into the pool, randomly in one of four locations (north, east, south, or west), facing the perimeter, and was terminated when the rat found the platform. If the rat could not find the platform within $60 \mathrm{~s}$, it was guided to the platform, and placed on the platform for $3 \mathrm{~s}$ before it was removed from the pool. Each rat performed four trials per day during 7 consecutive days. Within a block of four trials, each rat returned to its individual holding cage for $\sim 10 \mathrm{~min}$ before the next trial commenced. Swimming behavior of the rats was recorded by a camera positioned centrally above the pool and connected to an EthoVision tracking system (Noldus Equipment, Wageningen, the Netherlands). The parameters analyzed included: latency (i.e. time to find the platform), distance (i.e. the length of the path swum to the platform), and velocity (i.e. distance divided by latency). The day following the last trial (PN29), a probe trial was given to test whether the rats had actually learned the position of the platform. For this purpose the platform was removed from the pool and the rats were allowed to swim for $30 \mathrm{~s}$. In this trial, the percentage of time the rats spent in the platform quadrant was recorded.

LOCOMOTOR ACTIVITY. Locomotor activity was recorded in an open field arena by a camera connected to an EthoVision tracking system. The parameters analyzed were distance (i.e. the length of the path walked from the 
starting position) and velocity (i.e. distance divided by time). Background noise in the test room was provided by a radio.

Between PN70-74 and between PN85-89, the circadian locomotor activity of each rat was recorded twice from 16:30-21:00 hours ("lights on"; 25 lux) to measure diurnal activity, and twice from 21:00-8:30 hours ("lights off"; $\sim 4$ lux red light) to measure nocturnal activity. For this purpose, four animals were tested simultaneously for which the large open field arena ( $1 \mathrm{~m}$ long $\times 1 \mathrm{~m}$ wide $\times 0.4$ $\mathrm{m}$ high) was divided into 4 small arenas (each $0.5 \times 0.5 \times 0.4 \mathrm{~m}$, with a black floor, two black (inner) and two transparent (outer) walls, and with food and water in opposite corners).

Previously it was shown that HT-induced seizures decrease the threshold for developing kainic acid-induced seizures in adulthood [22]. Therefore, on PN75, about nine weeks after hyperthermia induction, each rat received a kainic acid injection (intraperitoneally, $5 \mathrm{mg} / \mathrm{kg}$ in $0.9 \%$ saline, $\mathrm{pH} 7.4$ ), a dose previously described to be subconvulsive in naïve animals [22,23]. Immediately following kainate administration, the rat was placed in the large open field arena, with a black floor and transparent walls. For $30 \mathrm{~min}$, behavior was monitored by two observers, and motor activity was recorded. This was conducted between 10:00 and 16:00 hours in a room with dimmed light ( 10 lux), and background noise provided by a radio. Hereafter rats returned to their home cages. As a baseline, and to measure possible effects of the kainic acid on circadian activity, each rat was assessed for an additional 30 minutes per day in the large open field arena between PN70-74 and PN85-89.

CHOICE REACTION TIME TASK. From PN108 till PN123, the time necessary to respond to an auditory and visual cue was tested in a Skinner box using a choice reaction time (CRT) task as previously described by Blokland [24]. Food deprivation to $85 \%$ of free feeding weight was maintained throughout testing for motivation. First, rats were trained to learn the task. They learned to push a hinged panel to retrieve a food reward ( $45 \mathrm{mg}$ food pellets). During the next stage of training, the rats had to push the hinged panel for a randomized variable hold duration of $0.5-1.0 \mathrm{~s}$ (steps of $0.1 \mathrm{~s}$ ). When the hold duration had 
elapsed, an auditory stimulus was presented which was turned off when the rat withdrew its nose from the hinged panel in front of the food tray. A high tone stimulus $(10 \mathrm{kHz}, 80 \mathrm{~dB})$ required pressing the left lever, and a low tone $(2.5 \mathrm{kHz}, 80 \mathrm{~dB})$ required pressing the right lever. Whenever a lever was inserted into the Skinner box, the cue light above that lever was switched on. A food reward was given when the rat had pressed the lever. When a rat did not succeed in pushing the hinged panel for the entire hold duration, a new trial started upon pressing the panel. Rats were subjected daily to two sessions (one at 9:00 and one at 14:00). A session lasted until the rat had completed 60 trials (inter-trial interval= $10 \mathrm{~s}$ ) or after $30 \mathrm{~min}$.

From the second session at $\mathrm{PN} 123$, when all rats showed a stable performance, till PN145, the CRT task was performed by changing the hold duration to randomly vary between $0.6-1.5 \mathrm{~s}$ (steps of $0.1 \mathrm{~s}$ ). After four sessions, the final changes in the test conditions were 1) reduction of reinforcement to $50 \%$ (at random) of the responses, and 2) rats were tested every other day. The parameters analyzed were (1) reaction time (RT), i.e. the latency between the onset of the tone and release of the panel, (2) motor time (MT), i.e. the latency between the release of the panel and the lever press, and (3) premature responses (PR), i.e. a measure of response inhibition, presented by the number of trials the rat released the panel before the hold duration had elapsed. RTs shorter than $100 \mathrm{~ms}$ and longer than $1500 \mathrm{~ms}$ as well as MTs longer than $2 \mathrm{~s}$ were considered non-valid, and thus excluded from statistical analyses. All parameters were expressed as a percentage of day 1.

\section{Statistical analysis}

MWM data were analyzed by first averaging the data per day, and then using repeated measures analysis of variance (ANOVA) and LSD multiple comparison post hoc test to analyze the overall effect of treatment. Group differences at each individual day were evaluated using oneway ANOVA with LSD post-hoc test, as well as the locomotor activity and CRT data. Data are presented as mean \pm SEM, and significance was defined as $p<0.05$. 


\section{Results}

\section{Induction of hyperthermia differently affected rat behavior}

During HT treatment, a subset of rats displayed behavioral seizures (HT+FS group) that were typically characterized by arrest of hyperkinesias, followed by falling on their side or back with body flexion and clonic contractions of the limbs, whereas other rats did not display this stereotyped behavior (HT-FS group). None of the NT rats showed seizure behavior during treatment. Out of 27 rats that underwent $\mathrm{HT}$ treatment, 2 rats died within 24 hours but additional mortality was not observed during the rest of the study.

\section{Behavioral testing}

MORRIS WATER MAZE TASK. Hippocampal-dependent spatial learning was assessed using an MWM task. During the first 7 days of testing (PN22-28; fig. 2A), a gradually decrease in escape latency was observed in all rats $(p<0.001)$, or the time needed to find the hidden platform, but there was no significant effect of treatment so all 3 groups learned to perform the task similarly. During the probe test (PN29; fig. 2B), the percentage of time spent in the platform zone was the same for all groups. 

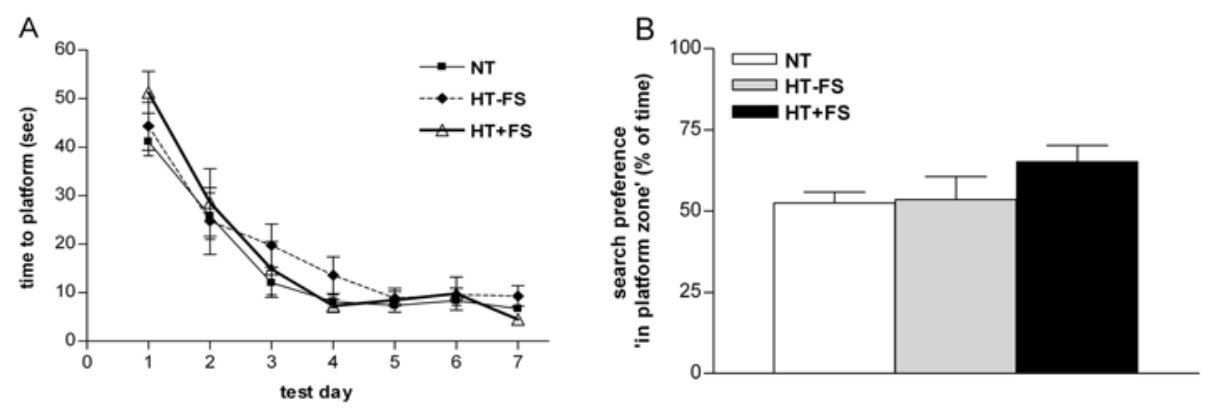

FIG. 2. Morris water maze testing. Hippocampal-dependent learning and memory was tested in a Morris water maze from postnatal day (PN) 22-29. (A) During the first 7 days of testing, the latency (sec) to find the platform decreased over time in all animals, but was not different between the normothermia (NT) controls $(n=12)$, hyperthermia rats without seizures (HT-FS; $(n=7)$ or HT rats with seizures (HT+FS; $(n=6)$. So all rats equally learned to find the escape platform. (B) On the probe trial, the platform was removed and the time spent in the platform quadrant was measured. The results showed that all rats, regardless of the treatment they had received (NT or HT), had a similar search preference so they all spent the same amount (\%) of time in the platform zone.

LOCOMOTOR ACTIVITY. Locomotor activity was acquired for $30 \mathrm{~min} /$ day, 4 consecutive days before, immediately following, and 4 consecutive days after kainate treatment (fig. 3A). Before and after kainate, there was no significant difference in the distance moved between the 3 groups. Also the longer diurnal (fig. 3C) and nocturnal (fig. 3D) registrations showed that all three groups exhibited a similar amount of activity. However, immediately following kainate administration, a significantly decreased locomotor activity was observed in HT+FS and NT, but not in HT-FS animals. Further analysis showed that this decrease could be attributed to the increase in activity in the HT-FS group during the first 15 min after administration, as well as a decrease in activity in the NT and HT+FS group during the second 15 min (fig. 3B). None of the rats in either group showed behavioral seizures during the trials. 

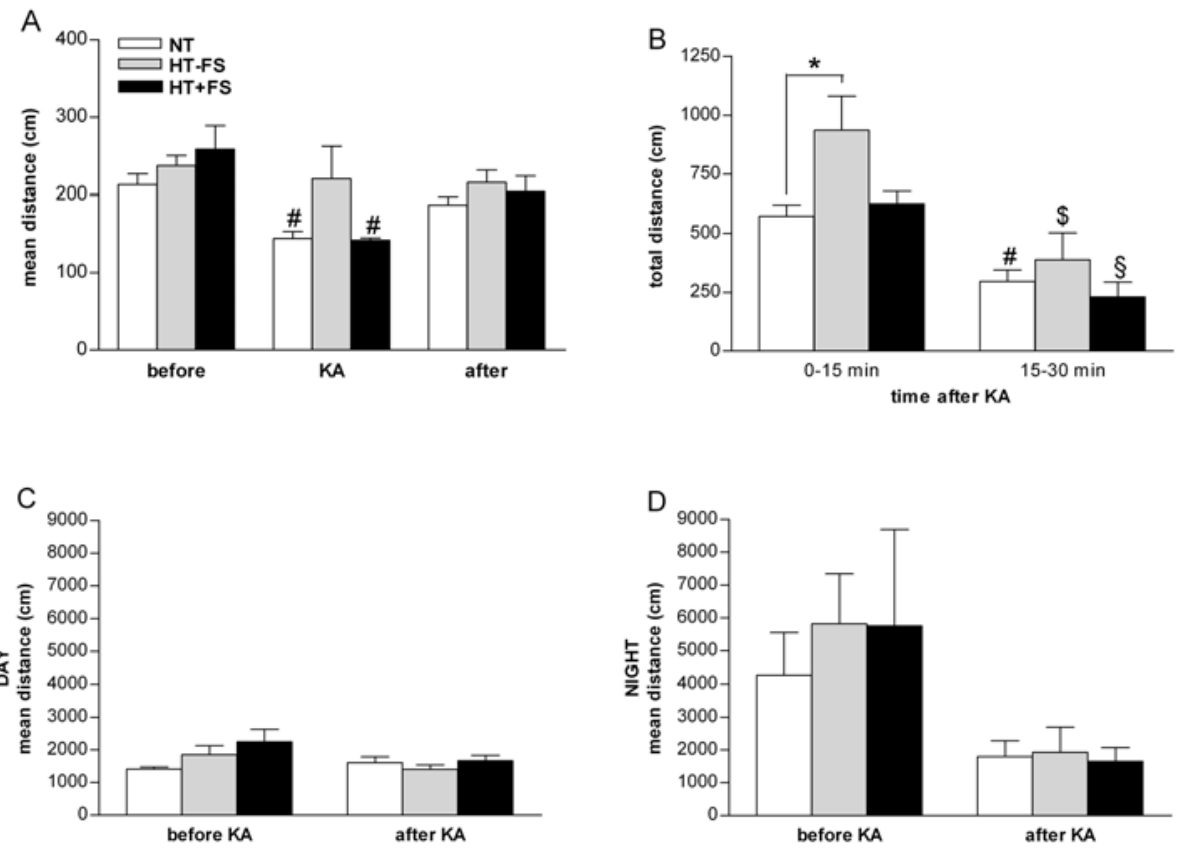

FIG. 3. Locomotor activity. Locomotor activity (mean distance/30 min) was compared between normothermia (NT) control rats $(n=8)$, hyperthermia rats that did not develop seizures (HT-FS; $n=7$ ), and $H T$ rats with seizures $(H T+F S ; n=4)$. Mean distance was not different between the 3 groups throughout daytime before and after injection of kainate, not only when comparing activity during the 30 min trials (A), but also when comparing activity measured for 4.5 hours $(C)$, nor was it different during nighttime (D). Injection of kainate however, did significantly decrease activity in NT and $\mathrm{HT}+\mathrm{FS}$ rats (\# p 0.05), but not in HT-FS rats, compared to before (PN70-74) and after kainate PN85-89) (A). When looking into detail what distance the rats moved during the $30 \mathrm{~min}$ after kainate injection (B), activity was higher during the first $15 \mathrm{~min}$ than during the last $15 \mathrm{~min}$ for the NT control group (\# $p<0.05)$, HT-FS group $(\$ p<0 / 05)$ and $H T+F S$ group $(\S p<0.05)$. Especially activity during the last quarter seemed responsible for the difference in activity between the sessions before, during, and after kainate injection. Also note that HT-FS rats were significantly more active during the first quarter than NT rats but not different from HT+FS rats $\left({ }^{*} p<0.05\right)$. 

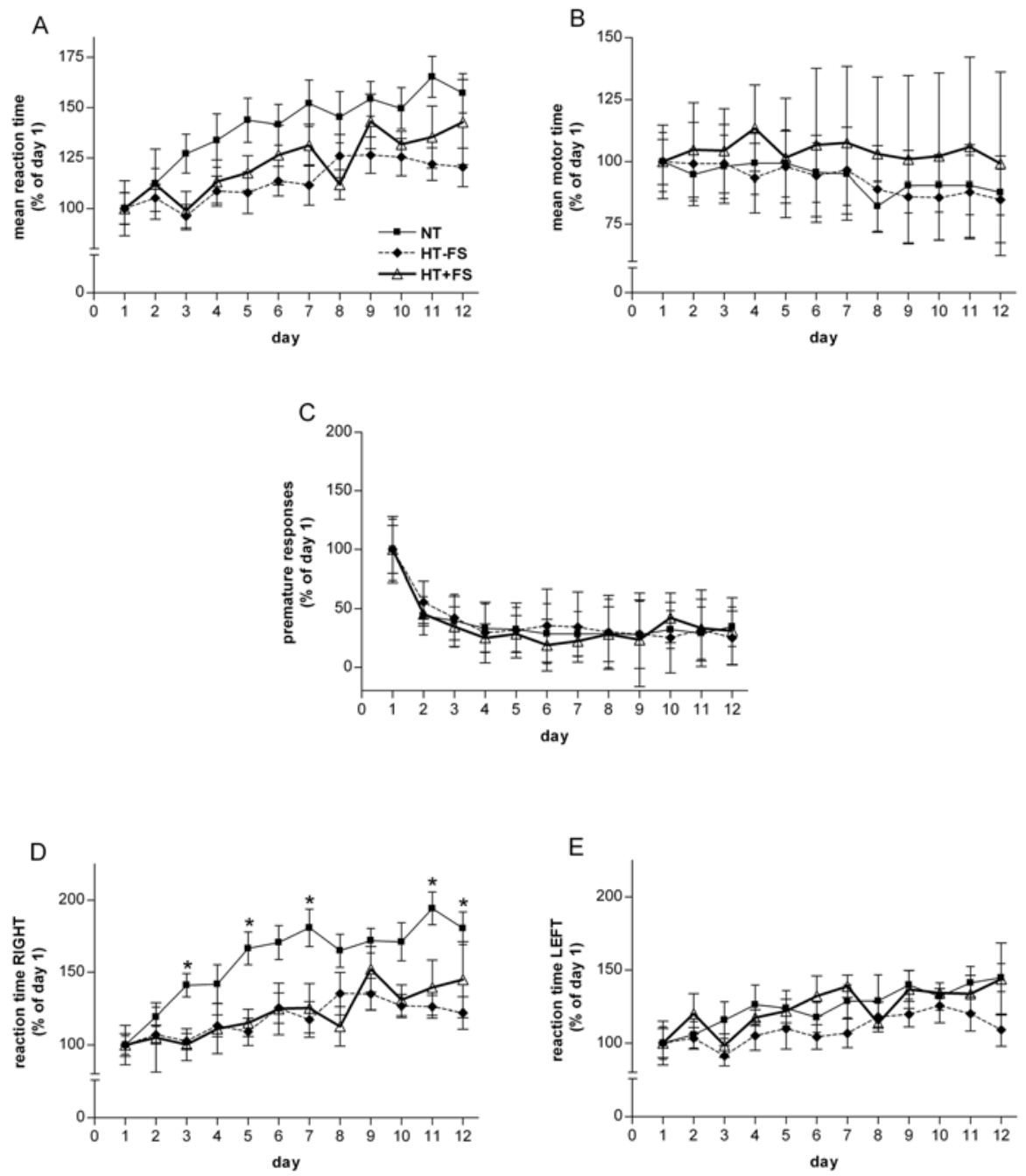

FIG. 4. Performance in a choice reaction time task. Mean reaction time (A), motor time (B), as well as the number of premature responses $(C)$ was found not to be different between normothermia (NT) controls $(n=8)$, hyperthermia rats without seizures (HT-FS; $n=7$ ) and HT rats with seizures $(H T+F S ; n=4)$. Though reaction times to the left were similar between the 3 groups $(E)$, reaction times to the right $(D)$ were faster in HT-FS rats $\left({ }^{*} p<0.05\right.$ at day $3,5,7,11$, and 12$)$ and HT+FS rats $\left({ }^{*} p<0.05\right.$ at day 3 and 11$)$. 
CHOICE REACTION TIME TASK. RTs (fig. 4A), MTs (fig. 4B) and the number of PRs (fig. 4C), were not different between the 3 treatment groups at any day during the 12-day CRT that was performed 4 months after HT treatment. In all 3 groups however, did RTs increase over the test days $(p<0.01)$, while MTs remained stable. The number of PRs decreased during the first few days of testing and was steady thereafter. To investigate a possible lateralization effect, overall RTs were separated into right (fig. 4D) and left (fig. 4E) RTs. Left RTs were not different between the groups, while right RTs were higher in NT controls than in HT-FS rats ( $p<0.05$ on test day $3,5,7,11$, and 12) and HT+FS rats $(p<0.05$ on test day 3 , and 11). When comparing RTs to the left and right for each individual animal, all control rats showed lateralization with higher reaction times to the right than to the left. In contrast, HT-FS and HT+FS did not show this lateralization pattern (fig. $5 A-C$ ).
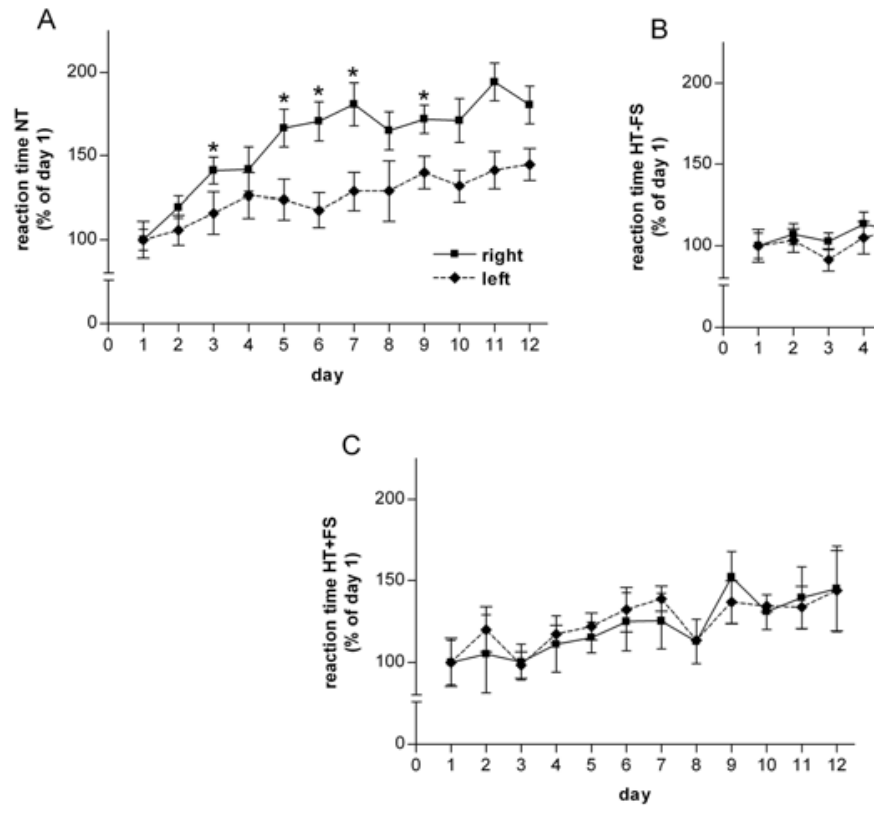

FIG. 5. Lateralization of reaction times. When comparing reaction times to the left and right for each individual group, all normothermia $(N T)$ controls $(n=8)$ showed lateralization with higher reaction times to the right than to the left $\left({ }^{*} p<0.05\right)(A)$. In contrast, hyperthermia rats without seizures ( $B$; $\mathrm{HT}-\mathrm{FS} ; \mathrm{n}=7)$ and $\mathrm{HT}$ rats with seizures $(\mathrm{C} ; \mathrm{HT}+\mathrm{FS} ; \mathrm{n}=4)$ did not show this lateralization pattern. 


\section{Discussion}

The most important findings of the present study are that early-life experimental FS (1) do not affect hippocampal-dependent learning, nor (2) diurnal and nocturnal locomotor activity, or (3) performance in a CRT task. These data further suggest that early-life FS can have a lasting effect on the lateralization of reaction time performance.

The effects of seizures on cognitive and behavioral functioning have been studied extensively in adults. Cognitive deficits in epilepsy patients have often been attributed to the effect of antiepileptic drugs (AEDs), but evidence exists that this is not always the case. Indeed, AEDs, especially polytherapy, can worsen cognitive outcome, but the seizures, the seizure etiology, and especially the epileptic processes themselves (e.g. changes in ion channel expression) are emerging ever more as the culprits. The notion that early-life seizures cause little cognitive or behavioral deficits has come from population studies $[3,4,25,26]$ but is currently under revision. To what extent seizures may affect long-term outcome is probably dependent on the stage of brain development during which the seizures occur.

The current study investigated effects of early-life seizures on cognitive and behavioral outcome in an animal model of FS, and showed that there were no adverse effects on hippocampal-dependent learning and locomotor activity in adulthood. This observation is in agreement with other studies that have used the same animal model. Chang et al. [27] found that memory deficits are not present after a single FS episode, but that more than 3 seizures episodes cause long-term impairment in the MWM task. This was not accompanied by cell loss and mossy fiber sprouting. Mesquita et al. [28] also failed to show memory deficits in the MWM or changes in locomotor activity. Instead, they found that HT+FS rats developed hyperanxiety in adulthood, which is similar to the findings of Saucier et al. [29]. Werboff and Havlena [30] used a microwave to induce FS in 3-day old rat pups and also failed to show differences in MWM 
learning and locomotor behavior (i.e. activity-wheel performance) at PN30. Similar observations were made by Kornelson et al. [31] who evoked a single HT-induced seizure at PN1,10, or 21 and measured locomotor activity and spatial learning in adulthood. However, the effects of seizures on memory seem not only age-dependent, but also model-specific. For instance, kainic acid administered to rat pups can elicit memory deficits and increase anxiety a few months later $[32,33]$. The older the rats at the time point of seizure induction, the poorer their memory performance, the more anxious they were, and the more neurodegeneration was found. In contrast, Koh and colleagues [23] induced seizures by kainic acid at PN15 and/or PN45, and tested the rats in a MWM at PN50. Rats that experienced seizures only at PN15 did not have memory deficits compared to controls (no seizures), while rats that had seizures at PN45 did not perform well in the MWM, but still better than animals that had seizures both at PN15 and PN45. Similarly, Stafstrom et al. [34] evoked a single seizure by kainic acid in rats at PN5, 10, 20,30, or 60 which had no result in PN5-10 rats, caused mild impairment in the MWM in PN20-30 rats, with the PN30 rats showing slight hyperactivity and increased aggression as well, and induced severe learning and behavioral impairment in PN60 rats. Sarkisian et al. [35] induced multiple KA-seizures in young (PN20-26) and adult (PN60-66) rats, and also found important learning deficits only in the adult rats. They attributed this to the severity of the seizures that was much higher in the adult than in the young rats. For flurothyl-induced seizures, also the age at which seizures are induced as well as the number of seizures are related to later learning impairment and hypoactivity [36-38]. To conclude, seizures in immature animals generally do not cause cognitive or behavioral impairment, while seizures in adults are able to induce adverse effects.

The biological mechanisms of cognitive and behavioral disturbances after seizures are not fully understood. One possibility by which cognitive impairment was thought to arise, is seizure-induced cell death. However, this does not seem to play a major role in cognitive outcome after early-life seizures since the developing brain is found to be relatively resistant to neuronal damage despite 
the low threshold for developing seizures. Alternative explanations may come from seizure-induced mossy fiber sprouting and neurogenesis, but neither one has been found to correlate to cognitive impairment. Yet, as the previously mentioned studies prove, factors such as seizure frequency and thus seizure duration, are able to influence behavioral outcome. Most promising are the data from studies that investigated altered synaptic plasticity as a possible cause of cognitive impairment. Seizure activity and long-term potentiation, the biological substrate of memory, are similar forms of synaptic plasticity. Both involve changes in glutamate release and NMDA receptor expression and activation $[39,40]$. In this respect, Chang et al. [27] have recently shown that phosphorylation of cAMP response-element binding, an important step in the cascade of events leading to long-term memory, is decreased after repetitive FS, but not after a single FS.

Previously, it was shown that HT-induced FS cause hyperexcitability and thereby lower the threshold for inducing seizures in adults [22]. More recently however, Chang et al. were unable to confirm these results [27]. In the present study, the HT+FS rats also failed to develop behavioral seizures when challenged with a subconvulsive dose of kainic acid. It is impossible to exclude subclinical seizures since EEG was not used to detect abnormal brain activity. Another possibility for the discrepancy between the present study and that of Dube et al. [22] is the duration of the HT seizures. They reported FS with a mean duration of more than 20 minutes while the rats in this study developed seizures that lasted less than 10 minutes [10].

Animal models of early-life seizures have rarely submitted rats to a CRT in adulthood. A few studies in which pilocarpine was used to induce status epilepticus, showed that these animals were unable to learn the task (i.e. learn to press a pedal to obtain water) [41,42]. Interestingly, we observed a differentiation in RT to the left and right that was different in HT animals than in NT controls. In this respect, Jansky et al. [43] compared the occurrence of left versus right HS in TLE patients with a history of FS (TLE+FS) with that in TLE 
patients without a history of FS (TLE-FS). They found significantly more cases with right-sided $\mathrm{HS}$ in the TLE+FS patient group and significantly more cases with left-sided HS in the TLE-FS patient group. To the best of our knowledge there are no reports of lateralized hippocampal damage or RT performance in animal models of early-life seizures. Cognitive processes in the brain are known to be lateralized [44]. It has been demonstrated in humans that RTs increase across testing while the number of errors made remains stable, similar to what has been found in the present study, and this is accompanied by decreased blood flow in the left thalamus and cortical regions of the right hemisphere. It was suggested that these changes underlie a shift from intense to automatic attentional processing [45]. The mechanisms for HT treatment to eliminate the lateralization effect found in controls in the present study, are unidentified. When resolved, they might give a hint about subtle lateralized changes that occur after FS.

To conclude, experimental hyperthermia-induced febrile seizures do not cause overt cognitive and behavioral deficits. However, subtle changes were detected, that might become more important later in life. 


\section{References}

1. Cendes, F., et al., Early childhood prolonged febrile convulsions, atrophy and sclerosis of mesial structures, and temporal lobe epilepsy: an MRI volumetric study. Neurology, 1993, 43(6), 1083-1087.

2. Leritz, E.C., Grande, L.J., Bauer, R.M., Temporal lobe epilepsy as a model to understand human memory: the distinction between explicit and implicit memory. Epilepsy Behav., 2006, 9(1), 1-13.

3. Ellenberg, J.H., Nelson, K.B., Febrile seizures and later intellectual performance. Arch. Neurol., 1978, 35(1), 17-21.

4. Verity, C.M., Greenwood, R., and Golding, J., Long-term intellectual and behavioral outcomes of children with febrile convulsions. N. Engl. J. Med., 1998, 338(24), 1723-1728.

5. Kolfen, W., Pehle, K., and Konig, S., Is the long-term outcome of children following febrile convulsions favorable? Dev. Med. Child Neurol., 1998, 40(10), 667-671.

6. Chang, Y.C., et al., Working memory of school-aged children with a history of febrile convulsions: a population study. Neurology, 2001, 57(1), 37-42.

7. Pineda, D.A., et al., Environmental influences that affect attention deficit/hyperactivity disorder: study of a genetic isolate. Eur. Child Adolesc. Psychiatry, 2007, 16(5), 337-346.

8. Levisohn, P.M., The autism-epilepsy connection. Epilepsia, 2007, 48(Suppl. 9), 33-35.

9. Holmes, G.L., Effects of seizures on brain development: lessons from the laboratory. Pediatr. Neurol., 2005, 33(1), 1-11.

10. Lemmens, E.M., et al., Gender differences in febrile seizure-induced proliferation and survival in the rat dentate gyrus. Epilepsia, 2005, 46(10), 1603-1612.

11. Lemmens, E.M., et al., Cytogenesis in the dentate gyrus after neonatal hyperthermia-induced seizures: What becomes of surviving cells? Epilepsia, 2008, 49(5), 853-860.

12. Gonzalez Ramirez, M., et al., Hyperthermia-induced seizures modify the GABA(A) and benzodiazepine receptor binding in immature rat brain. Cell. Mol. Neurobiol., 2007, 27(2), 211-227.

13. Han, Y., et al., Successive alterations of hippocampal gamma-aminobutyric acid B receptor subunits in a rat model of febrile seizure. Life Sci., 2006, 78(25), 2944-2952.

14. Brewster, A., et al., Developmental febrile seizures modulate hippocampal gene expression of hyperpolarization-activated channels in an isoform- and cell-specific manner. J. Neurosci., 2002, 22(11), 4591-4599.

15. Brewster, A.L., et al., Formation of heteromeric hyperpolarization-activated cyclic nucleotidegated $(\mathrm{HCN})$ channels in the hippocampus is regulated by developmental seizures. Neurobiol. Dis., 2005, 19(1-2), 200-207. 
16. Chen, K., et al., Persistently modified h-channels after complex febrile seizures convert the seizure-induced enhancement of inhibition to hyperexcitability. Nat. Med., 2001, 7(3), 331 337.

17. Matsuo, M., et al., Increased IL-1 beta production from dsRNA-stimulated leukocytes in febrile seizures. Pediatr. Neurol., 2006, 35(2), 102-106.

18. Vezzani, A., Inflammation and seizures. Epilepsy Curr., 2005, 5(1), 1-6.

19. Bender, R.A., et al., Mossy fiber plasticity and enhanced hippocampal excitability, without hippocampal cell loss or altered neurogenesis, in an animal model of prolonged febrile seizures. Hippocampus, 2003, 13(3), 399-412.

20. Baram, T.Z., Gerth, A., Schultz, L., Febrile seizures: an appropriate-aged model suitable for long-term studies. Brain Res. Dev. Brain Res., 1997, 98(2), 265-270.

21. Morris, R.G.M., Developments of a water-maze procedure for studying spatial learning in the rat. J. Neurosci. Meth., 1984, 11, 47-60.

22. Dube, C., et al., Prolonged febrile seizures in the immature rat model enhance hippocampal excitability long term. Ann. Neurol., 2000, 47(3), 336-344.

23. Koh, S., et al., Early-life seizures in rats increase susceptibility to seizure-induced brain injury in adulthood. Neurology, 1999, 53(5), 915-921.

24. Blokland, A., Reaction time responding in rats. Neurosci. Biobehav. Rev., 1998, 22(6), 847864.

25. Chang, Y.C., et al., Neurocognitive attention and behavior outcome of school-age children with a history of febrile convulsions: a population study. Epilepsia, 2000, 41(4), 412-420.

26. Nelson, K.B., Ellenberg, J.H., Prognosis in children with febrile seizures. Pediatrics, 1978, 61(5), 720-727.

27. Chang, Y.C., et al., Febrile seizures impair memory and cAMP response-element binding protein activation. Ann. Neurol., 2003, 54(6), 706-718.

28. Mesquita, A.R., et al., Febrile convulsions in developing rats induce a hyperanxious phenotype later in life. Epilepsy Behav., 2006, 9(3), 401-406.

29. Saucier, D., et al., Neonatal heat-induced convulsions affect behaviours in neonatal, juvenile and adult rats, in Advances in Behavioral Biology, M.E. Corcoran and S.L. Moshe, Editors. 2005, Springer. p. 26-36

30. Werboff, J., Havlena, J., Febrile Convulsions in Infant Rats, and Later Behavior. Science, 1963, 142, 684-685.

31. Kornelsen, R.A., et al., The effects of a single neonatally induced convulsion on spatial navigation, locomotor activity and convulsion susceptibility in the adult rat. Brain Res., 1996, 706(1), 155-159.

32. Lynch, M., et al., Long-term consequences of early postnatal seizures on hippocampal learning and plasticity. Eur. J. Neurosci., 2000, 12(7), 2252-2264.

33. Sayin, U., Sutula, T.P., Stafstrom, C.E., Seizures in the developing brain cause adverse longterm effects on spatial learning and anxiety. Epilepsia, 2004, 45(12), 1539-1548. 
34. Stafstrom, C.E., et al., Age-dependent cognitive and behavioral deficits after kainic acid seizures. Epilepsia, 1993, 34(3), 420-432.

35. Sarkisian, M.R., et al., Multiple kainic acid seizures in the immature and adult brain: ictal manifestations and long-term effects on learning and memory. Epilepsia, 1997, 38(11), 11571166.

36. Bo, T., et al., Long-term effects of seizures in neonatal rats on spatial learning ability and Nmethyl-D-aspartate receptor expression in the brain. Brain Res. Dev. Brain Res., 2004, 152(2), 137-142.

37. Holmes, G.L., et al., Consequences of neonatal seizures in the rat: morphological and behavioral effects. Ann. Neurol., 1998, 44(6), 845-857.

38. Huang, L., et al., Long-term effects of neonatal seizures: a behavioral, electrophysiological, and histological study. Brain Res. Dev. Brain Res., 1999, 118(1-2), 99-107.

39. Meador, K.J., The basic science of memory as it applies to epilepsy. Epilepsia, 2007, 48(Suppl. 9), 23-25.

40. Reid, I.C., Stewart, C.A., Seizures, memory and synaptic plasticity. Seizure, 1997, 6(5), $351-$ 359.

41. dos Santos, N.F., et al., Epileptogenesis in immature rats following recurrent status epilepticus. Brain Res. Brain Res. Rev., 2000, 32(1), 269-276.

42. Santos, N.F., et al., Multiple pilocarpine-induced status epilepticus in developing rats: a longterm behavioral and electrophysiological study. Epilepsia, 2000, 41(Suppl. 6), S57-63.

43. Janszky, J., et al., Right hippocampal sclerosis is more common than left after febrile seizures. Neurology, 2003, 60(7), 1209-1210.

44. Hugdahl, K., Lateralization of cognitive processes in the brain. Acta Psychol.(Amst.), 2000, 105(2-3), 211-235.

45. Paus, T., et al., Time-related changes in neural systems underlying attention and arousal during the performance of an auditory vigilance task. J. Cogn. Neurosci., 1997, 9(3), 392-408. 


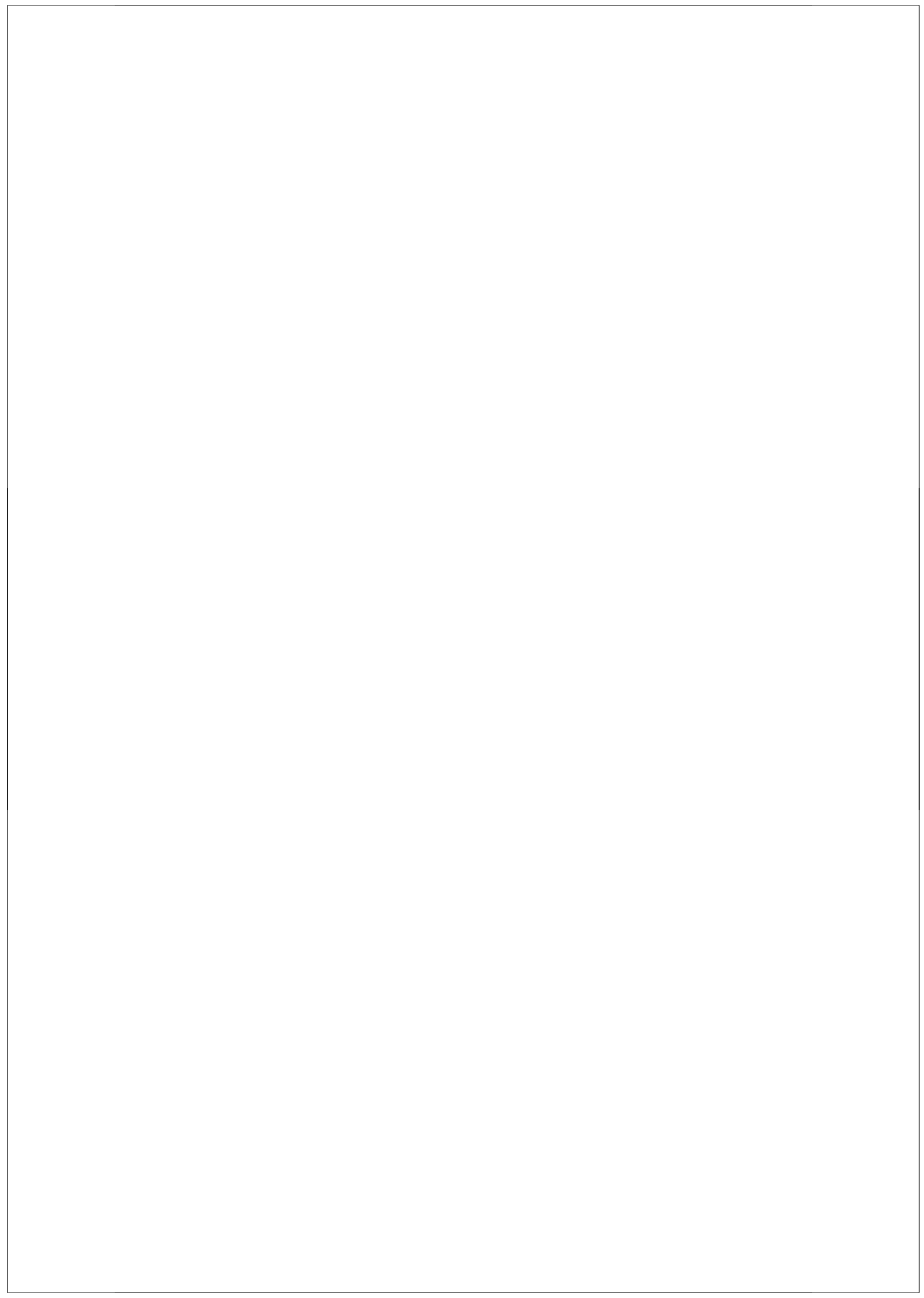




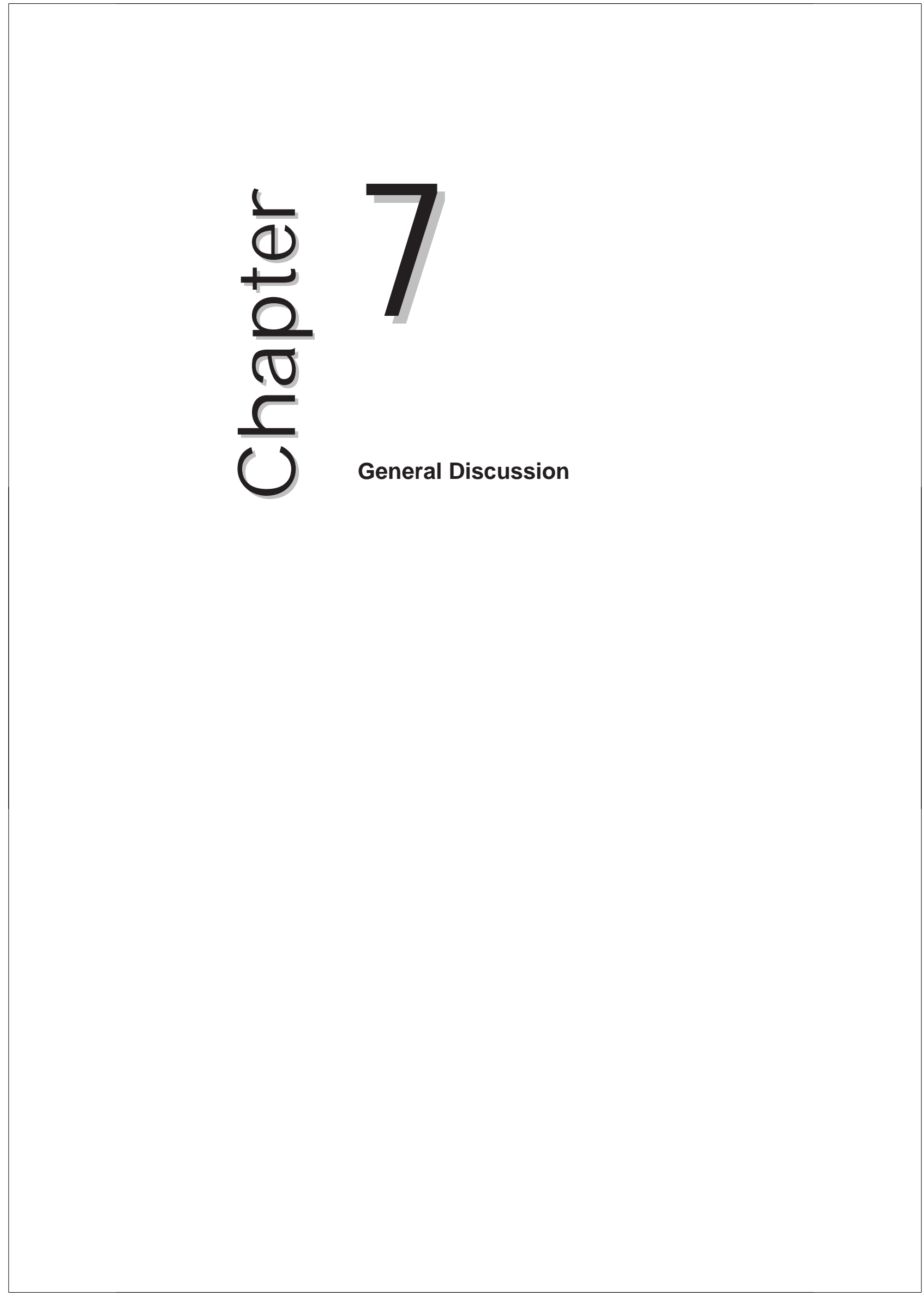


Febrile seizures (FS) early in life, and temporal lobe epilepsy (TLE) associated with hippocampal sclerosis (HS) in adulthood, are thought to be linked. However, the precise nature of the relationship is not well understood. One theory states that FS lead to hippocampal damage that develops into HS and thereby cause spontaneous seizures (i.e. TLE). Evidence for this idea has come from retrospective studies in which they found that HS-TLE patients suffered from FS in childhood more often than TLE patients without HS or controls [1-3]. However, prospective population-based studies failed to show an association between FS and TLE [4,5]. Another theory proposes that preexisting hippocampal pathology, 'the first hit', leads to FS, and that the FS serve as a 'second hit' which then causes HS-associated TLE, or that preexisting pathology predisposes to both FS and TLE with FS being the first expression of TLE. Clinical studies $[6,7]$ have indicated that preexisting pathology is more common in children that develop FS and HS-associated TLE. Also animal studies $[8,9]$ have proven that brain lesions decrease the threshold for FS and increase the probability of developing spontaneous seizures later in life as well as worsen FS-induced damage. In reality, it is not as simple as proposed by any of these theories. It has become more and more clear that TLE is a multifactorial disease with many contributing factors such as genetics, inflammation (i.e. cytokines) and other environmental factors. The fact that there is a latent period between the occurrence of early-life FS and TLE later in life, increases even more the chance that other factors are involved in the disease progression.

\section{Long-term cellular and structural changes induced by FS}

What makes FS even more interesting to study is the fact that they occur during a critical period of brain maturation. Thus, several processes involved in normal brain development such as apoptosis, neurogenesis and synaptogenesis, and the switch from excitation to inhibition, may be affected by FS. It is thought that some of the pathological characteristics of HS (e.g. granule cell dispersion) are 
due to the disturbance of brain developmental processes by FS. We therefore investigated whether FS induce changes in neurogenesis and glutamatergic neurotransmission known to play an important role during development.

By labeling cells that were dividing immediately after hyperthermia- (HT) induced FS, we showed that FS caused an increase in the proliferation and survival rate of cells in the hippocampal dentate gyrus (DG). This resulted in an increased number of newborn cells, mainly neurons at two months after FS, which was gender-specific since males showed this effect but females did not (CHAPTER 2-3). Since some studies indicate that FS are slightly more common in boys than in girls $[10,11]$, this implies that males are also more prone to suffer from the effects of FS. However, the risk of developing recurrent seizures and epilepsy is similar in both genders [12]. Surprisingly, the increased number of newborn cells did not increase the total number of cells in the DG as measured by neuronal density. Thus, it was suggested that FS-induced cell death had occurred but that dying cells were replaced by new cells. We thereby confirmed the results of others that showed that neuronal damage occurs after HT-induced FS without eventually causing cell loss $[13,14]$. Using proton magnetic resonance spectroscopy ( ${ }^{1} \mathrm{H}$-MRS), we found that the level of $\mathrm{N}$-acetylaspartate (NAA) was slightly decreased shortly after FS but was restored two months later (CHAPTER 4). NAA is a reliable marker of neuronal integrity. Thus, the decreased NAA may represent the process of cell death, and subsequently the replacement of lost cells by neurogenesis may cause NAA to increase again to normal levels. Recently, researchers developed a powerful tool for the detection of neural stem cells in vivo, using MRS [15]. In the future, it should be possible to use this method to detect neurogenesis prospectively in humans after experiencing FS and to investigate the relationship between neurogenesis and epileptogenesis.

These data suggest that the role of increased proliferation found after FS is to counteract cellular injury, and thus to act as a neuronal replacement mechanism rather than being a mechanism of hyperexcitability as suggested for TLE 
patients. However, there were already indications from the literature that newborn cells do not simply represent substitutes for the lost cells because these newborn cells were found not to be 'normal'. For example, newborn dentate granule cells (DGCs) remain 'immature', they fail to retract their basal dendrite and thus maintain synaptic contact with excitatory mossy fibers thereby causing hyperexcitability [16]. Furthermore, it has been hypothesized that granule cell dispersion in MTS patients is related to increased neurogenesis and concomitant ectopic location of DGCs and hyperexcitability. No signs of dispersion were seen after HT-induced FS though. Still, neurogenesis may result in hyperexcitability via other manners. In this respect, we determined the expression of the neuronal glutamate transporter EAAC1 in these newborn cells and found it to be decreased two months after FS (CHAPTER 3). Glutamate is the major excitatory neurotransmitter in the brain, and DGCs are mainly glutamatergic. Removal of glutamate from the synaptic cleft is important in protecting cells from excitotoxicity, and recycling of glutamate is necessary for synthesis of the inhibitory neurotransmitter GABA [17]. We therefore hypothesized that this decrease in EAAC1 expressing newborn cells would decrease the threshold for developing seizures in adulthood. However, pharmacological blocking of glutamate transporters did not result in the development of seizure activity in adult rats (CHAPTER 5). Furthermore, when comparing overall hippocampal glial and neuronal glutamate transporter expression, i.e. GLAST, GLT-1, and EAAC1 expression respectively, we failed to show changes in the amount of transporters present after FS. In contrast, vesicular glutamate transporter (VGLUT) levels were recently found to be increased by FS and even further after development of spontaneous FSinduced seizures in adulthood [18]. It is therefore not unlikely that changes in glutamatergic neurotransmission play a major role in FS-induced hyperexcitability and the decreased threshold for developing spontaneous seizures $[19,20]$.

In 2004, a paper was published where they used MRI to investigate whether HT-induced FS cause structural changes similar to those seen in TLE patients 
(i.e. hippocampal atrophy and increases in T2 signal intensity). The rats showed increased signal intensity in different brain structures after FS, but hippocampal damage was not present. Because the follow-up period of 8 days after FS may have been too short and hippocampal atrophy was therefore not yet detectable, we performed a similar study but with a longer follow-up period of 8 weeks and using multimodal MRI to detect subtle (micro)structural changes (CHAPTER 4). Acute FS-induced signal intensity changes were found in the hippocampus but these did not evolve into hippocampal atrophy or gliosis at follow-up. Glutamate and GABA concentrations were similar between FS and control animals, which is not unlikely since glutamate transporter expression was not affected by FS. We concluded that FS cause acute as well as long-lasting effects on brain structure and although no obvious signs of HS were present (e.g. no hippocampal atrophy, gliosis), the microstructural changes that were seen may be an early marker of developing HS. Especially, we were able to detect sprouting after HT-induced FS using multimodal MRI techniques, and confirmed this sprouting using histology (CHAPTER 4).

\section{Long-term physiological and neurological changes after FS}

Neurogenesis is not only important for plasticity of the brain in response to injury, as probably occurs after FS, it has been suggested to also play a role in hippocampal plasticity accompanying processes of learning and memory. However, there is evidence both in favor and against a role for neurogenesis in learning. For example, situations known to cause an increase or decrease in proliferation, are also known to enhance or diminish hippocampal learning, respectively. In contrast, a decreased neurogenesis does not necessarily leads to memory deficits. As contradicting as the evidence of a link between neurogenesis and learning, are the data about long-term cognitive outcome after FS. Some reported a cognitive deficit [14], others a normal development $[15,16]$, and some even a better cognitive performance [17] in children with FS. We found that cognition and behavior was not affected by FS (CHAPTER 6 ). 
Others have shown that HT-induced FS cause hyperexcitability, decreasing the seizure threshold, and eventually result in the development of spontaneous seizures in $35-87 \%$ of the animals starting at 24 or 11 weeks after FS, respectively $[18,20]$. It is plausible that a longer duration of the FS is related to earlier development and a higher prevalence of spontaneous seizures or 'epilepsy' in these studies. We determined the threshold for developing seizures by blocking glutamate transport (CHAPTER 5 ) but found it not to be changed by FS. Furthermore, the EEG measurements in these animals, at 8 and 9 weeks after FS, indicated that at that time point spontaneous seizures were not yet present. This is not surprising, given the fact that the FS in our studies usually do not last longer than about 10 minutes and that longer duration seems to be associated with earlier development of epilepsy. So, the follow-up period may not have been long enough in our study to be able to detect spontaneous seizures.

\section{Methodological issues}

To investigate the link between an early-life event such as FS, and the development and progression of TLE many years later, human studies are not suitable if urgent questions need answering. Animal models can help to overcome this problem. First of all, the life span of a rat is much shorter and its behavior is easy to control and manipulate. Secondly, although human TLE tissue is easily accessible because in many patients suffering from HS the damaged tissue is resected to suppress seizure activity, human control tissue is often post-mortem tissue or tissue resected during brain surgery, thus seldom non-pathological. Not only is animal tissue easier to get hold of, one can sacrifice animals at any time point during the disease process. While in humans tissue is only available at the end-stage of TLE, animal tissue can be obtained during the latent period as well, when no clinical signs of TLE are present and the effects of the FS themselves and the process of epileptogenesis can be studied. However, animal models often focus on a specific aspect of the 
disease, and since TLE has been recognized as a multifactorial disease, may underestimate the link between FS and TLE.

The animal model used in our experiments also has some limitations. One important fact is that we used HT and not fever to induce seizures. However, besides fever, HT by itself can also cause seizures. This occurs for example when children are bathed in hot water. Furthermore, both fever and HT induce cytokine production, thus the immunological influence of FS on the development of TLE is included when studying the effect of HT-induced seizures.

Another limitation of the studies presented in this thesis is that we did not use EEG to confirm the occurrence or absence of FS in our animals during HT treatment. So some animals which we thought did not develop FS, may have had subclinical seizures. Additionally, this means that our HT control group, i.e. rats that did not develop behavioral seizures after HT, is not ideal. However, in clinical studies, one can also not distinguish between the effects of the seizure and the effects of the fever inducing the seizures.

Finally, and most importantly, human and rat brain development are not similar. Human brains, though still maturing after birth, are relatively 'developed' at delivery. In contrast, it takes until the end of the second postnatal week before the rat brain reaches the same maturational state as a human neonate. Therefore, the rats in the animal model used in our experiment have a specific age (10 days old) of which it is known that brain maturation is comparable to that of a child when it is most susceptible for developing seizures. This should improve matching between experimental and clinical data. 


\section{Conclusion: clinical relevance of the findings}

The increased number and the changed phenotype of newborn cells, the presence of mossy fiber sprouting, and the increased T2 signal intensity and metabolic changes found after HT-induced FS, are signs of the development of HS. Other major hallmarks of HS, such as hippocampal atrophy and gliosis however, were not detected. For long it was thought that complex or prolonged FS were particularly associated with the development of HS-associated MTLE. Recently, it became clear that simple FS are also able to cause HS, but strikingly these patients had not developed epilepsy [21]. It is still possible that they will develop epilepsy later on. This is in agreement with the results presented in this thesis. Although signs of HS were found, spontaneous seizures were not (yet) present. FS are still thought by most clinicians to be a benign condition, without an adverse outcome. Although HT-induced FS in this thesis did not cause major physiological, neurological or cognitive problems, subtle and important changes were noticed.

To summarize, the data presented here show that FS can cause long-term changes in the brain, mainly structural. Perhaps these changes, when combined with other risk factors, may worsen the prospect of long-term outcome after FS. 


\section{References}

1. Cendes, F., et al., Early childhood prolonged febrile convulsions, atrophy and sclerosis of mesial structures, and temporal lobe epilepsy: an MRI volumetric study. Neurology, 1993, 43(6), 1083-1087.

2. Falconer, M.A., Serafetinides, E.A., and Corsellis, J.A., Etiology and Pathogenesis of Temporal Lobe Epilepsy. Arch. Neurol., 1964, 10, 233-248.

3. Kuks, J.B., et al., Hippocampal sclerosis in epilepsy and childhood febrile seizures. Lancet, 1993, 342(8884), 1391-1394.

4. Berg, A.T., et al., Childhood-onset epilepsy with and without preceding febrile seizures. Neurology, 1999, 53(8), 1742-1748.

5. Camfield, P., et al., What types of epilepsy are preceded by febrile seizures? A populationbased study of children. Dev. Med. Child Neurol., 1994, 36(10), 887-892.

6. Fernandez, G., et al., Hippocampal malformation as a cause of familial febrile convulsions and subsequent hippocampal sclerosis. Neurology, 1998, 50(4), 909-917.

7. VanLandingham, K.E., et al., Magnetic resonance imaging evidence of hippocampal injury after prolonged focal febrile convulsions. Ann. Neurol., 1998, 43(4), 413-426.

8. Germano, I.M., et al., Neuronal migration disorders increase susceptibility to hyperthermiainduced seizures in developing rats. Epilepsia, 1996, 37(9), 902-910.

9. Scantlebury, M.H., et al., Febrile seizures in the predisposed brain: A new model of temporal lobe epilepsy. Ann. Neurol., 2005, 58(1), 41-49.

10. Forsgren, L., et al., A prospective incidence study of febrile convulsions. Acta Paediatr. Scand., 1990, 79(5), 550-557.

11. Nelson, K.B., Ellenberg, J.H., Prognosis in children with febrile seizures. Pediatrics, 1978, 61(5), 720-727.

12. Berg, A.T., et al., Predictors of recurrent febrile seizures. A prospective cohort study. Arch. Pediatr. Adolesc. Med., 1997, 151(4), 371-378.

13. Dube, C., et al., Serial MRI after experimental febrile seizures: Altered T2 signal without neuronal death. Ann. Neurol., 2004, 56(5), 709-714.

14. Toth, Z., et al., Seizure-induced neuronal injury: vulnerability to febrile seizures in an immature rat model. J. Neurosci., 1998, 18(11), 4285-4294.

15. Manganas, L.N., et al., Magnetic resonance spectroscopy identifies neural progenitor cells in the live human brain. Science, 2007, 318, 980-985

16. Shapiro, L.A., Korn, M.J., Ribak, C.E., Newly generated dentate granule cells from epileptic rats exhibit elongated hilar basal dendrites that align along GFAP-immunolabeled processes. Neuroscience, 2005, 136(3), 823-831.

17. Danbolt, N.C., Glutamate uptake. Prog. Neurobiol., 2001, 65(1), 1-105. 
18. Kwak, S.-E., et al., Hyperthermic seizure induces persistent alteration in excitability of the dentate gyrus in immature rats. Brain Res., 2008, 1216, 1-15.

19. Dube, C., et al., Prolonged febrile seizures in the immature rat model enhance hippocampal excitability long term. Ann. Neurol., 2000, 47(3), 336-344.

20. Dube, C., et al., Temporal lobe epilepsy after experimental prolonged febrile seizures: prospective analysis. Brain, 2006, 129(4), 911-922.

21. Auer, T., et al., History of simple febrile seizures is associated with hippocampal abnormalities in adults. Epilepsia, 2008, 49(9), 1562-1569.. 


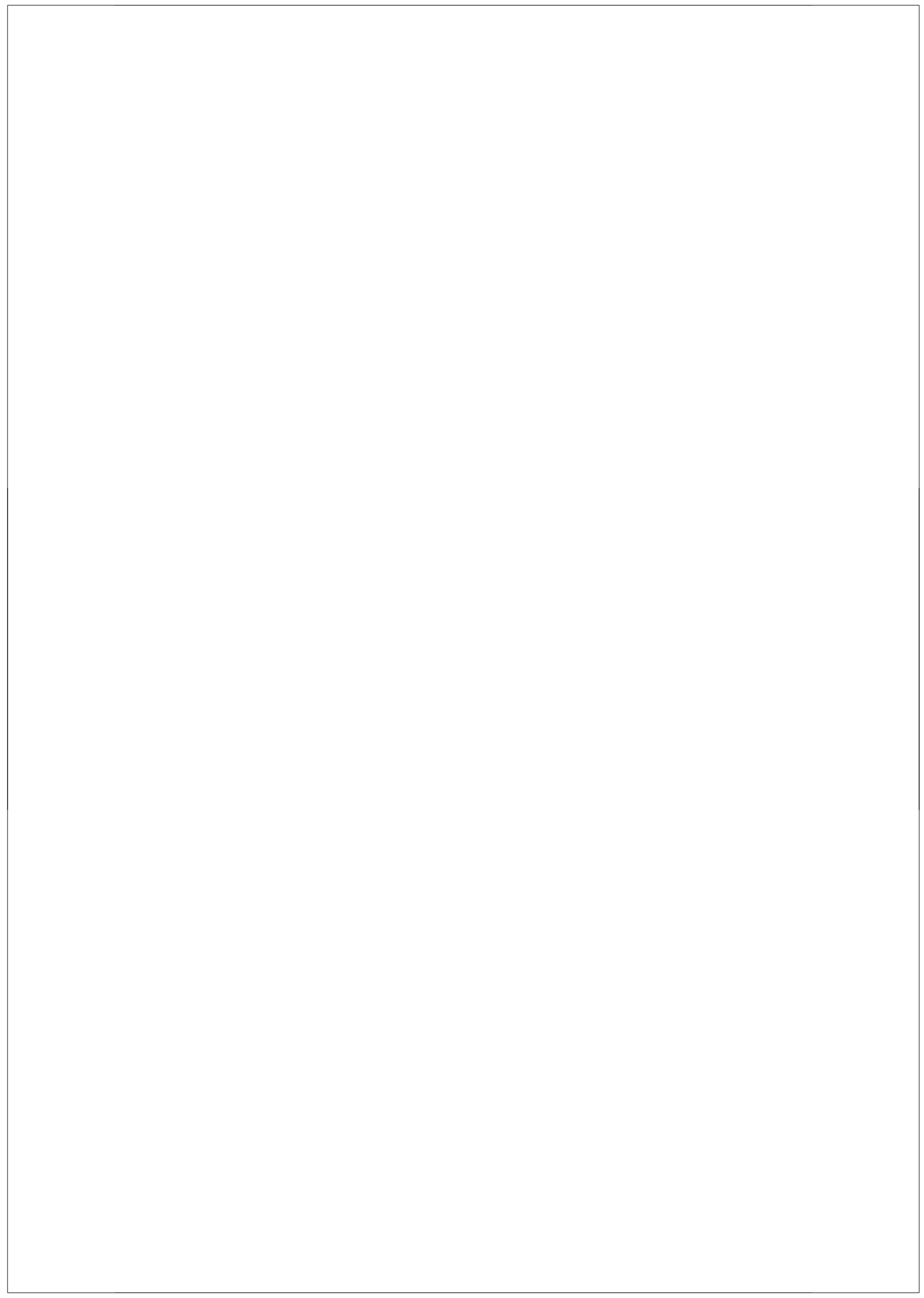




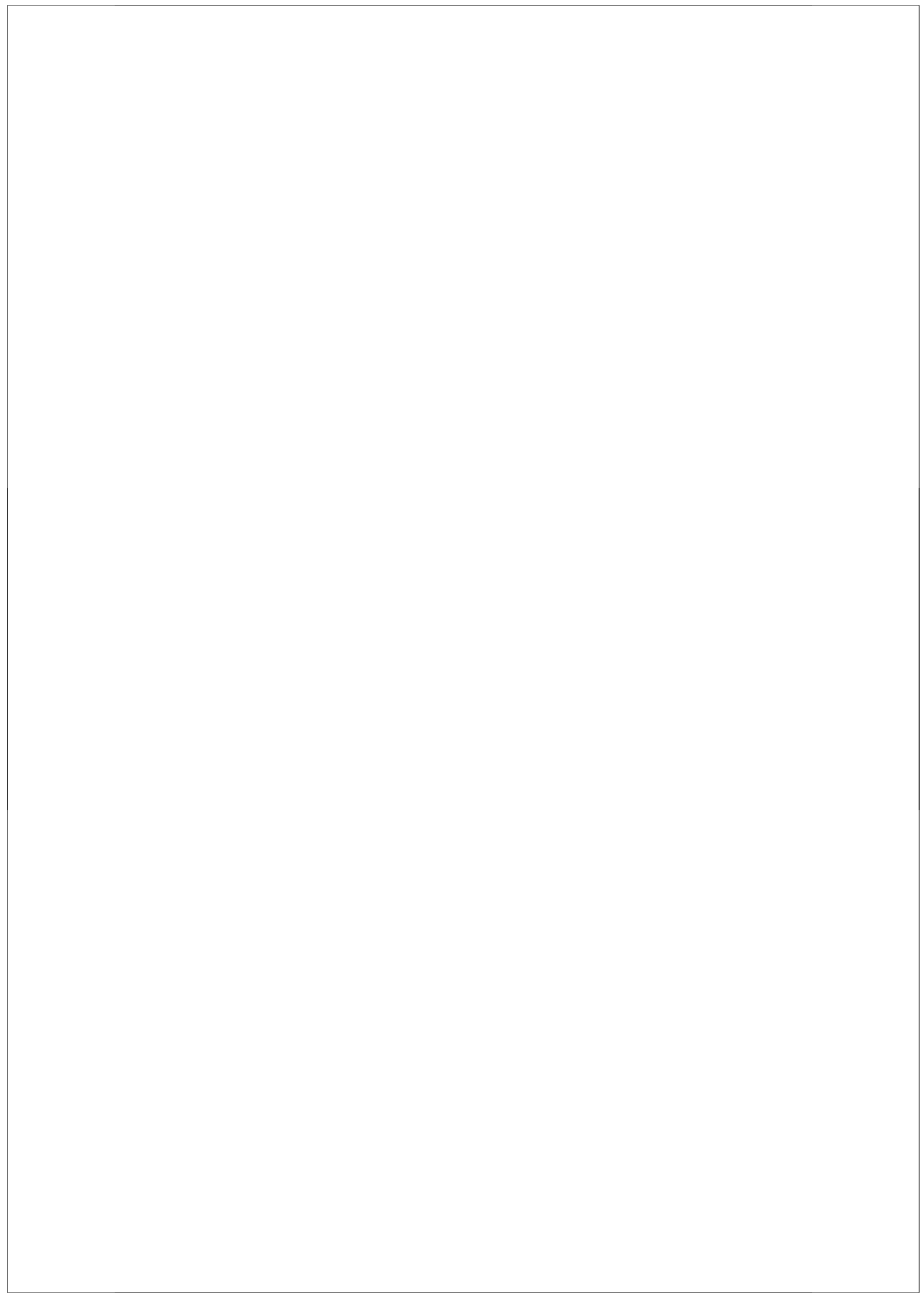




\section{Summary}

The precise nature of the relationship between febrile seizures (FS) and temporal lobe epilepsy (TLE) has been a major controversial issue in epilepsy research for many years. Because FS occur during a critical period of brain development, we investigated possible FS-induced changes in developmental processes, focusing on glutamatergic neurotransmission, and their role in the long-term outcome of FS.

Because FS occur during a critical period of brain development, we focused on possible changes in developmental processes after inducing FS in rats by means of hyperthermia. In CHAPTER 2, we demonstrated that HT-induced seizures, or experimental FS, cause an increase in proliferation and furthermore, an increased survival of newborn cells. In CHAPTER 3, we showed that the 'fate' or phenotype of these newborn cells is altered after experimental FS. Although most of these cells developed into neurons, less expressed the neuronal glutamate transporter, which is important in the process of hyperexcitability. However, when comparing overall expression of all the major glutamate transporters present in the brain, we failed to show a change after FS. It was therefore not surprising that we were unable to induce seizures by blocking glutamate transport (CHAPTER 5). Gliosis was never found. Longterm cognitive and behavioral outcome was also not adversely affected by FS (CHAPTER 6). However, in a prospective study, using different magnetic resonance imaging (MRI) techniques and histology, we did find FS-induced microstructural and metabolic changes (CHAPTER 4). Although these were subtle effects, they might represent an initial sign of the process of epileptogenesis.

Since experimental FS can induce spontaneous seizures, we suggest that hippocampal sclerosis, the pathological substrate often found in TLE patients, especially in those patients that experienced FS during childhood, is a consequence of ongoing seizure activity, not the cause of TLE. 


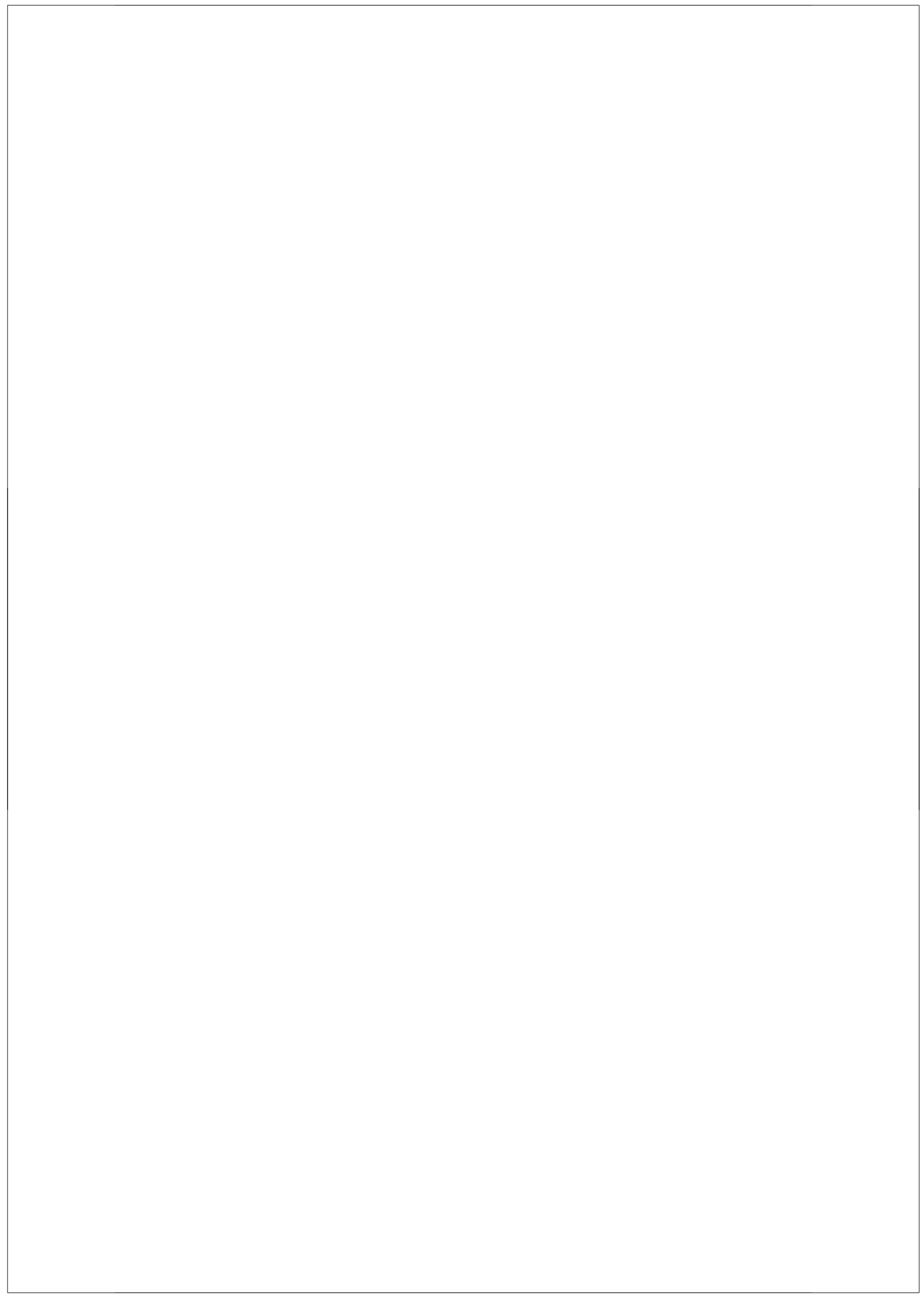




\section{Samenvatting}

Reeds decennia geleden werd vastgesteld dat er een relatie bestaat tussen het doormaken van koortsstuipen in de kindertijd en temporaalkwab epilepsie tijdens de volwassenheid. Nochtans blijt de precieze aard van deze relatie een bron van tegenstrijdigheid in het epilepsieonderzoek. Aangezien koortsstuipen plaatsvinden tijdens een kritische periode in de hersenontwikkeling, werden de mogelijke (door koortsstuipen geïnduceerde) veranderingen op processen, belangrijk tijdens deze ontwikkeling, onderzocht met een focus op glutamaterge neurotransmissie, en de rol hiervan op lange termijn effecten van koortsstuipen.

Hiertoe werden koortsstuipen geïnduceerd bij ratten middels hyperthermie. In HOOFDSTUK 2 werd aangetoond dat deze door hyperthermie geïnduceerde stuipen, ook experimentele koortsstuipen genoemd, een verhoogde proliferatie en overlevingskans van nieuwe cellen teweegbrengen. In HOOFDSTUK 3 staat beschreven dat de 'bestemming' of het fenotype van deze nieuwe cellen veranderd is na koortsstuipen; ondanks dat ze meestal uitgroeien tot neuronen, brengen minder van deze cellen de neuronale glutamaattransporter tot expressie, wat belangrijk kan zijn bij het proces van hyperexciteerbaarheid. Maar, de globale expressie van alle types glutamaattransporters in de hersenen bleek niet veranderd na koortsstuipen. Bijgevolg is het niet verrassend dat blokkering van het glutamaattransport geen epileptische aanval tot gevolg had (HOOFDSTUK 5). Gliose werd nooit gevonden. Lange termijn effecten op cognitie en gedrag waren nagenoeg afwezig (HOOFDSTUK 6). In een prospectieve studie echter, waar gebruik werd gemaakt van MRI gecombineerd met histologie, werden microstructurele and metabolische veranderingen gedetecteerd (HOOFDSTUK 4). Hoewel slechts subtiel, zouden deze effecten een initieel signaal kunnen voorstellen van epileptogenese.

Daar gekend is dat experimentele koortsstuipen epileptische aanvallen kunnen veroorzaken, suggereren de data hier beschreven dat hippocampus sclerose (hét pathologisch substraat gevonden bij de meeste temporaalkwab epilepsie patiënten, vooral bij dezen die eveneens koortsstuipen hebben doorgemaakt), een gevolg zijn van aanhoudende aanvalsactiviteit, en niet de oorzaak. 


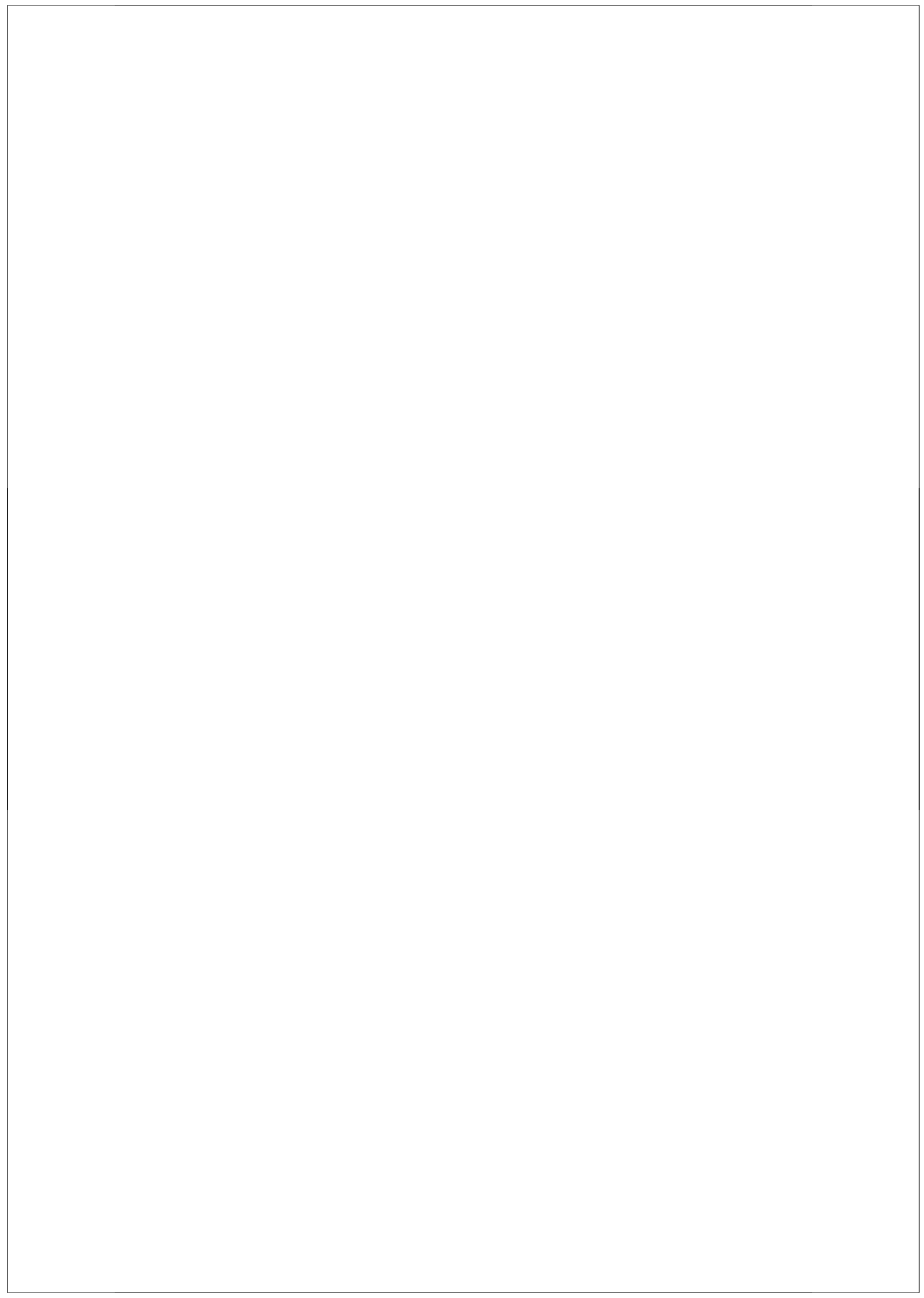




\section{Dankwoord}

Het afronden van een promotieonderzoek gaat met vallen en opstaan. En in het dankwoord dat hoort bij dit proefschrift (hetgeen meestal wél gelezen wordt, in tegenstelling tot de rest van de inhoud (:) zou ik iedereen willen bedanken die, op welke manier dan ook, heeft bijgedragen tot de totstandkoming. Er zijn natuurlijk enkele personen die ik in het bijzonder wil bedanken.

Beste Prof. Beuls, u hebt van in het begin in mij geloofd; vooral dankzij u heb ik dit onderzoek kunnen doen. Als u er niet geweest was, had ik waarschijnlijk nooit Marie Curie Fellow kunnen worden. Bij u kon ik steeds terecht met vragen die niet gericht waren op de inhoud van het onderzoek, want daarvoor was Govert er. Bedankt voor de kansen en de steun die u mij heeft gegeven en ik wens u nog veel succes met uw nieuwe 'carrière'!

Prof. Steinbusch, beste Harry, de eerste jaren hebben we elkaar eigenlijk niet zoveel gesproken. Maar dat heeft mij de vrijheid gegeven om zo zelfstandig mogelijk te kunnen werken. Pas toen Prof. Beuls met pensioen ging, en er voor mijn promotie nog een lange weg moest worden afgelegd, bent $u$ een belangrijkere rol gaan spelen. Ik wil u dan ook vooral bedanken voor uw hulp bij het afronden van mijn promotie.

Govert, nu is het dan zover, mijn tijd in Maastricht zit er op. Toen ik een zestal jaar geleden samen met Brenda mijn eerste stappen zette in het onderzoek, keek ik met verwondering naar de AIO's van toen. Omdat ik nooit had gedacht dat ik één van hen zou worden, kwam het dan ook een beetje als een verrassing toen je zei dat ik, indien ik dat graag wilde, nog een tijdje kon doorgaan met de koortsstuipen. En voor ik het helemaal besefte, was ik Marie Curie Fellow geworden. Helaas werd het oorspronkelijke project (remember, de 'FS muizen') niet gehonoreerd en moesten we met weinig middelen verder. Desondanks hebben we toch iets moois bereikt. Zelfs Prof. Baram is onder de indruk, ondanks dat we het niet altijd met haar eens zijn. Jij was diegene die het 
steeds 'positief' zag. Een voorbeeld: als we samen achter de microscoop zaten, was jij ervan overtuigd specifieke kleuring te zien, terwijl ik het sterk op 'achtergrond' vond lijken. Ik bleef liever met beide voeten op de grond, wilde alles dubbel checken om zeker te zijn. Een promotieonderzoek is een leerproces, niet alleen voor de AIO, maar ook voor de begeleider, zeker als hij voor het eerst de taak van copromotor op zich neemt. Discussiëren konden we wel, maar ik ben van mening dat de komst van Kim ons beiden veel deugd heeft gedaan, want de derde persoon kan de doorslag geven bij een beslissing. Om af te sluiten, zou ik het volgende willen zeggen: ik heb veel geleerd uit dit avontuur, en ik hoop jij ook. Dat het jou mag helpen om nog lang door te gaan met het epilepsieonderzoek. Bedankt!

Liefste Kim, het zal voor velen geen verrassing zijn dat ik jou koos als één van mijn paranimfen want we hebben samen heelwat beleefd op het lab en daarbuiten. Toen jij het bureau van Roselie kwam innemen, had ik het eerst wat moeilijk, maar lang heeft dat niet geduurd, want al gauw bleek dat we elkaar veel konden leren. Jij leerde mij elektroden implanteren, en ik wist altijd (lees 'meestal') wel raad met de problemen van het bejaarde EEG systeem. Bedankt voor alles en veel succes met het afronden van jouw promotie en vooral met 'mama zijn'; dat er nog vele Woutertjes mogen volgen :)

Liefste Imke, tot voor kort behoorden wij tot de garde van de 'oude AIO's' en dat schept toch wel een band. We hebben de voorbije jaren lief en leed gedeeld in het lab, en daarom koos ik jou als mijn paranimf. We deelden ook de mening dat elders ideeën opdoen wel eens goed kan zijn om te 'groeien'. Jij bent zo terecht gekomen bij Roche, ik bij de Universiteit Hasselt. Ik wens je veel succes met jouw nieuwe baan en met alles wat je nog wil bereiken in dit leven! Spijtig dat je bij de promotie zelf niet aanwezig kunt zijn, maar er is voor prima vervanging gezorgd. Liefste Rinske, bedankt dat jij mij wou bijstaan als derde paranimf! 
Ik zou bij deze ook graag alle collega's bedanken van de afdeling Neuroscience van de School of Mental Health and Neuroscience, zoals dat tegenwoordig heet. Al van de eerste minuut heb ik mij daar thuis gevoeld, en dat dankzij jullie. In het bijzonder de 'meisies', Marijke, Eveline, Annerieke, Rinske, Kim, en Imke. Een perfecte combinatie van zwart-geel-rood en blauw-wit-rood (:) Ik zal jullie heel erg missen. En als jullie ooit mijn hulp willen inroepen, voor eender welk probleem, vergeet dan niet: "Mega-Evi staat paraat!". Overall, I would like to wish all the $\mathrm{PhD}$ students good luck with finishing their PhD. Ook bedankt Hellen en Marjanne voor de hulp in het lab, de secretaresses Akke, Mirèse, Sandra, en Marie-Thérèse voor het regelen van zoveel administratieve rompslomp die bij een promotie hoort, en Nicole en Anja, voor jullie eindeloos enthousiasme voor EURON!

Hetzelfde geldt voor de collega's van de neurochirurgie. Hen zou ik voornamelijk willen bedanken om collectief aanwezig te zijn als het mijn beurt was om iets te vertellen voor de wetenschapsvergadering op dinsdagavond. Beste Olaf, ik wens je nog veel succes met jouw epilepsieonderzoek.

De medewerkers van het CPV (Centrale Proefdier Voorzieningen), in het bijzonder Richard, Allard, en Harry, wil ik eveneens bedanken. Jullie snelden meteen ter hulp als ik weer eens aan de telefoon hing met een dringend verzoek. En de professionaliteit waarmee jullie met de dieren omgaan, is echt bewonderenswaardig. Aan de studenten die ik heb mogen begeleiden, Margreet, Xander, Benjamin, en Julia, zou ik een belofte willen doen. Ik weet, het uitgevoerde onderzoek naar de invloed van polymorfismen bij koortsstuipen en epilepsie, heeft tot nu toe nog niet geleid tot een publicatie, maar reken erop dat we de moed niet opgeven tot dat artikel er is.

Beste Jaap en Walter, het was fijn samenwerken, dus bedankt! Het heeft lang geduurd voor ons artikel werd gepubliceerd, maar de aanhouder wint, en ik denk dat jullie het wel met mij eens zijn als ik zeg dat we er trots op mogen zijn. Ook bedankt Vivianne, Paul, en Erwin. Zonder jullie hulp hadden we het EEG systeem nooit aan de praat gekregen én draaiende kunnen houden. 
Roselie, je dacht misschien dat ik je zou vergeten in dit dankwoord, maar niets daarvan. Ach, wat hebben we toch veel gelachen samen. Vooral onze buitenlandse kamergenotes Kasia en Chunyan hebben ons vaak doen huilen van plezier. De ene omdat ze na een trip naar Polen tijdens de paasvakantie plots getrouwd én met een kinderwens terug naar Nederland was gekomen; de ander omdat ze ongebakken broodjes at, en ze onze gesprekken opnam waardoor ze al gauw tot 'Chinese Spy' werd gekroond. Ik zal nooit vergeten hoe de laptop van Chunyan ons (en Wiel ()) vaak tot waanzin dreef als die weer eens alles van het Engels naar het Chinees ging vertalen en omgekeerd, LUIDOP! We hebben samen een leuke tijd gehad, en ondanks dat we elkaar niet zo vaak meer zullen zien, hoop ik dat we voor altijd vriendinnen mogen blijven. Veel succes met jouw carrière als oogarts, je gaat het fantastisch goed doen!

Sinds enkele maanden ben ik weer terug van weggeweest bij de Universiteit Hasselt. Na mijn studies Biomedische Wetenschappen had ik zin in iets anders en ben ik naar Maastricht getrokken. Maar er is een spreuk die luidt 'home sweet home'. En jullie, collega's bij BIOMED, hebben mij een nieuwe 'thuis' gegeven. Bedankt Piet, Niels en Jerome om mij die kans te geven. Beste Katrien, ik hoop dat we nog lang samen mogen werken, want ik mij amuseer mij ontzettend met jou in het labo.

Make en pake, jullie hebben mij destijds de kansen en de vrijheid gegeven om zelf uit te zoeken wat en waar ik graag wilde gaan studeren. Daar ben ik jullie dankbaar voor. Jullie hebben mij altijd veel liefde en steun gegeven. Ik ben trots op jullie en hoop stiekem dat jullie ook trots zijn op mij.

Liefste Jorge, liefste loekie, zonder jou zou ik nooit zo gelukkig zijn geweest. En nu mijn doctoraat achter de rug is, kan ik niet wachten om het huisje van onze dromen te gaan bouwen. Ik kijk er echt naar uit. Ik zie je graag! 


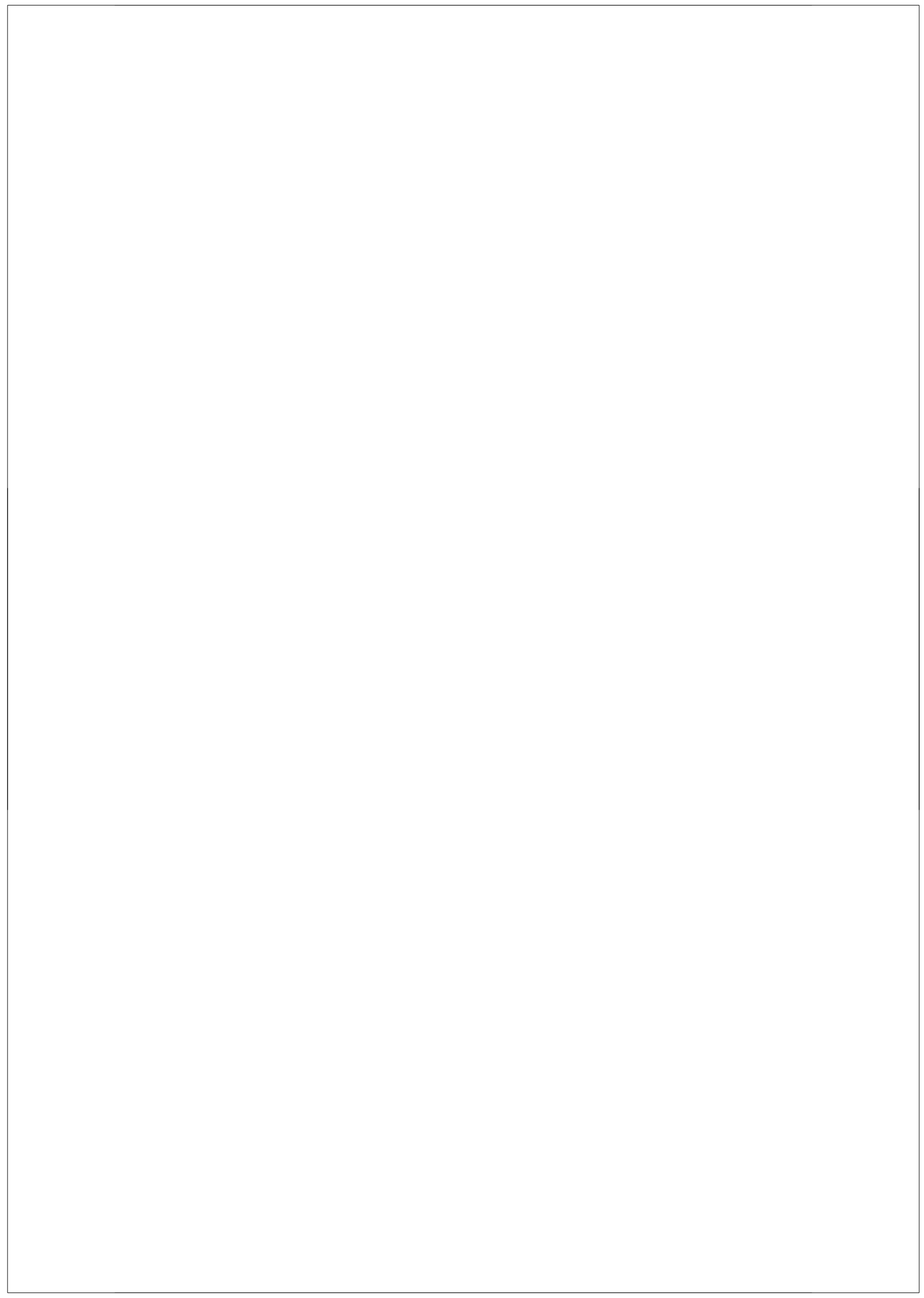




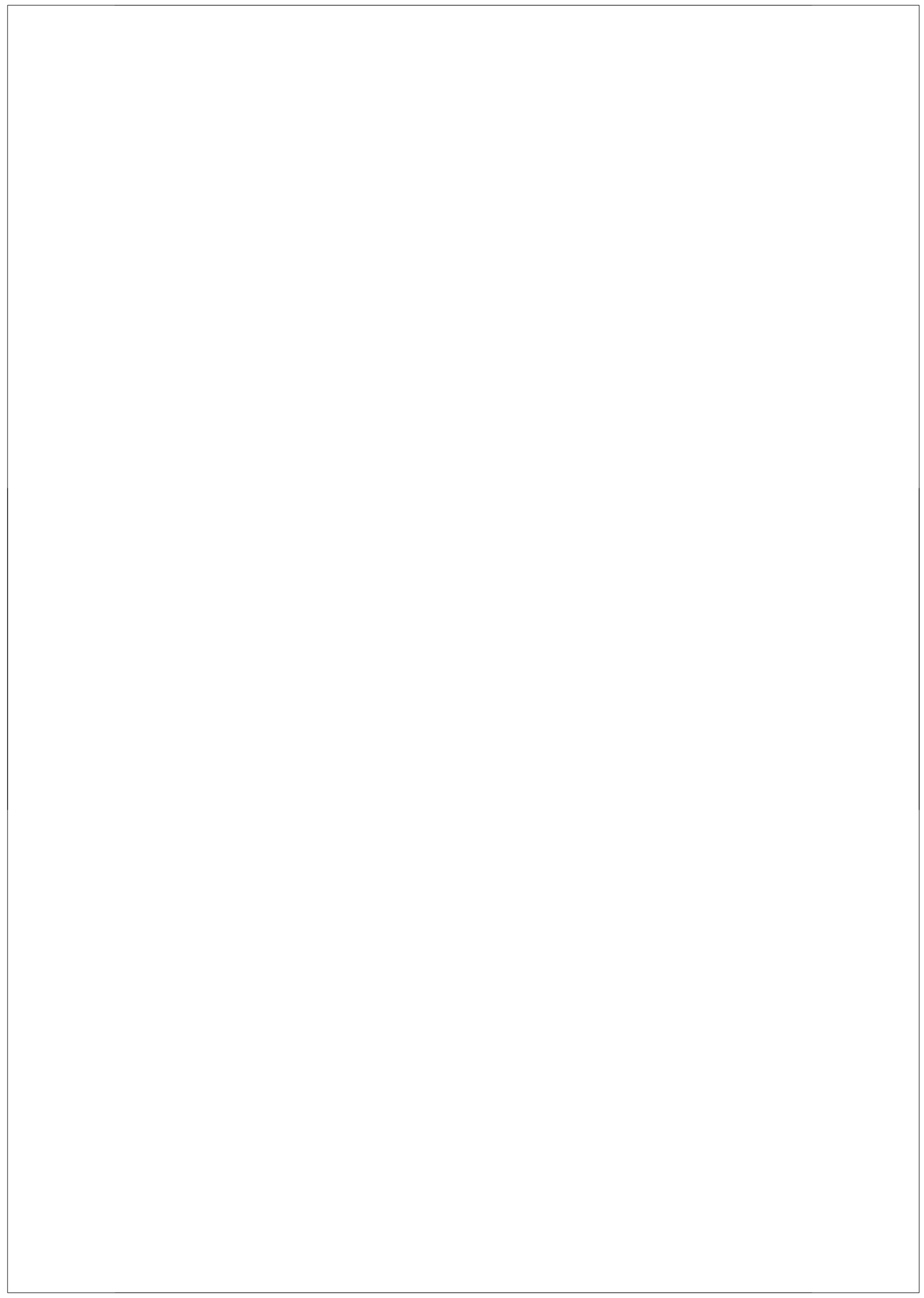




\section{List of publications}

\section{Original papers}

Lemmens, E.M. ${ }^{*}$, Aendekerk, B. ${ }^{*}$ Schijns, O.E., Blokland, A., Beuls, E.A., Hoogland, G., Long-term behavioral outcome after early-life hyperthermiainduced febrile seizures? (under review 'Epilepsy and Behavior'; *Authors contributed equally).

Jansen, J.F.*, Lemmens, E.M. ${ }^{*}$, Strijkers, G.J., Prompers, J.J., Schijns, O.E., Kooi, M.E., Beuls, E.A., Nicolay, K., Backes, W., Hoogland, G., Short- and longterm limbic abnormalities after experimental febrile seizures. Neurobiology of Disease, 2008, July 27 (online early; *Authors contributed equally).

Schijns, O.E., Visser-Vandewalle, V., Lemmens, E.M., Janssen, A., Hoogland, G., Surgery for temporal lobe epilepsy after cerebral malaria. Seizure, 2008, May 30 (online early).

Lemmens, E.M., Schijns, O.E., Beuls, E.A., Hoogland, G., Cytogenesis in the dentate gyrus after neonatal hyperthermia-induced seizures: What becomes of surviving cells? Epilepsia, 2008, 49(5), 853-860.

Lemmens, E.M., Lubbers, T., Schijns, O.E., Beuls, E.A., Hoogland G., Gender differences in febrile seizure-induced proliferation and survival in the rat dentate gyrus. Epilepsia, 2005, 46(10), 1603-1612.

\section{Abstracts}

Schijns, O.E., Karaca, Ü., Lemmens, E.M., Pannek, H.W., Ebner, A., van Kroonenburgh, M.J., Meana, J.J., Callado, L.F., Herscheid, J.D., Hoogland, G., Hippocampal neuronal GABA transporter expression in patients with mesial temporal sclerosis-associated temporal lobe epilepsy. $\mathbf{8}^{\text {th }}$ European Congress on Epileptology, Berlin (Germany), September 2008. 
Swijsen, A., Hoogland, G., Schijns, O.E., Lemmens, E.M., Rigo, J.-M., Characterization of ligand-gated ion channels in newborn dentate granule cells after neonatal hyperthermia-induced seizures. 6th FENS Forum of European Neuroscience, Geneva (Switzerland), July 2008.

Schijns, O.E., Celis, M., Lemmens, E.M., Pannek, H.W., Lahl, R., Ebner, A., Dings, J., van Kroonenburgh, M.J., Herscheid, J.D., Hoogland, G., Hippocampal GABA transporter immunoreactivity in surgically treated patients with temporal lobe epilepsy. $13^{\text {th }}$ European Congress of Neurosurgery (EANS), Glasgow (UK), September 2007.

Lemmens, E.M., Do experimental febrile seizures cause long-term changes in hippocampal MRI and/or ${ }^{1} \mathrm{H}-\mathrm{MRS}$ signals? $6^{\text {th }}$ Endo-Neuro-Psycho Meeting, Doorwerth (The Netherlands), June 2007 (oral presentation).

Jansen, J.F., Lemmens, E.M., Hoogland, G., Schijns, O.E., Jennekens, W., Strijkers, G.J., Prompers, J.J., Habets, J., Kooi, M.E., Nicolay, K., Backes, W., Prospective brain T2 relaxometry, diffusion tensor imaging and spectroscopy in a rat model of early-life febrile convulsions. $27^{\text {th }}$ International Epilepsy Congress, Singapore (Singapore), July 2007 (published in Epilepsia 2007, 48(Suppl. 7), 47).

Lemmens, E.M., Lubbers, T., Schijns, O.E., Beuls, E.A., Hoogland, G., Fate of dentate granule cells born after experimental neonatal seizures. $\mathbf{1}^{\text {st }}$ North American Regional Epilepsy Congress (American Epilepsy Society), San Diego (USA), December 2006 (published in Epilepsia 2006, 47(Suppl. 4), 22; poster presentation).

Lemmens, E.M., Fate of newborn dentate granule cells after neonatal seizures. $9^{\text {th }}$ EURON PhD days, Liège (Belgium), September 2005 (granted with an award for 'best oral presentation').

Hoogland, G., Aendekerk, B., Lemmens, E.M., Visser-Vandewalle, V., de Graan, P.N., Beuls, E.A., Early-life hyperthermia affects locomotor activity in adulthood. Dutch League Against Epilepsy meeting, 'sectie wetenschappelijk onderzoek (SWO)', Utrecht (The Netherlands), December 2003. 


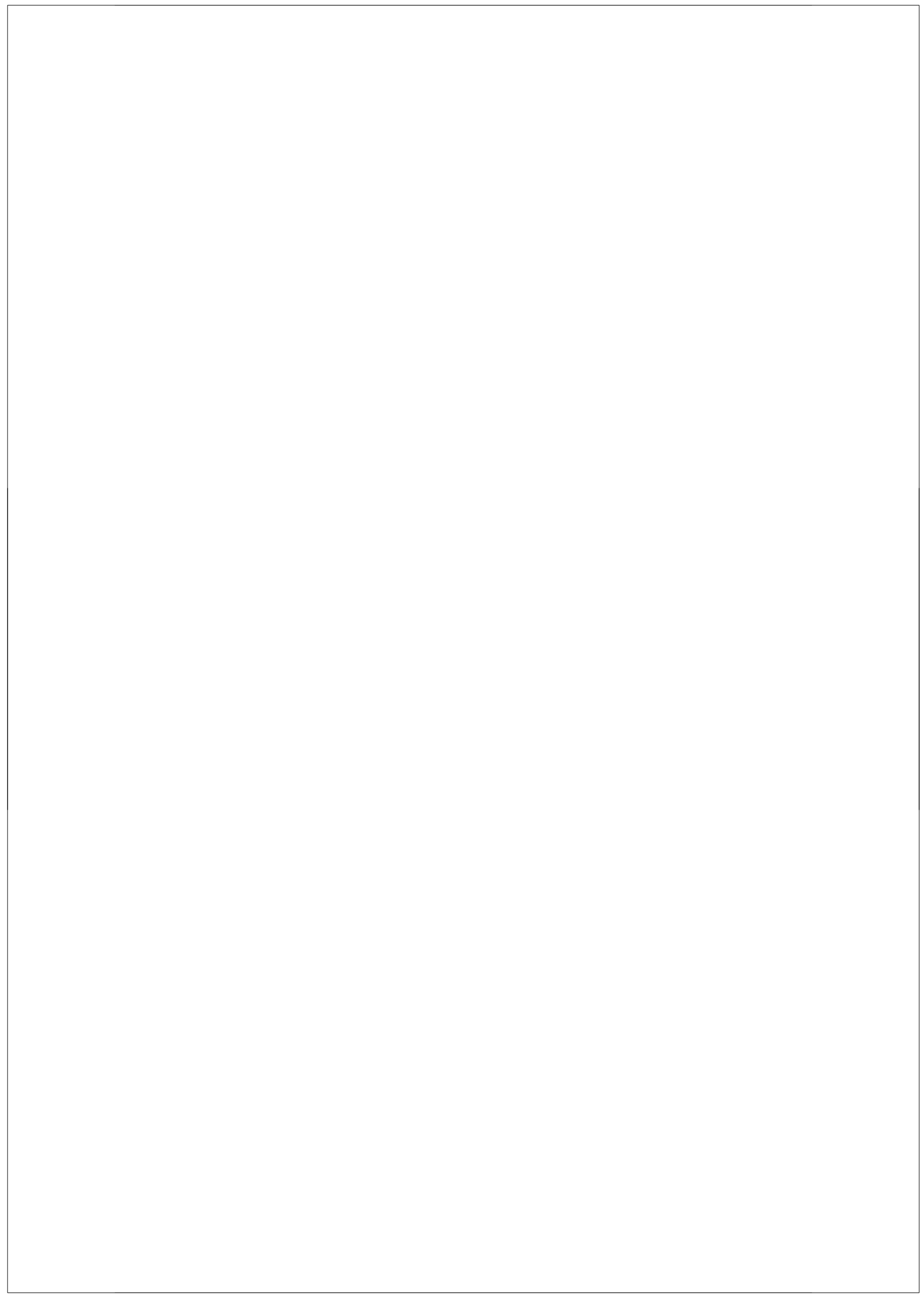




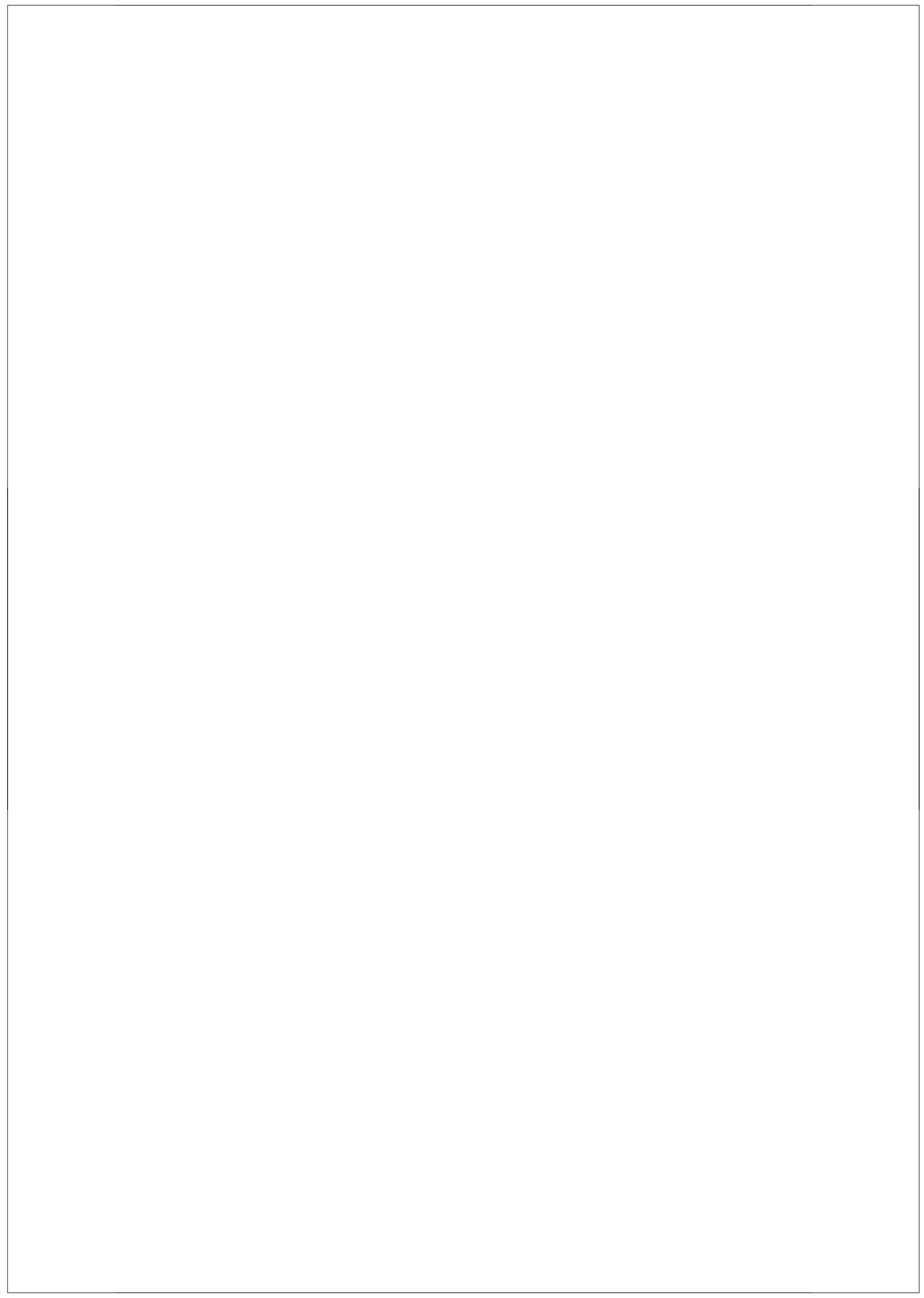




\section{Curriculum Vitae}

Evi Lemmens was born on the $12^{\text {th }}$ of February 1981 in Hasselt, Belgium. After completing her secondary education at the Virga Jesse College in Hasselt in 1999, she started her studies of Biomedical Sciences at the former Limburgs University Center in Diepenbeek, Belgium (now Hasselt University). Two years later, the Transnational University Limburg was founded, a collaboration between Hasselt University in Belgium and Maastricht University in The Netherlands, which made it possible for her to finish her studies in Limburg. The project that started off as a 6-month internship to obtain the degree of Master of Science in 2003, continued as a PhD research project at the Institute of Brain and Behaviour (European Graduate School of Neuroscience, Maastricht University) under the daily supervision of Dr. Govert Hoogland, Prof. Dr. Harry W.M. Steinbusch, and Prof. Emile A.M. Beuls. The PhD project aimed at studying the effects of febrile seizures on the developing brain, and the results are presented in this thesis. Since May 2008 she is working at the Biomedical Research Institute at Hasselt University, Belgium.

Evi Lemmens werd geboren op 12 februari 1981 te Hasselt, België. Na het voltooien van het secundair onderwijs aan het Virga Jesse College te Hasselt in 1999, begon zij haar studies Biomedische Wetenschappen aan het toenmalige Limburgs Universitair Centrum te Diepenbeek, België (nu Universiteit Hasselt). Twee jaar later werd de Transnationale Universiteit Limburg opgericht, een samenwerking tussen de Universiteit Hasselt in België en de Universiteit Maastricht in Nederland, waardoor zij haar studies in Limburg kon voltooien. Het project dat begon als een 6-maand durende afstudeerstage voor het behalen van de graad Master of Science in 2003, werd verder gezet als een PhD project bij het instituut Hersenen en Gedrag (European Graduate School of Neuroscience, Universiteit Maastricht) onder de dagelijkse begeleiding van Dr. Govert Hoogland, Prof. Dr. Harry W.M. Steinbusch en Prof. Emile A.M. Beuls. Het onderzoek had als doel het bestuderen van de effecten van koortsstuipen op het ontwikkelende brein, en de resultaten zijn beschreven in dit proefschrift. Sinds mei 2008 is zij werkzaam bij het Biomedisch Onderzoeksinstituut van de Universiteit Hasselt, België. 


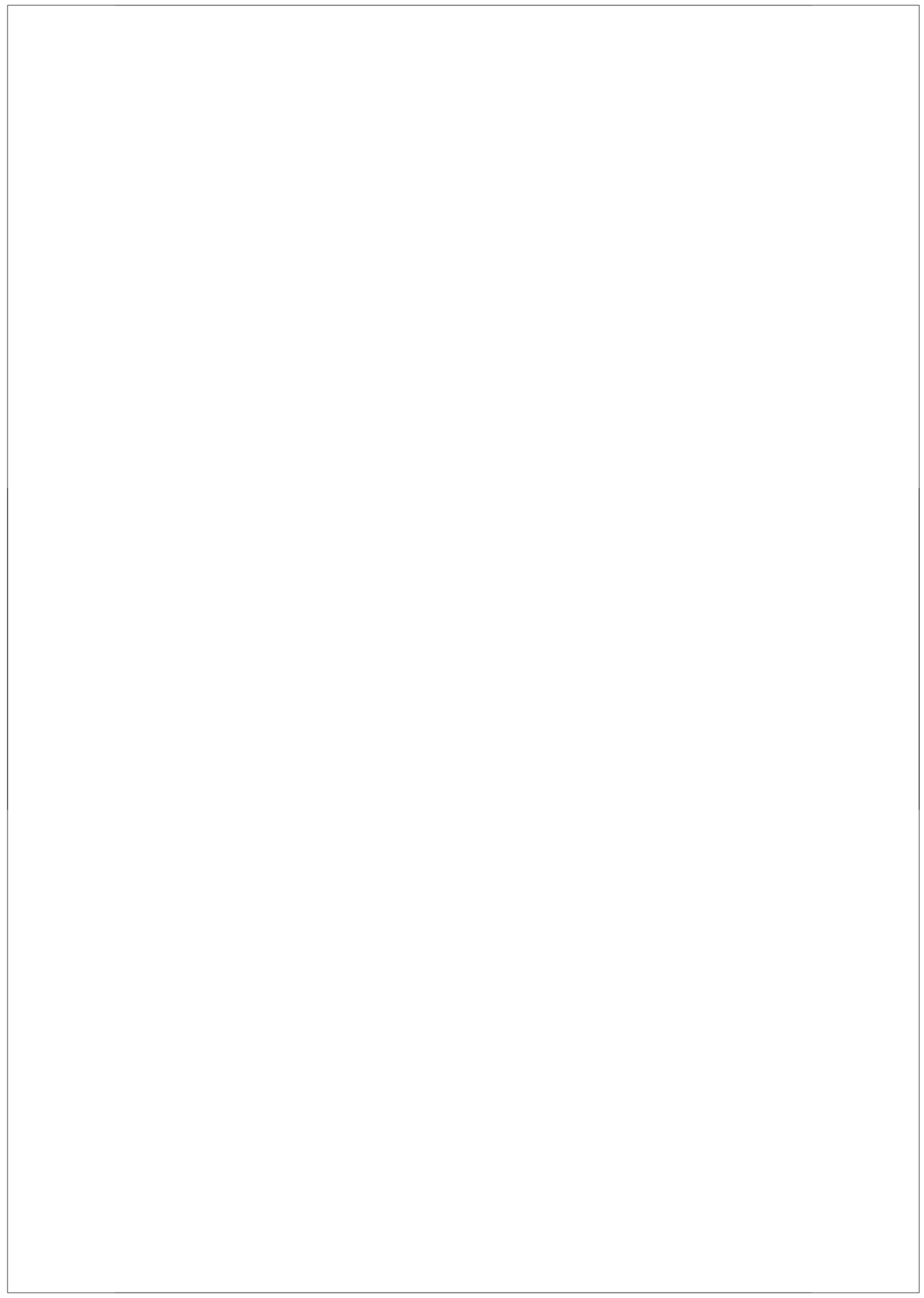




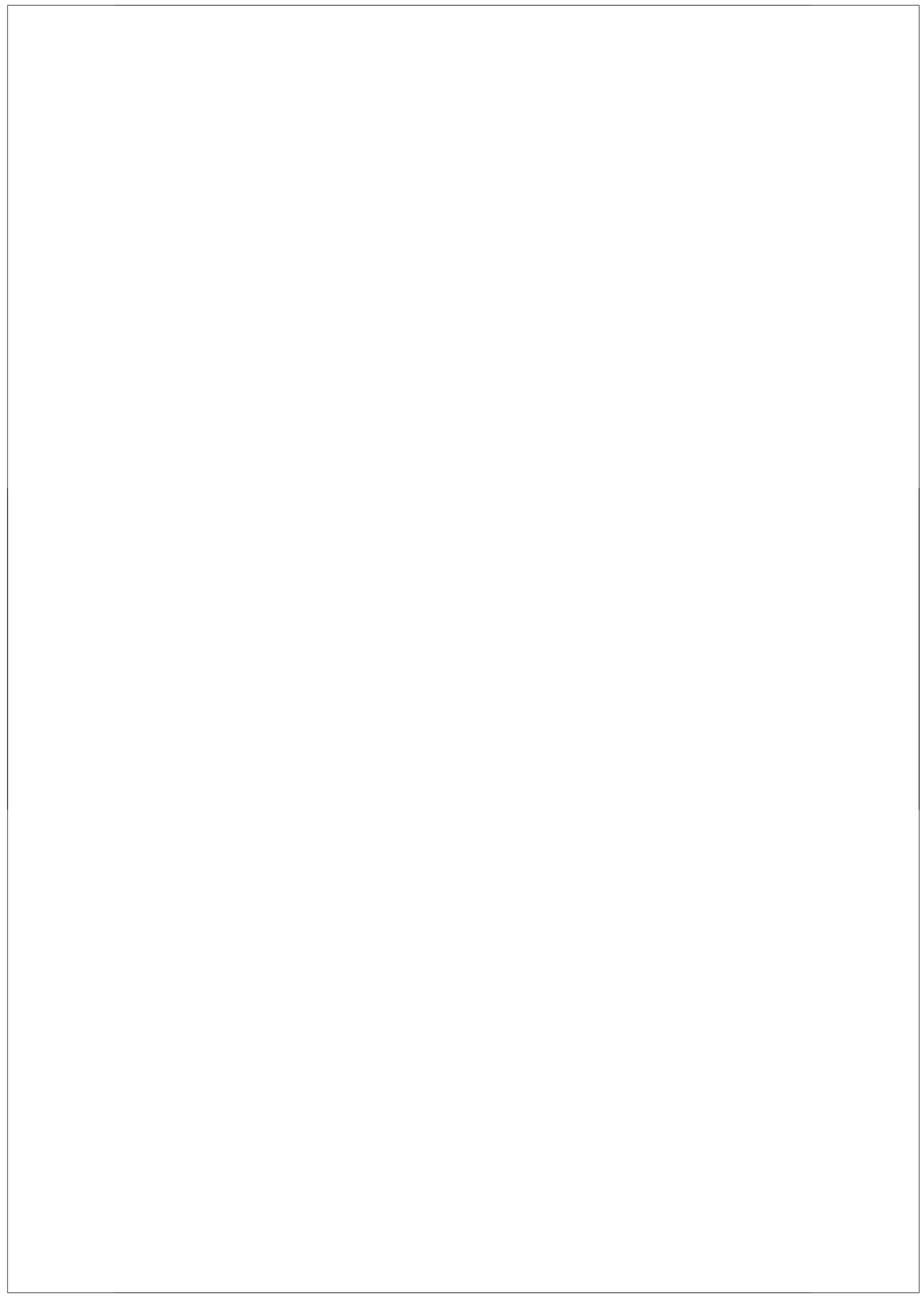




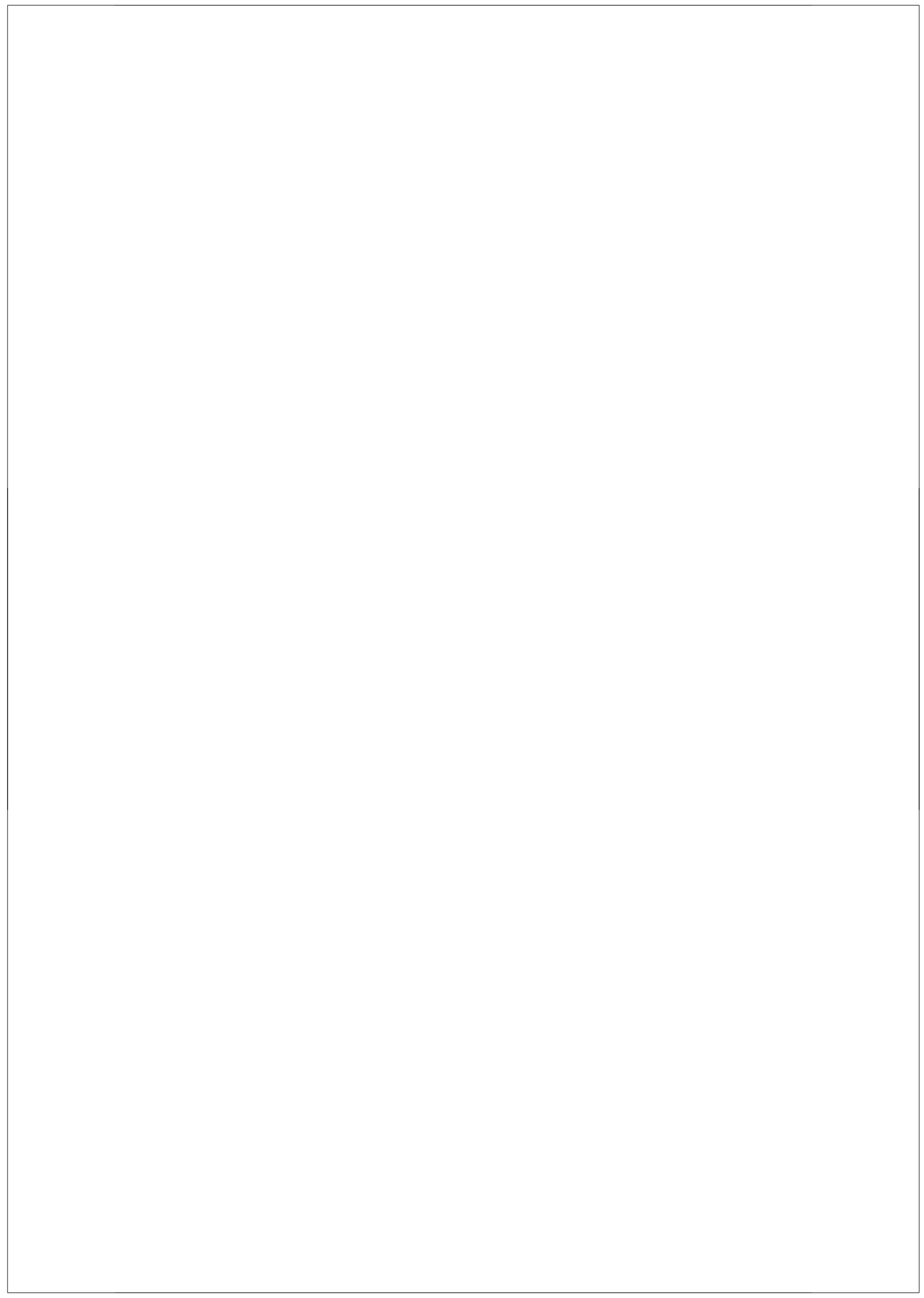

